\title{
The New Minimal Supersymmetric GUT : Spectra, RG analysis and Fermion Fits
}

\author{
Charanjit S. Aulakh ${ }^{a}$ and Sumit K. Garg ${ }^{b}$ \\ ${ }^{a}$ Dept. of Physics, Panjab University, Chandigarh, India \\ ${ }^{b}$ Center for High Energy Physics, IISc, Bangalore, India
}

The supersymmetric SO(10) GUT based on the $\mathbf{2 1 0} \oplus \mathbf{1 0} \oplus \mathbf{1 2 0} \oplus \mathbf{1 2 6} \oplus \overline{\mathbf{1 2 6}}$ Higgs system provides a minimal framework for the emergence of the R-parity exact MSSM at low energies and a viable supersymmetric seesaw explanation for the observed neutrino masses and mixing angles. We present formulae for MSSM decomposition of the superpotential invariants, tree level light charged fermion effective Yukawa couplings, Weinberg neutrino mass generation operator, and the $d=5, \Delta B=\Delta L \neq 0$ effective superpotential in terms of GUT parameters. We use them to determine fits of the 18 available fermion mass-mixing data in terms of the superpotential parameters of the NMSGUT and SUGRY(NUHM) type soft supersymmetry breaking parameters $\left(\left\{m_{\tilde{f}}, m_{1 / 2}, A_{0}, M_{H, \bar{H}}^{2}\right\}\right)$ specified at the MSSM one loop unification scale $M_{X}^{0}=10^{16.33} \mathrm{GeV}$. Our fits are compatible with electroweak symmetry breaking and Unification constraints and yield right-handed neutrino masses in the leptogenesis relevant range : $10^{8}-10^{13} \mathrm{GeV}$. Matching the SM data requires lowering the strange and down quark Yukawas in the MSSM via large $\tan \beta$ driven threshold corrections and characteristic soft Susy breaking spectra. The Susy spectra have light pure Bino LSP, heavy exotic Higgs(inos) and large $\mu, A_{0}, M_{H, \bar{H}}$ parameters $\sim 100 \mathrm{TeV}$. Typically third generation sfermions are much heavier than the first two generations. The smuon is often the lightest charged sfermion thus offering a Bino-CDM co-annihilation channel. The parameter sets obtained are used to calculate $\mathrm{B}$ violation rates which are found to be generically much faster $\left(\sim 10^{-28} \mathrm{yr}^{-1}\right)$ than the current experimental limits. Improvements which may allow acceptable B violation rates are identified. 


\section{Introduction}

The discovery of neutrino mass was both preceded by [1] and itself provoked [2, 4, 6, 8] intensive investigation of unifying models that naturally incorporate supersymmetry and the seesaw mechanisms [9] : in particular models with the left-right gauge group as a part of the gauge symmetry and $B-L$ broken at a high scale and R/M-parity preserved to low energies [1]. The close contiguity of the seesaw scale and the Grand Unified scale pushed $\mathrm{SO}(10)$ GUTs, which are the natural GUT home of both Type I and Type II seesaw mechanisms, but were long relegated as baroque cousins of the -seemingly- more elegant minimal SU(5) GUT, into centre stage. The understanding that the Susy $\mathrm{SO}(10)$ GUT based on the $\mathbf{2 1 0} \oplus \mathbf{1 0} \oplus \mathbf{1 2 6} \oplus \overline{\mathbf{1 2 6}}$ Higgs system proposed [10,11] long ago was an optimal candidate for the Minimal Supersymmetric GUT (MSGUT) crystallized after the demonstration of its minimality on parameter counting grounds and an elegant reduction of its spontaneous symmetry breaking problem to a single cubic equation with just one unknown parameter [12. Careful computations of the symmetry breaking [10 12] and mass spectra 3, 4,6,7, became available for the MSGUT. These theories naturally maintain a structural distinction between Higgs and matter fields and therefore naturally preserve R-parity down to low energies [1,2,13.

The initial euphoria $[8$ that the version utilizing only 10, $\overline{\mathbf{1 2 6}}$ Higgs representations might prove sufficient [14 to fit all low energy fermion data in an elegant and predictive way ran aground when the successful generic fits of fermion mass data were shown to be unrealizable [15 17] in the context of the actual seesaw mechanisms(both Type I and Type II) available in the MSGUT. Both types of seesaw yielded neutrino masses that were too small and Type I was shown to generically dominate Type II. Faced with this impasse it is natural to have recourse to the third allowed type of Fermion Mass (FM) Higgs, i.e the 120-plet of SO(10). The 120-plet had previously played a relatively minor role in fitting the fermion mass data [18,19].

In view of our no-go result in the MSGUT, however, we proposed [16] a re-allocation of roles among the three types of FM Higgs representation by suppressing the $\overline{\mathbf{1 2 6}}$ Yukawa couplings relative to those of $\mathbf{1 0 , 1 2 0}$. Since the Type I seesaw neutrino masses are inversely proportional to the $\overline{\mathbf{1 2 6}}$ Yukawa coupling this would enhance the Type I seesaw masses to viable levels(Type II contributions get further suppressed) while perhaps still allowing sufficient freedom to fit all the fermion mass and mixing data. This also has the interesting consequence that right-handed neutrino masses would be significantly lowered into a range $\left(10^{8}-10^{13}\right) \mathrm{GeV}$ compatible with leptogenesis.

Related subsequent work 20 22 gave mixed signals regarding the viability of our proposal to use $\mathbf{1 0}+\mathbf{1 2 0}$ Higgs to fit charged fermion masses while small $\overline{\mathbf{1 2 6}}$ Yukawa couplings to raise Type I neutrino masses by lowering $\overline{\mathbf{1 2 6}}$ vev generated right-handed neutrino masses. However we find accurate NMSGUT specific fits using ultra small $\overline{\mathbf{1 2 6}}$ couplings but a somewhat enlarged fitting scenario that takes recourse to the strong influence (at the large $\tan \beta \sim 50$ values typically favored by SO(10) Susy GUTs) of supersymmetric particle threshold corrections on the down type quark masses. This is done in order to lower the Yukawa couplings of the down and strange quarks to values that can be accommodated by the NMSGUT specific fitting formulae. These fits are manifestly distinct from the accurate generic fits found in [21,22, which besides being un-utilizable in the NMSGUT [23] also give a distinct hierarchy of right-handed neutrino masses. In our fits we find that right-handed neutrinos are much lighter than the GUT scale and strongly hierarchical while neither statement applies to the fits of [21,22]. Moreover [23] the translation between generic and NMSGUT parameters implies the NMSGUT parameters are subject to insolubly non-linear constraints rendering the usefulness of the generic fits problematic. We showed that in order to obtain fits that were compatible with the NMSGUT it would be necessary to search for fits using the detailed NMSGUT specific formulae developed by us in [25] since the the generic parameters 
used in [20, 21] were in fact subject to highly non-trivial constraints (due to their origin in the NMSGUT) that were in practice unverifiable since they could not be inverted.

Thus the GUT based on the $\mathbf{2 1 0} \oplus \mathbf{1 0} \oplus \mathbf{1 2 0} \oplus \mathbf{1 2 6} \oplus \overline{\mathbf{1 2 6}}$ Higgs system emerges as a New Minimal Supersymmetric GUT (NMSGUT) capable of fitting all the known fermion mass and mixing data. The New MSGUT calls for and deserves the same detailed analysis of its superheavy Renormalization Group(RG) flow threshold effects, fermion mass fit compatibility and exotic effect effective superpotential that we earlier provided [3, 6, 10, 15, 16] for the theory without the $\mathbf{1 2 0 .}$ We previously called that the MSGUT [12] but it's claim on that name is now tenuous and faded. In this paper we present the required spontaneous symmetry breaking, spectra, Higgs doublet fine-tuning and "Higgs-fraction" determination leading to matter fermion Yukawa expressions in terms of GUT parameters (as well as Weinberg operator coefficients leading to seesaw neutrino masses) and superheavy threshold effect formulae in the gauge RG flow from $M_{Z}$ up to $M_{X}$. With these formulae in hand we can face the burning question as to whether the renormalizable supersymmetric SO(10) GUT based on the Higgs set $\mathbf{2 1 0} \oplus \mathbf{1 0} \oplus \mathbf{1 2 0} \oplus \mathbf{1 2 6} \oplus \overline{\mathbf{1 2 6}}$ i.e. the socalled Next or New Minimal Supersymmetric GUT(NMSGUT) [25] is capable of encompassing the available fermion mass-mixing data.

As mentioned the fit to charged fermion data using only $\mathbf{1 0}$ and $\mathbf{1 2 0}$ representations fails badly to account for the down and strange quark Yukawa couplings at $M_{X}$. These Yukawas emerge much too small if the 3rd generation is fitted correctly. In retrospect this failure was to be expected since the $\overline{\mathbf{1 2 6}}$ contains the Higgs irreps required to implement the Georgi-Jarlskog [26] mechanism that can account for $y_{\mu} / y_{s}\left(M_{X}\right) \sim 3$ in terms of the vev of the $(15,1,1)$ sub-rep of the $\overline{\mathbf{1 2 6}}$ and third generation Yukawas dominated by the $\mathbf{1 0}$-plet. This is not possible when the $\overline{\mathbf{1 2 6}}$ couplings are highly suppressed.

Till recently these discussions -in the 'MSGUT community' - proceeded under the somewhat sanguine assumption that the appropriate uncertainties for the fitting exercise could be estimated by ignoring all threshold corrections right up to the GUT scale and hence were essentially the extrapolated errors at the low scale : thus for example the lepton masses were sought to be fitted to one part in a million or better [8. In fact however it has been known [27,28] from the early days of (large $\tan \beta) b-\tau-t$ unification that the $T_{3 L}=-1 / 2$ charged fermions suffer large threshold corrections. The relevance of these corrections for estimating the uncertainties in the GUT scale fermion masses has recently been re-emphasized [29, 30]. The NMSGUT like other $S O(10)$ GUTs prefers large $\tan \beta$ to naturally explain third generation charged fermion Yukawas $\left(y_{t}, y_{b}, y_{\tau}\right)$ as nearly equal due to their domination by the 33 component of the 10-plet Yukawa coupling. In this paper we shall see that the NMSGUT also requires large $\tan \beta \sim 50$ to drive threshold corrections that lower the values of the down and strange quark Yukawa couplings in the MSSM by a factor of about 5 while preserving, or rather slightly raising, the bottom quark Yukawa coupling in the MSSM. This allows us to match the rather low values (at $M_{X}^{0}=10^{16.33}$ $\mathrm{GeV}$ ) found by our "downhill simplex" search and fitting program. We run the couplings between $M_{Z}$ and $M_{X}^{0}$ using the two-loop Renormalization Group(RG) equations for the MSSM [31,39]; we also include running of the $d=5$ Weinberg operator [32, 33] but not any right-handed neutrino thresholds. This fitting determines characteristic patterns(but not the overall scale) of Susy breaking parameters and thus a distinctive phenomenology for the NMSGUT which is potentially falsifiable or verifiable at the LHC.

A significant improvement relative to the initial version of this paper [37 is that we carried out the fitting in terms of soft Susy breaking parameters at $M_{X}$ that manifestly respect the $\mathrm{SO}(10)$ GUT structure. We generated the soft Susy breaking terms from the values of $\left\{m_{0}, m_{1 / 2}, A_{0}, B, M_{H, \bar{H}}^{2}\right\}\left(M_{X}^{0}\right)$ together with $\mu\left(M_{X}^{0}\right)$ by imposing electroweak symmetry breaking (at one loop) at $M_{Z}$. These Susy breaking parameters respect the GUT symmetry. The light Higgs masses derive from the soft masses of several NMSGUT Higgs multiplets and may thus 
justifiedly taken to be different and independent of the sfermion mass parameter. The two scale fitting is achieved by the usual iterative process that loops from low to high scales and back [39]. The constraints determined by the peculiar route taken by NMSGUT fits may become acute if LHC discovers at least one of the Susy particles and anchors the floating scale of the Susy breaking soft mass patterns required in our model. Current [34] LHC data indicates that if a light Higgs exists it must be in the Susy preferred range of $115-145 \mathrm{GeV}$. In addition colored sparticles should be heavier than at least $500 \mathrm{GeV}$ and singly charged exotics than $200 \mathrm{GeV}$. Our analysis predicts a scenario with light $\operatorname{Bino} \operatorname{LSP}\left(\chi^{0} \sim \tilde{B}\right)$, light $\operatorname{Higgs}\left(h^{0}\right)$ and $\tilde{W}^{ \pm}$, heavy third generation sfermions, very heavy exotic Higgs(inos) $\left(A, H^{0}, H^{ \pm}, \chi_{3,4}^{0}\right)$ and with 1-2 generation sfermions spanning the range between the gaugino masses and the third sgeneration. In our model this novel pattern is made possible by values of $\mu, A_{0}, \sqrt{B} \sim 100 \mathrm{TeV}$. Since accurate unification is the main motivation for supersymmetry itself, the need at this juncture is for GUT driven insight into the possible mass patterns of Susy partners that might be unveiled by LHC. We offer the preferred soft parameter patterns of the NMSGUT as our 'bet' in the LHC-Susy 'sweepstakes'.

Even after it fits the fermion data the NMSGUT must still face the challenge posed by the non-observation of proton decay. Minimal Susy GUTs generically imply proton decay rates via $d=5$ operators which are higher than the current experimental upper bounds [35] (but see also [36]). Soft susy breaking parameters and in particular the sfermion masses and mixing matrices have a crucial influence on the rates of the $d=5$ mediated proton decay. In this paper we derive also the $\Delta B \neq 0$ effective superpotential by integrating out heavy fields and obtain the (operator dimension 4) B violation superpotential. We use these to calculate the nucleon decay rates implied by the fermion fit parameters found. The rapidity of the baryon decay rates presents a difficulty whose resolution requires further development of the NMSGUT to account for threshold effects also on the GUT scale effective Yukawa couplings which is accomplished in a subsequent paper [38].

In Section 2 We discuss the modifications of the superpotential introduced by the $\mathbf{1 2 0}$-plet. For completeness some details regarding the parameters of the MSGUT superpotential are summarized in Appendix A. In Section $\mathbf{3}$ we discuss the modified mass spectra of the NMSGUT and give the detailed analytic expressions in Appendix B. In Section 4 we give the renormalization group formulae used to check the effect of superheavy thresholds on the unification parameters. In section 5 we give formulae relating the effective MSSM parameters in terms of $\mathrm{SO}(10)$ parameters. In sub-section $\mathbf{5 . 1}$ we give the formulae for the fermion Yukawas of the effective MSSM in terms of the GUT couplings and the so-called "Higgs fractions". In sub-section $\mathbf{5 . 2}$ we integrate out the heavy triplets that mediate baryon decay via $d=5$ operators and give the resultant effective superpotential in terms of the matter superfields of the effective MSSM. In Section 6 we describe our numerical procedure but give details regarding Susy threshold corrections and electroweak breaking in Appendix C. In Section 7 we discuss(Section 7.1) the features of two example fits (the numerical parameter values of which are given in tabular form in Appendix D) and dissect(Section 7.2) their structure. In Section $\mathbf{7 . 3}$ we use the explicit sample sets of NMSGUT parameter values and Susy soft breaking data to estimate typical predictions of a fully specified NMSGUT for nucleon decay rates using the formalism developed in [40,41] (and by earlier workers cited therein). We conclude, in Section 8, with a summary, a discussion of issues and the way forward. [38].

\section{The New Minimal Susy GUT}

The original MSGUT [10 12, 14, was the renormalizable supersymmetric $S O(10)$ GUT whose Higgs chiral supermultiplets consist of AM(Adjoint Multiplet) type totally antisymmetric tensors : $\mathbf{2 1 0}\left(\Phi_{i j k l}\right), \overline{\mathbf{1 2 6}}\left(\overline{\boldsymbol{\Sigma}}_{i j k l m}\right), \mathbf{1 2 6}\left(\boldsymbol{\Sigma}_{i j k l m}\right)(i, j=1 \ldots 10)$ which break the GUT symmetry to the 
MSSM, together with Fermion mass (FM) Higgs 10-plet $\left(\mathbf{H}_{i}\right)$. The $\overline{\mathbf{1 2 6}}$ plays a dual or AMFM role since it also enables the generation of realistic charged fermion and neutrino masses and mixings (via the Type I and/or Type II seesaw mechanisms); three $\mathbf{1 6}$-plets $\mathbf{\Psi}_{\mathbf{A}}(A=1,2,3)$ contain the matter including the three conjugate neutrinos $\left(\bar{\nu}_{L}^{A}\right)$. Some further details are provided in Appendix $\mathbf{A}$ and a full treatment is available in [3, 4, 6, 12,

The introduction of the three anti-symmetric index 120-plet Higgs representation $\Theta_{i j k}$ leads to new couplings in the superpotential. The additional terms are

$$
\begin{aligned}
W_{N M S G U T} & =\frac{m_{\Theta}}{2(3 !)} \Theta_{i j k} \Theta_{i j k}+\frac{k}{3 !} \Theta_{i j k} H_{m} \Phi_{m i j k}+\frac{\rho}{4 !} \Theta_{i j k} \Theta_{m n k} \Phi_{i j m n} \\
& +\frac{1}{2(3 !)} \Theta_{i j k} \Phi_{k l m n}\left(\zeta \Sigma_{l m n i j}+\bar{\zeta} \bar{\Sigma}_{l m n i j}\right)+\frac{1}{3 !} g_{A B} \Psi_{A}^{T} C_{2}^{(5)} \gamma_{i} \gamma_{j} \gamma_{k} \Psi_{B} \Theta_{i j k}
\end{aligned}
$$

The Yukawa coupling $g_{A B}$ is a complex antisymmetric $3 \times 3$ matrix. We will frequently need to decompose $\mathrm{SO}(10)$ fields and invariants according to the Pati-Salam and SM subgroups of $\mathrm{SO}(10)$. This involves translating the $\mathrm{SO}(10)$ vector and spinor labels to those of these subgroups. A complete method and handbook for this translation is available in [3. Some relevant details about our index conventions are as follows : The real indices of the vector representation of $\mathrm{SO}(10)$ are denoted by $i, j=1 . .10$. The indices of the doublet of $\mathrm{SU}(2)_{L}\left(\mathrm{SU}(2)_{\mathrm{R}}\right)$ are denoted $\alpha, \beta=1,2(\dot{\alpha}, \dot{\beta}=\dot{1}, \dot{2})$. Finally the index of the fundamental 4-plet of $\mathrm{SU}(4)$ is denoted by a (lower) $\mu, \nu=1,2,3,4$ and its upper-left block $\mathrm{SU}(3)$ subgroup indices are $\bar{\mu}, \bar{\nu}=1,2,3$. The corresponding indices on the $\overline{4}$ are carried as superscripts. These doublets and quartets correspond to the chiral spinor representations of the $S O(4)$ and $S O(6)$ subgroups of $S O(10)$. Details of the spinor decomposition techniques may be found in [3]. The $S U(4) \times S U(2)_{L} \times S U(2)_{R} \quad((n, m, l)$ denotes a Pati-Salam representation of $\mathrm{SU}(4)$ dimension n,$S U(2)_{L}$ dimension $\mathrm{m}$ and $S U(2)_{R}$ dimension $l$ ) decomposition of the $\mathbf{1 2 0}$-plet is as follows :

$$
\begin{aligned}
\Theta_{i j k}(120) & =\Theta_{\mu \nu}^{(s)}(10,1,1)+\Theta_{(s)}^{\mu \nu}(\overline{10}, 1,1)+\Theta_{\nu \alpha \dot{\alpha}}{ }^{\mu}(15,2,2) \\
& +\Theta_{\mu \nu \dot{\alpha} \dot{\beta}}^{(a)}(6,1,3)+\Theta_{\mu \nu}^{(a)}(6,3,1)+\Theta_{\alpha \dot{\alpha}}(1,2,2)
\end{aligned}
$$

The sub/superscripts "(s), (a)" denote symmetry and antisymmetry in SU(4) indices $\mu, \nu$. Note that the 120-plet contains no SM singlet so that the MSGUT high scale spontaneous symmetry breaking analysis remains the same. The arbitrary phase of the $\mathbf{1 2 0}$ reduces the effective number of the extra couplings $\left(m_{\Theta}, \rho, \zeta, \bar{\zeta}, k\left(5_{c}-1_{r}=9_{r}\right)\right.$ and $\left.g_{A B}\left(3_{c}=6_{r}\right)\right)$ so they amount to 15 additional parameters. Thus the relative advantage [12,42] with respect to $S U(5)$ theories using additional fields or higher dimensional operators to correct the fermion mass relations of the simplest $S U(5)$ model seems weakened but is not lost.

In fact the old MSGUT fails to fit the fermion mass data due to difficulties with the overall neutrino mass scale [15,16]. An alternative scenario within the NMSGUT to remove the problems of the old MSGUT was proposed and elaborated in [16, 43, 44]. In this scenario the Yukawa couplings $\left(f_{A B}\right)$ of the $\overline{\mathbf{1 2 6}}$ are much smaller than those of the 10,120. This boosts the value of the Type I seesaw masses (which were in any case dominant over the Type II seesaw masses but still too small) so that they are generically viable.

The NMSGUT even with only real Yukawas(except the fine tuned complex parameter $M_{H}$ ) i.e. with a total of $23(=12$ fermion Yukawas +11 AM Yukawas) real parameters (further reduced to 22 by the fine tuning condition ) may be first tried to fit the fermion data ( 12 masses + CKM phase +3 CKM angles +3 PMNS angles +3 PMNS phases $=22$ parameters, of which the last three and one neutrino mass are unknown leaving 18 targets for the fitting). Such a theory will have less parameters than even the (unsuccessful) old MSGUT. However restriction to real values 
is somewhat arbitrary since it cannot be justified by a CP symmetry in view of the complexity of the fine tuned value of $M_{H}$. A preliminary search with real parameters is useful for locating promising approximate solutions to use as starting points in the full parameter space.Thus we shall allow all parameters to be complex. In that case number of free NMSGUT superpotential parameters mounts to 37 .

The FM Higgs 120 does not contain any SM singlets and hence the analysis of the GUT scale symmetry breaking in MSGUT carries over unchanged to the NMSGUT. In particular there is still only one complex parameter $(x)$ whose variation directly affects the vevs and thus the masses in the theory. The additional kinetic terms are given by covariantizing in the standard way the global SO(10) invariant D-terms $\left[\frac{1}{3 !} \boldsymbol{\Theta}_{i j k}^{*} \boldsymbol{\Theta}_{i j k}\right]_{D}$.

\section{AM Chiral masses via PS}

As in the case of the MSGUT [3, 6] we open up the maze of NMSGUT interactions by decomposing $S O(10)$ invariants in the superpotential first into Pati-Salam invariants and then, after substituting the GUT scale vevs, we obtain the superpotential in the MSSM vacuum in terms of MSSM invariants. The results for the old MSGUT case were already given in [3, 6] thus we list only the effect of the additional terms in the superpotential.

An example of the PS form of $W_{N M S G U T}$ is (we have inserted line numbers for easy reference):

$$
\begin{aligned}
\frac{k}{3 !} H_{i} \Theta_{j k l} \Phi_{i j k l}= & k\left[\frac{1}{\sqrt{2}} i\left(\tilde{H}_{(a)}^{\mu \nu} \Theta_{\mu \lambda}^{(s)} \Phi_{\nu}{ }^{\lambda}+H_{\mu \nu}^{(a)} \Theta_{(s)}^{\mu \lambda} \Phi_{\lambda}{ }^{\nu}\right)\right. \\
+ & \frac{1}{\sqrt{2}}\left(\tilde{H}_{(a)}^{\mu \nu} \Theta_{\nu}^{\lambda \alpha \dot{\alpha}} \Phi_{\mu \lambda \alpha \dot{\alpha}}^{(s)}-H_{\mu \nu}^{(a)} \Theta_{\lambda}^{\nu \alpha \dot{\alpha}} \Phi_{\alpha \dot{\alpha}}^{\mu \lambda(s)}\right) \\
& +H_{\mu \nu}^{(a)}\left(\vec{\Theta}_{(a)(R)}^{\nu \lambda} \cdot \vec{\Phi}_{\lambda(R)}^{\mu}+\vec{\Theta}_{(a)(L)}^{\nu \lambda} \cdot \vec{\Phi}_{\lambda(L)}^{\mu}\right) \\
& -\frac{1}{2} \tilde{H}_{(a)}^{\mu \nu} \Theta^{\alpha \dot{\alpha}} \Phi_{\mu \nu \alpha \dot{\alpha}}^{(a)} \\
& +\frac{1}{2} H^{\alpha \dot{\alpha}}\left(\Theta_{\mu \nu}^{(s)} \Phi_{\alpha \dot{\alpha}}^{\mu \nu(s)}+\Theta_{(s)}^{\mu \nu} \Phi_{\mu \nu \alpha \dot{\alpha}}^{(s)}\right) \\
& -\frac{1}{\sqrt{2}} H^{\alpha \dot{\alpha}}\left(\Theta_{\nu \alpha}^{\mu \dot{\beta}} \Phi_{\mu \dot{\alpha} \dot{\beta}}^{\nu}+\Theta_{\nu \dot{\alpha}}^{\mu \beta} \Phi_{\mu \alpha \beta}^{\nu}\right) \\
& -\frac{1}{2 \sqrt{2}} H^{\alpha \dot{\alpha}}\left(\tilde{\Theta}_{(a) \dot{\alpha} \dot{\beta}}^{\mu \nu} \Phi_{\mu \nu \alpha}^{(a) \dot{\beta}}-\tilde{\Theta}_{(a) \alpha \beta}^{\mu \nu} \Phi_{\mu \nu \dot{\alpha}}^{(a) \beta}\right) \\
& \left.+H^{\alpha \dot{\alpha}} \Theta_{\alpha \dot{\alpha}} \Phi\right]
\end{aligned}
$$

These terms are now easy to decompose into MSSM invariants using the decompositions $S U(4) \rightarrow$ $S U(3) \times U(1)_{B-L}$ and the weak hypercharge identification $Y=2 T_{3 R}+B-L$ in the left-right symmetric gauge group. The decomposition of the rest of the superpotential terms due to the 120-plet is given in the second part of Appendix A.

The purely chiral superheavy supermultiplet masses can be determined from these expressions simply by substituting in the AM Higgs vevs and breaking up the contributions according to MSSM labels.

It is again easiest to keep track of Chiral fermion masses since all others follow using supersymmtery and the organization provided by the gauge super-Higgs effect.

There are three types of mass terms involving fermions from chiral supermultiplets in such models :

- Unmixed Chiral 
- Mixed pure chiral

- Mixed chiral-gauge. We briefly discuss the notable features of the mass spectrum calculation and give the actual mass formulae in the Appendix $\mathbf{B}$.

\subsection{Unmixed Chiral}

There are 26 different SM multiplet types in the MSGUT and NMSGUT. Thus the alphabetical naming convention specified in Appendix $\mathbf{B}$ is very natural. A pair of chiral fermions transforming as $S U(3) \times S U(2)_{L} \times U(1)_{Y}$ conjugates pairs up to form a massive Dirac fermion. In the MSGUT case there are 17 MSSM types of unmixed chiral multiplets which form Dirac supermultiplets pairwise and two Majorana singletons. In the NMSGUT 6 of these types (C,D,E,K,L,P) become further mixed with others of the same type leaving 11 Dirac supermultiplets (A,B,I,M,N,O,U,V,W,Y,Z) and 2 Majorana supermultiplets $(\mathrm{S}, \mathrm{Q})$ unmixed. If the representation is real rather than complex one obtains an extra factor of 2 in the masses. The representations, field components and masses of all fields are given in in Appendix $\mathbf{B}$.

\subsection{Mixed Pure Chiral}

For such multiplets there is no mixing with the massive coset gauginos but there is a mixing among several multiplets with the same SM quantum numbers. There were only three such multiplet types in the MSGUT (i.e. $R[8,1,0], h[1,2, \pm 1], t\left[3,1, \pm \frac{2}{3}\right]$ ) but in the NMSGUT, there are an additional 5 mixed pure chiral types namely the $C[8,2, \pm 1], D\left[3,2, \pm \frac{7}{3}\right], K\left[3,1, \pm \frac{8}{3}\right]$,

$L\left[6,1, \pm \frac{2}{3}\right], P\left[3,3, \pm \frac{2}{3}\right]$. As for the multiplet types which had mixed pure chiral mass terms in the MSGUT, the type $R[8,1,0]$ acquires no new partners and has an unchanged mass matrix since the $\mathbf{1 2 0}$ has no such submultiplets. However the other two mixed pure chiral multiplet types of the MSGUT do acquire new contributions :

- $[1,2,-1]\left(\bar{h}_{1}, \bar{h}_{2}, \bar{h}_{3}, \bar{h}_{4}, \bar{h}_{5}, \bar{h}_{6}\right) \bigoplus[1,2,1]\left(h_{1}, h_{2}, h_{3}, h_{4}, h_{5}, h_{6}\right) \equiv\left(H_{\dot{2}}^{\alpha}, \bar{\Sigma}_{\dot{2}}^{(15) \alpha}\right.$, $\left.\Sigma_{\dot{2}}^{(15) \alpha}, \frac{\Phi_{44}^{\dot{2} \alpha}}{\sqrt{2}}, \Theta_{\dot{2}}^{\alpha}, \Theta_{\dot{2}}^{(15) \alpha}\right) \bigoplus\left(H_{\alpha \dot{1}}, \bar{\Sigma}_{\alpha \dot{1}}^{(15)}, \Sigma_{\alpha \dot{1}}^{(15)}, \frac{\Phi_{\alpha}^{44 \dot{1}}}{\sqrt{2}}, \Theta_{\alpha \dot{1}}, \Theta_{\alpha \dot{1}}^{(15)}\right)$

Here one gets an additional 2 rows and 2 columns relative to the MSGUT since the 120-plet contains two pairs of doublets with MSSM type Higgs doublet quantum numbers so that the mass matrix $\mathcal{H}$ is $6 \times 6$. To keep one pair of light doublets in the low energy effective theory, it is necessary to fine tune one of the parameters of the superpotential (e.g. $M_{H}$ ) so that $\operatorname{Det} \mathcal{H}=0$. By extracting the null eigenvectors of $\mathcal{H}^{\dagger} \mathcal{H}$ and $\mathcal{H} \mathcal{H}^{\dagger}$ one can compute the composition of the light doublet pair in terms of the doublet fields in the full SO(10) GUT, and, in particular, we can find the proportions of the doublets coming from the 10, $\overline{\mathbf{1 2 6}}, \mathbf{1 2 0}$ multiplets which couple to the matter sector [25].

- $\left[\overline{3}, 1, \frac{2}{3}\right]\left(\bar{t}_{1}, \bar{t}_{2}, \bar{t}_{3}, \bar{t}_{4}, \bar{t}_{5}, \bar{t}_{6}, \bar{t}_{7}\right) \bigoplus\left[3,1,-\frac{2}{3}\right]\left(t_{1}, t_{2}, t_{3}, t_{4}, t_{5}, t_{6}, t_{7}\right) \equiv\left(H^{\bar{\mu} 4}, \bar{\Sigma}_{(a)}^{\bar{\mu} 4}, \Sigma_{(a)}^{\bar{\mu} 4}, \Sigma_{(R 0)}^{\bar{\mu} 4}\right.$, $\left.\Phi_{4(R+)}^{\bar{\mu}}, \Theta^{\bar{\mu} 4(s)}, \Theta_{(R 0)}^{\bar{\mu} 4}\right) \bigoplus\left(H_{\bar{\mu} 4}, \bar{\Sigma}_{\bar{\mu} 4(a)}, \Sigma_{\bar{\mu} 4(a)}, \bar{\Sigma}_{\bar{\mu} 4(R 0)}, \Phi_{\bar{\mu}(R-)}^{4}, \Theta_{\bar{\mu} 4(s)}, \Theta_{\bar{\mu} 4(R 0)}\right)$

With the contribution of the 120-plet one gets two additional rows and columns and the dimension of $\left[3,1, \pm \frac{2}{3}\right]$ mass matrix $\mathcal{T}$ becomes $7 \times 7$. These triplets and antitriplets participate in baryon violating processes since the exchange of $\left(t_{1}, t_{2}, t_{4}, t_{6}, t_{7}\right) \oplus\left(\bar{t}_{1}, \bar{t}_{2}, \bar{t}_{6}, \bar{t}_{7}\right)$ Higgsinos generates $d=5$ operators of type QQQL and $\bar{l} \bar{u} \bar{d}$. The strength of the operator is controlled by the inverse of the $\bar{t}-t$ mass matrix $\mathcal{T}$. 


\subsection{Mixed Chiral-Gauge}

Finally we come to the mixing matrices for the chiral modes that mix with the gauge particles as well as among themselves. There is no direct mixing between MSSM fields contained in 120plet with gauge particles. However mixing is present via other MSSM submultiplets present in NMSGUT Higgs fields which further mix with gauge fields. This occurs for all such multiplet types except $G[1,1,0]$ and $X\left[3,2, \pm \frac{4}{3}\right]$ which are unchanged, while for $E\left[3,2, \pm \frac{1}{3}\right], F[1,1, \pm 2], J\left[3,1, \pm \frac{4}{3}\right]$ mass matrices acquire additional rows and columns. Thus

- $\left[\overline{3}, 2,-\frac{1}{3}\right]\left(\bar{E}_{1}, \bar{E}_{2}, \bar{E}_{3}, \bar{E}_{4}, \bar{E}_{5}, \bar{E}_{6}\right) \oplus\left[3,2, \frac{1}{3}\right]\left(E_{1}, E_{2}, E_{3}, E_{4}, E_{5}, E_{6}\right)$ $\equiv\left(\Sigma_{4 \dot{1}}^{\bar{\mu} \alpha}, \bar{\Sigma}_{4 \dot{1}}^{\bar{\mu} \alpha}, \phi_{(s) \dot{2}}^{\bar{\mu} 4 \alpha}, \phi_{\dot{2}}^{(a) \bar{\mu} 4 \alpha}, \lambda_{\dot{2}}^{\bar{\mu} 4 \alpha}, \Theta_{4 \dot{1}}^{\bar{\sigma} \alpha}\right) \oplus\left(\bar{\Sigma}_{\bar{\mu} \alpha \dot{2}}^{4} \Sigma_{\bar{\mu} \alpha \dot{2}}^{4}, \phi_{\bar{\mu} 4 \alpha \dot{1}}^{(s)}, \phi_{\bar{\mu} 4 \alpha \dot{1}}^{(a)}, \lambda_{\bar{\mu} \alpha \dot{1}}, \Theta_{\bar{\sigma} \alpha}^{4 \dot{1}}\right)$

The $6 \times 6$ mass matrix $\mathcal{E}$ has the usual super-Higgs structure : complex conjugates of the 5 th row and column (omitting the diagonal entry) furnish left and right null eigenvectors of the chiral $5 \times 5$ submatrix $\mathbf{E}$ obtained by omitting the fifth row and column. $\mathcal{E}$ has non-zero determinant although the determinant of $\mathbf{E}$ vanishes.

- $[1,1,-2]\left(\bar{F}_{1}, \bar{F}_{2}, \bar{F}_{3}, \bar{F}_{4}\right) \bigoplus[1,1,2]\left(F_{1}, F_{2}, F_{3}, F_{4}\right) \equiv\left(\bar{\Sigma}_{44(R 0)} / \sqrt{2}, \Phi_{(R-)}^{(15)}, \lambda_{(R-)}\right.$, $\left.\Theta_{44} / \sqrt{2}\right) \bigoplus\left(\Sigma_{(R 0)} / \sqrt{2}, \Phi_{(R+)}^{(15)}, \lambda_{(R+)}, \Theta^{44} / \sqrt{2}\right)$

The same comments as for $E\left[3,2, \pm \frac{1}{3}\right]$ apply mutatis mutandis i.e. $4 \times 4$ mass matrix $\mathcal{F}$ with $3 \times 3$ submatrix $\mathbf{F}$ and so forth.

- $\left[\overline{3}, 1,-\frac{4}{3}\right]\left(\bar{J}_{1}, \bar{J}_{2}, \bar{J}_{3}, \bar{J}_{4}, \bar{J}_{5}\right) \oplus\left[3,1, \frac{4}{3}\right]\left(J_{1}, J_{2}, J_{3}, J_{4}, J_{5}\right)$ $\equiv\left(\Sigma_{(R-)}^{\bar{\mu} 4}, \phi_{4}^{\bar{\mu}}, \phi_{4}^{\bar{\mu}(R 0)}, \lambda_{4}^{\bar{\mu}}, \Theta_{(R-)}^{\bar{\mu} 4}\right) \oplus\left(\bar{\Sigma}_{\bar{\mu} 4(R+)}, \phi_{\bar{\mu}}^{4}, \phi_{\bar{\mu}(R 0)}^{4}, \lambda_{\bar{\mu}}^{4}, \Theta_{\bar{\mu} 4}^{(R+)}\right)$

Here we have $5 \times 5$ mass matrix $\mathcal{J}$ with $4 \times 4$ submatrix $\mathbf{J}$ and so forth.

This concludes our description of the superheavy mass spectrum of the NMSGUT.

\section{RG Analysis}

The analysis of the flow of gauge couplings is the most familiar element in papers on grand unification, with the iconic diagram of merging gauge couplings at the GUT scale as an easily recognized referent. Even the failure to unify is often quantified in terms of a failure of the three running couplings to intersect at a point. However in the conceptually clearer approach of Weinberg [45], explicated at length by Hall [46], based upon matching the low and high energy effective gauge field theories in an overlap region, the flow of the three gauge couplings of the SM intersects the flow of the gauge coupling of the unifying group at three distinct scales. Nevertheless the three equations relating the MSSM gauge couplings at $M_{Z}$ to the GUT coupling at any convenient superheavy scale may be solved for the unification predictions. Perturbative consistency demands that the three scales are closely spaced so that the calculations are consistent. To counter the fixed idea that the aim of unification is a three fold intersection of gauge couplings we explain the formalism and our procedure at some length. With the superheavy spectrum formulae in hand the threshold corrections to the matching relations between the couplings in the low and high energy effective theories can be evaluated. The relation between the MSSM couplings at the low scale where they are specified from measurement (which we always take to be the $\mathrm{Z}$ boson mass $M_{Z}=91.1876 \mathrm{GeV}$ to allow easy use of the standard calculation of 1-loop effects in the MSSM [60]) and the GUT coupling at the scale $M_{X}$ is given by [45, 46] :

$$
\frac{1}{\hat{\alpha}_{i}\left(M_{Z}\right)}=\frac{1}{\alpha_{G}\left(M_{X}\right)}+8 \pi b_{i} \ln \frac{M_{X}}{M_{Z}}+4 \pi \sum_{j} \frac{b_{i j}}{b_{j}} \ln X_{j}-4 \pi \lambda_{i}\left(M_{X}\right)+\ldots
$$


Here $\hat{\alpha}_{i}=g_{i}^{2} / 4 \pi, i=1,2,3$, where $g_{i}$ are the MSSM gauge couplings in (SU(5)) GUT normalization, should not be confused with the Higgs fractions $\alpha_{i}, i=1 \ldots 6$. The second, third, and fourth terms correspond to the one-loop gauge running, two loop gauge running and superheavy threshold corrections respectively. The ellipsis refers to the (small) contributions from matter Yukawa couplings at two loops. Here $X_{j}=1+8 \pi b_{j} \alpha_{G}\left(M_{X}\right) \ln \frac{M_{X}}{M_{Z}}$ and $\lambda_{i}\left(M_{X}\right)$ may be evaluated at any superheavy scale (concretely : any scale whose logarithm differs from that of the 1-loop $\operatorname{crossing} \operatorname{point}\left(M_{X}^{0}\right)$ of the $S U(2)$ and $U(1)$ couplings by terms of order $\alpha[46]$ ). We shall use $M_{X}^{0}$ as the leading approximation to the superheavy matching scale between MSSM and GUT in all our calculations, while the unification scale itself is taken as the mass $M_{X}$ of the $X[3,2, \pm 5 / 3]$ type gauge supermultiplet. Similarly for ease of interpretation we shall use the mass of the Z boson $M_{Z}=91.1876 \mathrm{GeV}$ as the low scale renormalization and matching point between the $\mathrm{SM}$ and MSSM. The coefficients $b_{i}\left\{b_{1}, b_{2}, b_{3}\right\}=\left(1 / 16 \pi^{2}\right)\left\{\frac{33}{5}, 1,-3\right\}$ are the one-loop gauge beta function coefficients in the MSSM and similarly $b_{i j}$ are the two-loop coefficients [31]. The term containing $\lambda_{i}$ represents the leading contribution of the superheavy thresholds calculated within the Weinberg-Hall formalism [45,46]. In the $\overline{M S}$ scheme one has :

$$
\lambda_{i}(\mu)=-\frac{2}{21}\left(b_{i V}+b_{i G B}\right)+2\left(b_{i V}+b_{i G B}\right) \ln \frac{M_{V}}{\mu}+2 b_{i S} \ln \frac{M_{V}}{\mu}+2 b_{i F} \ln \frac{M_{F}}{\mu}
$$

where V,GB,S,F refer to vectors, Goldstone bosons, scalars and fermions respectively and $b_{i}$ are one-loop beta function coefficients, and a sum over heavy mass eigenstates is implicit. The coefficient of the $\mu$ independent term arises [46] in an $\overline{M S}$ scheme where metric traces are carried out in $D-4$ but the gamma matrix traces for fermions are in 4 dimensions. However for supersymmetric theories the $\overline{D R}$ scheme [47] should be used so that the equality of bosonic and fermionic degrees of freedom is preserved. In this scheme only the momentum integrations, but not the metric or gamma matrix traces, are continued away from 4 dimensions. Hence the $\mu$ independent term is absent from the threshold corrections in the $\overline{D R}$ scheme. Thus for consistency we should convert the input parameters $\alpha_{1,2}\left(M_{Z}\right)$ calculated from the precisely known [48] electroweak data in the $\overline{M S}$ scheme $\left(1 / \alpha\left(M_{Z}\right)=127.916 \pm 0.015, \sin ^{2} \theta_{W}=0.23116 \pm 0.00013\right)$ into the corresponding $\overline{D R}$ parameters.

Next one uses the three equations to determine $\hat{\alpha}_{3}\left(M_{Z}\right), M_{X}, \alpha_{G}\left(M_{X}\right)$ as a perturbation expansion around the one-loop values. In view of the above remarks the unified coupling $\alpha_{G}$ and the prediction for $\hat{\alpha}_{3}\left(M_{Z}\right)$ will also emerge in the $\overline{D R}$ scheme. The required formulae were conveniently summarized in [49] and analysis of the difficulty concerning a too high value of $\hat{\alpha}_{3}\left(M_{Z}\right)$ can be found in [50].

Inserting the $\overline{D R}$ converted values we find the one-loop predictions

$$
M_{X}^{0}=10^{16.33} \mathrm{GeV} \quad\left(\alpha_{G}^{0}\right)^{-1}=24.2496 \quad \hat{\alpha}_{3}^{0}\left(M_{Z}\right)=0.1183
$$

Including the two-loop corrections and iterating once gives the two-loop values

$$
M_{X}=10^{16.553} \mathrm{GeV} \quad \alpha_{G}^{-1}=22.9803 \quad \hat{\alpha}_{3}\left(M_{Z}\right)=0.1307
$$

This should be compared with the $\overline{D R}$ experimental value $\left(\hat{\alpha}_{3}\left(M_{Z}\right)\right)^{\text {expt }}=0.1194$. Two-loop corrections $H_{s}\left(y_{t}, y_{b}, y_{\tau}, \ldots, \tan \beta\right)$ due to third generation Yukawas are usually negative but typically [50] $H_{s}>-.003$ even for the large values of $\tan \beta \sim 50$ relevant for $\mathrm{SO}(10)$ Susy GUTs. Thus it is clear that there is, prima facie, a discrepancy of 6 to $8 \%$ between the larger value of the prediction and the current best estimate for $\hat{\alpha}_{3}\left(M_{Z}\right)^{\overline{D R}}=0.119$. However the effects of threshold corrections due to the supersymmetric thresholds between $M_{Z}$ and (up to, say,) $100 \mathrm{TeV}$ as well as the thresholds associated with the very large number $(\sim 500)$ superheavy fields present in the model (with masses ranging in the band $10^{15 \pm 3} \mathrm{GeV}$ ) could well explain this discrepancy. It is the 
effect of superheavy thresholds that is the focus of the gauge RG investigations of the NMSGUT in this paper. With the above motivation we shall search for GUT parameter sets for which the corrections to the unification parameters due to superheavy thresholds stay within the following prima facie reasonable but open-minded limits :

$$
\begin{aligned}
-20.0 \leq \Delta_{G} & \equiv \Delta\left(\alpha_{G}^{-1}\left(M_{X}\right)\right) \leq 25 \\
3.0 \geq \Delta_{X} & \equiv \Delta\left(\log _{10} M_{X}\right) \geq-0.3 \\
-0.017<\Delta_{3} & \equiv \hat{\alpha}_{3}\left(M_{Z}\right)<-0.004
\end{aligned}
$$

These limits are imposed to respect perturbativity, $M_{X}<M_{\text {Planck }}$ and yield a negative correction to $\hat{\alpha}_{3}\left(M_{Z}\right)$ in the relevant range. Here $\Delta_{G, X}$ include the two-loop corrections as well as the threshold corrections but $\Delta_{3}$ is only due to superheavy thresholds. Eventually- when likely supersymmetric thresholds are also established - we can combine all contributions in order to fit $\hat{\alpha}_{3}\left(M_{Z}\right)$ to within its error bars (currently given, somewhat optimistically at around $1.5 \%$ but with a rather larger variance across measurement methods!).

The threshold correction [6, 15, formulae are

$$
\begin{aligned}
\Delta^{(t h)}\left(\ln M_{X}\right) & =\frac{\lambda_{1}\left(M_{X}\right)-\lambda_{2}\left(M_{X}\right)}{2\left(b_{1}-b_{2}\right)} \\
\Delta_{X} & \equiv \Delta^{(t h)}\left(\log _{10} \frac{M_{X}}{1 G e V}\right)+\Delta^{(2-l o o p)}\left(\log _{10} \frac{M_{X}}{1 G e V}\right) \\
& =0.222+\frac{5\left(\bar{b}_{1}^{\prime}-\bar{b}_{2}^{\prime}\right)}{28} \log _{10} \frac{M^{\prime}}{M_{X}} \\
\Delta_{3} & \equiv \Delta^{(t h)}\left(\hat{\alpha}_{3}\left(M_{Z}\right)\right) \\
& =\frac{100 \pi\left(b_{1}-b_{2}\right) \alpha\left(M_{Z}\right)^{2}}{\left[\left(5 b_{1}+3 b_{2}-8 b_{3}\right) \sin ^{2} \theta_{w}\left(M_{Z}\right)-3\left(b_{2}-b_{3}\right)\right]^{2}} \sum_{i j k} \epsilon_{i j k}\left(b_{i}-b_{j}\right) \lambda_{k}\left(M_{X}\right) \\
& =.000311667 \sum_{M^{\prime}}\left(5 \bar{b}_{1}^{\prime}-12 \bar{b}_{2}^{\prime}+7 \bar{b}_{3}^{\prime}\right) \ln \frac{M^{\prime}}{M_{X}} \\
\Delta_{G} & \equiv \Delta^{(t h)}\left(\alpha_{G}^{-1}\left(M_{X}\right)\right)+\Delta^{(2-l o o p)}\left(\alpha_{G}^{-1}\left(M_{X}\right)\right)=\frac{4 \pi\left(b_{1} \lambda_{2}\left(M_{X}\right)-b_{2} \lambda_{1}\left(M_{X}\right)\right)}{b_{1}-b_{2}} \\
& =-1.27+\frac{1}{56 \pi} \sum_{M^{\prime}}\left(33 \bar{b}_{2}^{\prime}-5 \bar{b}_{1}^{\prime}\right) \ln \frac{M^{\prime}}{M_{X}}
\end{aligned}
$$

Where $\bar{b}_{i}^{\prime}=16 \pi^{2} b_{i}^{\prime}$ are 1-loop $\beta$ function coefficients $\left(\beta_{i}=b_{i} g_{i}^{3}\right)$ for multiplets with mass $M^{\prime}$ and $\lambda_{i}$ are the leading contributions of the superheavy thresholds [6, 46].

We identify the scale $M_{X}$ with the physical mass of the gauge supermultiplet transforming as $\left[3,2, \pm \frac{5}{3}\right]$ responsible for $d=6$ baryon violation. A crucial point [15] is that the threshold corrections depend only on ratios of masses and are independent of the overall scale parameter which we choose to be the mass parameter of the 210-plet $m$. Since on the one hand $M_{X}=$ $M_{X}^{0} 10^{\Delta_{X}}$ and on the other $M_{X}=m_{\lambda_{X}}=|m / \lambda| g \sqrt{4|\tilde{a}+\tilde{w}|^{2}+2|\tilde{p}+\tilde{\omega}|^{2}}$ it follows that the parameter $m$ is determined by the RG analysis to be :

$$
\begin{aligned}
\Delta_{X} & =\Delta\left(\log _{10} \frac{M_{X}}{1 G e V}\right) \\
|m| & =M_{X}^{0} 10^{+\Delta_{X}} \frac{|\lambda|}{g \sqrt{4|\tilde{a}+\tilde{w}|^{2}+2|\tilde{p}+\tilde{\omega}|^{2}}} G e V
\end{aligned}
$$

In the (N)MSGUT $m$ sets the scale of all the superheavy vevs so every superheavy mass must rise or fall in tandem with $M_{X}$ i.e with $\Delta_{X}$. The $\mathrm{SO}(10)$ gauge coupling in this formula may be improved by using its threshold corrected value. 
These corrections modify the one-loop values corresponding to the successful gauge unification of the MSSM. In spite of the large number of superheavy fields they can still give viable unification over extended regions of the GUT parameter space thus belying [6, 15, 16]) early expectations that the unification exercise is futile in $\mathrm{SO}(10)$ Susy GUTs [54]. The parameter $\xi=\lambda M / \eta m$ (see Appendix A of this paper for the parameter naming) is the only numerical parameter that enters into the cubic eqn. (A.8) that determines the parameter $x$ in terms of which all the superheavy vevs are given. It is thus the most crucial determinant of the mass spectrum. The rest of the coupling parameters divide into "diagonal" $(\lambda, \eta, \rho)$ and "non-diagonal" $(\gamma, \bar{\gamma}, \zeta, \bar{\zeta}, k)$ couplings with the latter exerting a weaker influence on the unification parameters [6, 25]. The dependence of the threshold corrections on the "diagonal" couplings is also comparatively mild except when coherent e.g when many masses are lowered together leading to $\alpha_{G}$ explosion, $\log M_{X}$ collapse or large changes in $\hat{\alpha}_{3}\left(M_{Z}\right)$. The development of the NMSGUT presented here was motivated by the need to reconcile the demands of unification and constraints imposed by a fit of the fermion data using the GUT specific fermion mass formulae. In order to proceed toward the example fits that prove by construction that such completely realistic fits exist, and for reasons of space, we do not present a survey of RG corrections over the huge parameter space. However certain plots that attempt to illustrate some typical behaviors are given in [25].

\section{NMSGUT derived parameters of the Effective Action}

\subsection{Effective fermion Yukawas and Weinberg Operator coefficients from the NMSGUT}

As in the case of the MSGUT one imposes the fine tuning condition DetH $=0$ to keep a pair of Higgs doublets $H_{(1)}, \bar{H}_{(1)}$ (left and right null eigenstates of the mass matrix $\mathcal{H}$ ) light. The composition of these null eigenstates in terms of the GUT scale doublets then specifies how much the different doublets contribute to the low energy EW scale symmetry breaking. In the Dirac mass matrices we can replace $\left\langle h_{i}>\rightarrow \alpha_{i} v_{u},<\bar{h}_{i}>\rightarrow \bar{\alpha}_{i} v_{d}\right.$ (see Appendix A and [3]). The fermion Dirac masses may be read off the decomposition of $\mathbf{1 6} \cdot \mathbf{1 6} \cdot(\mathbf{1 0} \oplus \mathbf{1 2 0} \oplus \overline{\mathbf{1 2 6}})$ given in [3,6] and this yields (we have made slight changes in notation relative to [16]). The

$$
\begin{aligned}
y^{u} & =(\hat{h}+\hat{f}+\hat{g}) \quad ; \quad \hat{r}_{1}=\frac{\bar{\alpha}_{1}}{\alpha_{1}} ; \quad \hat{r}_{2}=\frac{\bar{\alpha}_{2}}{\alpha_{2}} \\
y^{\nu} & =\left(\hat{h}-3 \hat{f}+\left(\hat{r}_{5}-3\right) \hat{g}\right) \quad ; \quad \hat{r}_{5}=\frac{4 i \sqrt{3} \alpha_{5}}{\alpha_{6}+i \sqrt{3} \alpha_{5}} \\
y^{d} & =\left(\hat{r}_{1} \hat{h}+\hat{r}_{2} \hat{f}+\hat{r}_{6} \hat{g}\right) ; \quad \hat{r}_{6}=\frac{\bar{\alpha}_{6}+i \sqrt{3} \bar{\alpha}_{5}}{\alpha_{6}+i \sqrt{3} \alpha_{5}} \\
y^{l} & =\left(\hat{r}_{1} \hat{h}-3 \hat{r}_{2} \hat{f}+\left(\hat{\bar{r}}_{5}-3 \hat{r}_{6}\right) \hat{g}\right) ; \quad \hat{\bar{r}}_{5}=\frac{4 i \sqrt{3} \bar{\alpha}_{5}}{\alpha_{6}+i \sqrt{3} \alpha_{5}} \\
\hat{g} & =2 i g \sqrt{\frac{2}{3}}\left(\alpha_{6}+i \sqrt{3} \alpha_{5}\right) \quad ; \quad \hat{h}=2 \sqrt{2} h \alpha_{1} ; \hat{f}=-4 \sqrt{\frac{2}{3}} i f \alpha_{2}
\end{aligned}
$$

The Yukawa couplings of matter fields with $\mathbf{1 2 0}$ Higgs field give no contribution to the Majorana mass matrix of the superheavy neutrinos $\bar{\nu}_{A}$ so it remains $M_{A B}^{\bar{\nu}}=8 \sqrt{2} f_{A B} \bar{\sigma}$. Thus the Type I contribution is obtained by eliminating $\bar{\nu}_{A}$

$$
\begin{aligned}
W & =\frac{1}{2} M_{A B}^{\bar{\nu}} \bar{\nu}_{A} \bar{\nu}_{B}+\bar{\nu}_{A} m_{A B}^{\nu} \nu_{B}+\ldots . . \rightarrow \frac{1}{2} M_{A B}^{\nu(I)} \nu_{A} \nu_{B}+\ldots . \\
M_{A B}^{\nu(I)} & =-\left(\left(m^{\nu}\right)^{T}\left(M^{\bar{\nu}}\right)^{-1} m^{\nu}\right)_{A B}
\end{aligned}
$$


As shown in [15, 16] it is likely that the Type II seesaw contribution is subdominant to the Type I seesaw. However the consistency of the assumption that it is negligible must be checked and quantified so we also evaluate the tadpole that gives rise to the Type II seesaw since the 120plet does contribute new terms.

For computing the vev $<\bar{O}\{1,3,1,2\} \overline{126}>$ which gives rise to the Type II seesaw mass, the relevant terms in the superpotential are

$$
\begin{aligned}
W_{F M}^{\bar{\Sigma}}= & M_{O} \vec{O} \cdot \vec{O}-\frac{\bar{\gamma}}{\sqrt{2}} H^{\alpha \dot{\alpha}} \Phi_{44 \dot{\alpha}}^{\beta} \bar{O}_{\alpha \beta}-\frac{\gamma}{\sqrt{2}} H^{\alpha \dot{\alpha}} \Phi_{\dot{\alpha}}^{44 \beta} O_{\alpha \beta} \\
- & 2 \sqrt{2} i \eta\left(\Sigma_{4}^{4 \alpha \dot{\alpha}} \Phi_{44 \dot{\alpha}}^{\beta} \bar{O}_{\alpha \beta}+\bar{\Sigma}_{4}^{4 \alpha \dot{\alpha}} \Phi_{\dot{\alpha}}^{44 \beta} O_{\alpha \beta}\right) \\
+ & \sqrt{2} \zeta\left[\frac{1}{2} \Theta_{\dot{\alpha}}^{\beta} \bar{\Phi}^{44 \alpha \dot{\alpha}}+\Theta_{4}^{4 \alpha \dot{\alpha}} \bar{\Phi}_{\dot{\alpha}}^{44 \beta}\right] O_{\alpha \beta} \\
+ & \bar{\zeta} \sqrt{2}\left[\frac{1}{2} \Theta_{\dot{\alpha}}^{\beta} \Phi_{44}^{\alpha \dot{\alpha}}-\Theta_{4}^{4 \alpha \dot{\alpha}} \Phi_{44 \dot{\alpha}}^{\beta}\right] \bar{O}_{\alpha \beta} \\
= & M_{O} \bar{O}_{-} O_{+}+\bar{O}_{-}\left(i \bar{\gamma} \bar{\alpha}_{1}+2 i \sqrt{3} \eta \bar{\alpha}_{3}+\sqrt{3} \bar{\zeta}_{6}+i \bar{\zeta} \bar{\alpha}_{5}\right) \bar{\alpha}_{4} v_{d}^{2} \sqrt{2} \\
& -O_{+}\left(i \gamma \alpha_{1}+2 i \sqrt{3} \eta \alpha_{2}-\sqrt{3} \zeta \alpha_{6}+i \zeta \alpha_{5}\right) \alpha_{4} v_{u}^{2} \sqrt{2}
\end{aligned}
$$

Here the fields named $O, \bar{O}$ transform as $\{1,3,1, \mp 2\}$ with respect to the LR symmetric gauge group $G_{3221}$ (with $U(1)_{B-L}$ as the Abelian factor) and are the left-handed triplets contained in $(10,3,1),(\overline{10}, 3,1)$ PS sub-representations of $\boldsymbol{\Sigma}(\mathbf{1 2 6}), \overline{\mathbf{\Sigma}}(\overline{\mathbf{1 2 6}})$ respectively. Our phase conventions [3] have for SU(4) 15 plet components $\phi^{A}, A=1 \ldots 15: \phi_{\mu}^{\nu}=\sum_{A=1}^{15} i \phi^{A} \lambda^{A}{ }_{\mu}^{\nu} / \sqrt{2}$. So the electrically neutral component $\bar{O}_{-}$of $\bar{O}\{1,3,1,2\}$ has vev

$$
<\bar{O}_{-}>=\left(i \gamma \sqrt{2} \alpha_{1}+2 i \sqrt{6} \eta \alpha_{2}-\sqrt{6} \zeta \alpha_{6}+i \sqrt{2} \zeta \alpha_{5}\right) \alpha_{4} \frac{v_{u}^{2}}{M_{O}}
$$

and $M_{O}$ can be read off from Table in Appendix $\mathbf{B}$ to be $M_{O}=2(M+\eta(3 a-p))$. The Type II neutrino mass is then simply $M_{A B}^{\nu}=16 i f_{A B}<\bar{O}_{-}>$. However, as found in [15, 16] when computed in the MSGUT these masses are always negligible.

The NMSGUT derived formulae for the matter fermion Yukawas given in this section combined with the explicit formulae [25] for the Higgs fractions $\alpha_{i}, \bar{\alpha}_{i}$, serve as the basis for our investigation of the ability of the NMSGUT to fit all the fermion mass data now available.

As discussed in [51,52] the influence of conventions for phase choices is crucial and and requires painful tracing of equivalences before numerical results for mass spectra can be compared. Comparison with other works [20] that attempt fits with the same Higgs representations but based on generic fermion Yukawa formulae (rather than those specified by the explicit GUT scale symmetry breaking which are used here) has become less interesting since our demonstration that the generic fits are probably unrealizable in the NMSGUT due to the extreme non-linearity of the translation and the hidden and insoluble constraints on fundamental GUT parameters that the generic fits imply 23. Hence we do not discuss this relation further here but refer the interested reader to the previous arXiv versions of this paper if she wishes to find the relations between the parameters. See however our remarks on the magnitudes of parameters used in generic and NMSGUT specific fits in subsection 7.1.

\subsection{Dimension 5 Operators for B,L violation}

In [6] we worked out the effective $d=4$ superpotential for $B+L$ violating processes due to exchange of color triplet superheavy chiral supermultiplets contained in the $\mathbf{1 0}, \overline{\mathbf{1 2 6}}$ Higgs multiplets. These included a novel channel due to decays mediated by exchange of triplets $t_{(4)}\left[3,1, \pm \frac{2}{3}\right]$ contained in the $\overline{\mathbf{1 2 6}}$ Higgs irrep. Evidently the inclusion of $\mathbf{1 2 0}$ plet Higgs will lead to additional channels 
for baryon violation. These can be easily derived using the Pati-Salam decomposition of the 16.16.120 $\mathrm{SO}(10)$ invariants [3] :

$$
\begin{aligned}
\frac{1}{(3 !)} \psi C_{2}^{(5)} \gamma_{i} \gamma_{j} \gamma_{k} \chi \Theta_{i j k}= & -2\left(\bar{\Theta}_{(s)}^{\mu \nu} \psi_{\mu}^{\alpha} \chi_{\nu \alpha}+\Theta_{\mu \nu}^{(s)} \widehat{\psi}^{\mu \dot{\alpha}} \widehat{\chi}_{\dot{\alpha}}^{\nu}\right)-2 \sqrt{2} \Theta_{\nu}^{\mu \alpha \dot{\alpha}}\left(\widehat{\psi}_{\dot{\alpha}}^{\nu} \chi_{\mu \alpha}-\psi_{\mu \alpha} \widehat{\chi}_{\dot{\alpha}}^{\nu}\right) \\
& -2\left(\Theta_{\mu \nu}^{(a)} \dot{\alpha} \dot{\beta} \widehat{\psi}_{\dot{\alpha}}^{\mu} \widehat{\chi}_{\dot{\beta}}^{\nu}+\widetilde{\Theta}_{(a)}^{\mu \nu \alpha \beta} \psi_{\mu \alpha} \chi_{\nu \beta}\right)+\sqrt{2} \Theta^{\alpha \dot{\alpha}}\left(\psi_{\dot{\alpha}}^{\mu} \chi_{\mu \alpha}-\psi_{\mu \alpha} \widehat{\chi}_{\dot{\alpha}}^{\mu}\right) \\
W_{F M}^{\Theta}= & 2 \sqrt{2} g_{A B}\left[\bar{h}_{5}\left(\bar{d}_{A} Q_{B}+\bar{e}_{A} L_{B}\right)-h_{5}\left(\bar{u}_{A} Q_{B}+\bar{\nu}_{A} L_{B}\right)\right] \\
& -2 \sqrt{2} g_{A B}\left[\sqrt{2} \bar{L}_{2} Q_{A} Q_{B}+F_{4} L_{A} L_{B}+\sqrt{2} \bar{t}_{6} Q_{A} L_{B}\right. \\
+ & \left.2 \sqrt{2} L_{2} \bar{u}_{A} \bar{d}_{B}+\sqrt{2} t_{6}\left(\bar{u}_{A} \bar{e}_{B}-\bar{d}_{A} \bar{\nu}_{B}\right)+2 \bar{F}_{4} \bar{\nu}_{A} \bar{e}_{B}\right] \\
& -2 \sqrt{2} g_{A B}\left[2 \bar{C}_{3} \bar{d}_{A} Q_{B}-2 C_{3} \bar{u}_{A} Q_{B}+\frac{i}{\sqrt{3}} \bar{h}_{6}\left(\bar{d}_{A} Q_{B}-3 \bar{e}_{A} L_{B}\right)\right. \\
& -\frac{i}{\sqrt{3}} h_{6}\left(\bar{u}_{A} Q_{B}-3 \bar{\nu}_{A} L_{B}\right)+2 \bar{D}_{3} \bar{e}_{A} Q_{B}-2 \bar{E}_{6} \bar{\nu}_{A} Q_{B} \\
+ & \left.2 E_{6} \bar{d}_{A} L_{B}-2 D_{3} \bar{u}_{A} L_{B}\right]-2 i \sqrt{2} g_{A B}\left[\epsilon \bar{J}_{5} \bar{d}_{A} \bar{d}_{B}\right. \\
+ & 2 K_{2} \bar{d}_{A} \bar{e}_{B}-\epsilon \bar{K}_{2} \bar{u}_{A} \bar{u}_{B}-2 J_{5} \bar{u}_{A} \bar{\nu}_{B} \\
& \left.-\sqrt{2} \epsilon \bar{t}_{7} \bar{d}_{A} \bar{u}_{B}-\sqrt{2} t_{7}\left(\bar{d}_{A} \bar{\nu}_{B}-\bar{e}_{A} \bar{u}_{B}\right)\right]-2 g_{A B}\left[\epsilon P_{2} Q_{A} Q_{B}+2 \bar{P}_{2} Q_{A} L_{B}\right]
\end{aligned}
$$

We have suppressed SM indices and used a sub-multiplet naming convention specified in Appendix B. Conversion to fields of unit norm in the terms containing color sextets $\left(L_{2}, \bar{L}_{2}\right)$ is explained in the caption to the table of unmixed masses in Appendix $\mathbf{B}$.

In order that exchange of a Higgs that couples to matter with a given $B+L$ lead to a $B+L$ violating $d=5$ operator in the effective theory at sub GUT energies it is necessary that it have a non-zero contraction with a conjugate (MSSM) representation Higgs that couples to a matter chiral bilinear with a $B+L$ different from the conjugate of the first $B+L$ value. On inspection one finds that not only the familiar triplet types $\left[\overline{3}, 1, \pm \frac{2}{3}\right] \subset \mathbf{1 2 0}$ i.e $\left\{\bar{t}_{(6)}, \bar{t}_{(7)}\right\}\left[\overline{3}, 1, \frac{2}{3}\right]$ and $\left\{t_{(6)}, t_{(7)}\right\}$ but also novel exchange modes from the $P\left[3,3, \pm \frac{2}{3}\right]$ and $K\left[3,1, \pm \frac{8}{3}\right]$ multiplet types can contribute to baryon violation. In the case of the $\overline{\mathbf{1 2 6}}$ the $\bar{P}_{1}, K_{1} \subset \overline{\mathbf{1 2 6}}$ multiplets did couple to the fermions but $P_{1}, \bar{K}_{1} \subset \mathbf{1 2 6}$ did not. The $\mathbf{1 2 0}$ however contains both $P_{2}, \bar{P}_{2}$ and $K_{2}, \bar{K}_{2}$. Since these mix with $P_{1}, \bar{P}_{1}$ and $K_{1}, \bar{K}_{1}$, a number of fresh contributions appear.

Note in particular that these novel exchanges always lead to contributions in which at least one and possibly both pairs of final state family indices are antisymmetrized. On integrating out the heavy triplet Higgs supermultiplets one obtains the following effective $d=4$ superpotential for baryon Number violating processes in the NMSGUT to leading order in $m_{W} / M_{X}$ :

$$
W_{e f f}^{\Delta B \neq 0}=-L_{A B C D}\left(\frac{1}{2} \epsilon Q_{A} Q_{B} Q_{C} L_{D}\right)-R_{A B C D}\left(\epsilon \bar{e}_{A} \bar{u}_{B} \bar{u}_{C} \bar{d}_{D}\right)
$$

where the coefficients are

$$
\begin{aligned}
L_{A B C D} & =\mathcal{S}_{1}{ }^{1} \tilde{h}_{A B} \tilde{h}_{C D}+\mathcal{S}_{1}{ }^{2} \tilde{h}_{A B} \tilde{f}_{C D}+\mathcal{S}_{2}{ }^{1} \tilde{f}_{A B} \tilde{h}_{C D}+\mathcal{S}_{2}{ }^{2} \tilde{f}_{A B} \tilde{f}_{C D} \\
& -\mathcal{S}_{1}{ }^{6} \tilde{h}_{A B} \tilde{g}_{C D}-\mathcal{S}_{2}{ }^{6} \tilde{f}_{A B} \tilde{g}_{C D}+\sqrt{2}\left(\mathcal{P}^{-1}\right)_{2}{ }^{1} \tilde{g}_{A C} \tilde{f}_{B D} \\
& -\left(\mathcal{P}^{-1}\right)_{2}{ }^{2} \tilde{g}_{A C} \tilde{g}_{B D}
\end{aligned}
$$


and

$$
\begin{aligned}
R_{A B C D} & =\mathcal{S}_{1}{ }^{1} \tilde{h}_{A B} \tilde{h}_{C D}-\mathcal{S}_{1}{ }^{2} \tilde{h}_{A B} \tilde{f}_{C D}-\mathcal{S}_{2}{ }^{1} \tilde{f}_{A B} \tilde{h}_{C D}+\mathcal{S}_{2}{ }^{2} \tilde{f}_{A B} \tilde{f}_{C D} \\
& -i \sqrt{2} \mathcal{S}_{4}{ }^{1} \tilde{f}_{A B} \tilde{h}_{C D}+i \sqrt{2} \mathcal{S}_{4}{ }^{2} \tilde{f}_{A B} \tilde{f}_{C D} \\
& +\mathcal{S}_{6}{ }^{1} \tilde{g}_{A B} \tilde{h}_{C D}-i \mathcal{S}_{7}{ }^{1} \tilde{g}_{A B} \tilde{h}_{C D}-\mathcal{S}_{6}{ }^{2} \tilde{g}_{A B} \tilde{f}_{C D}+i \mathcal{S}_{7}{ }^{2} \tilde{g}_{A B} \tilde{f}_{C D} \\
& +i \mathcal{S}_{1}{ }^{7} \tilde{h}_{A B} \tilde{g}_{C D}-i \mathcal{S}_{2}{ }^{7} \tilde{f}_{A B} \tilde{g}_{C D}+\sqrt{2} \mathcal{S}_{4}{ }^{7} \tilde{f}_{A B} \tilde{g}_{C D} \\
& +i \mathcal{S}_{6}{ }^{7} \tilde{g}_{A B} \tilde{g}_{C D}+\mathcal{S}_{7}{ }^{7} \tilde{g}_{A B} \tilde{g}_{C D}-\sqrt{2}\left(\mathcal{K}^{-1}\right)_{1}{ }^{2} \tilde{f}_{A D} \tilde{g}_{B C} \\
& -\left(\mathcal{K}^{-1}\right)_{2}{ }^{2} \tilde{g}_{A D} \tilde{g}_{B C}
\end{aligned}
$$

here $\mathcal{S}=\mathcal{T}^{-1}$ and $\mathcal{T}$ is the mass matrix for $[3,1, \pm 2 / 3]$-sector triplets : $W=\bar{t}^{i} \mathcal{T}_{i}^{j} t_{j}+\ldots$, while

$$
\tilde{h}_{A B}=2 \sqrt{2} h_{A B} \quad \tilde{f}_{A B}=4 \sqrt{2} f_{A B} \quad \tilde{g}_{A B}=4 g_{A B}
$$

These operators are dressed by sparticles to yield the $d=6$ effective 4 -fermi operators for baryon decay. This dressing requires knowledge of the sparticle spectra and mixing angles. This information is determined via the invocation of threshold corrections used to fit the down and strange quark masses which assume adequate(diagonal)sfermion spectra for the purpose. However the scalar mixing which is so crucial to the baryon decay rate is assumed minimal i.e to be determined simply by evolution of the GUT scale (super)CKM mixing. The rates for B violation via the dominant $d=5$ operators are evaluated using the above formulae and the usual dressing by Gaugino/Higgsino exchange.

\section{Method for Numerical searches}

We now describe our numerical procedures and application of Susy threshold corrections. We use the 2-loop RG equations for the MSSM [31,53] to extrapolate the central values of fermion Yukawa data at $M_{Z}$ (including neutrino mass splitting and mixing data, see [55/57] for recent values), up to the MSSM one-loop gauge unification scale $M_{X}^{0}=10^{16.33} \mathrm{GeV}$ while ignoring, for the time being, complications such as right-handed neutrino and supersymmetric thresholds. In the case of the neutrino masses(Type I seesaw) we extrapolate the coefficient 32] $\kappa_{A B}=M_{\nu} /\left(2 v_{u}^{2}\right)$ of the $S U(2) \times U(1)$ invariant dimension 5 operator $[45,58$, that gives rise to neutrino mass when the electroweak vev $\left(v_{u}=174 \sin \beta \mathrm{GeV}\right.$; for the $\mathrm{SM} \sin \beta$ is 1$)$ is substituted for the two electroweak doublets in it. We assume that the neutrino mass hierarchy is normal and that the lightest neutrino species has a very small mass $<1 \mathrm{meV}$.

After two-loop RG evolution [31] up-to $M_{X}^{0}$ we re-extract canonical input parameters i.e the 9 charged fermion Yukawas, the 4 CKM parameters, and the 5 neutrino mass data. We use percentage uncertainties in fermion Yukawas at $M_{X}^{0}=10^{16.33} \mathrm{GeV}$ recently re-estimated in [30], which include the large uncertainties $\left(\delta O_{i}\right)$ due to large threshold effects on down type quarks and charged leptons at large $\tan \beta$. We form $\chi^{2}=\sum_{i}\left(\left(O_{i}-\bar{O}_{i}\right) / \delta O_{i}\right)^{2}$ which compares the central values $\left(\bar{O}_{i}\right)$ with the values of the same parameters $\left(O_{i}\right)$ calculated using the formulae described in Section $\mathbf{5}$ and normalized by the errors $\left(\delta O_{i}\right)$. The 'down hill simplex' or "amoeba" algorithm of Nelder and Mead [59] is then used to randomly troll the 38 dimensional (real) hyperspace of NMSGUT parameters.

After diagonalization of the 10-plet Yukawas using the $U(3)$ generation basis freedom with respect to the matter 16-plets and after using the rephasing freedom for Higgs fields, the parameters consist of (numbers in brackets denote the number of real parameters) $\left\{h_{i i}(3), f_{i j}(12), g_{i j}(6)\right.$, $\eta(2), \rho(2), k(2), \gamma(1), \bar{\gamma}(1), \zeta(2), \bar{\zeta}(2)\}$ which are 33 in number and an additional $5\left\{m(1), \tilde{m_{\Theta}}(1)\right.$, $|\lambda|(1), \xi \Leftarrow x(2)\} . M_{H}$ has been fixed by fine-tuning the doublet masses. The randomly chosen complex parameter $x$, which is central to the explicit solution of the GUT symmetry breaking 
problem, is used to generate $\xi=\frac{\lambda M}{\eta m}$ (using( A.8) ). $\xi$ stands in for the real parameter M and hence the phase of $\lambda$ is determined to be $\operatorname{Arg}(\xi)+\operatorname{Arg}(\eta)$ for consistency. Note that with the rescaled form $\tilde{m}_{\Theta}=m_{\Theta} \lambda / m$ chosen to be real the value of $m_{\Theta}$ will have the phase of $\lambda^{*}$ : as seen in the explicit fits given in Appendix D. While searching we imposed penalties to keep all the Yukawas and GUT superpotential parameters within the perturbative range and the threshold corrected unification parameters [3, 16] $\Delta_{X}=\Delta \log _{10} M_{X}, \Delta_{G}=\Delta \alpha_{G}^{-1}\left(M_{X}\right), \Delta_{3}=\Delta \hat{\alpha}_{3}\left(M_{Z}\right)$ within reasonable limits given in eqn(4.5). It is worth emphasizing that the single superheavy mass scale $m$ (the $\mathbf{2 1 0}$ mass parameter in the superpotential) of the NMSGUT, which controls both baryon violation and neutrino masses, is determined by the RG analysis that fixes $\Delta_{X}$ [16]. Even though the vev of the $\overline{\mathbf{1 2 6}}$ is $O\left(M_{X}\right)$ it's small couplings confer a freedom to lower right-handed neutrino masses and thus raise the light neutrino masses to acceptable values.

The programs were written in FORTRAN95 and run on PC's with a random starting point. Currently the code runs to a few tens thousands of lines including borrowed subroutines, mainly from SPHENO [64]. The solutions found on the first run (before applying the Susy threshold corrections to lower $y_{d, s}^{M S S M}$ ) present the following salient features :

- Neutrino masses and large mixing angles were accurately fitted.

- The values of the $\overline{\mathbf{1 2 6}}$ couplings were so tiny that the three right-handed neutrino masses were much lighter than $M_{X}$ and spanned the range $\sim 10^{8}-10^{13} \mathrm{GeV}$, just as conjectured by us earlier [16, 43, 44]. Note that this is the range required by most (Type I) leptogenesis models.

- The bulk of the residual $\chi^{2}$ typically consisted of errors in the d,s quark Yukawa couplings.

- The right-handed neutrino mass scale was not independently chosen and then checked for approximate consistency(as in an early version of this work [37]) but taken to be the exact expression dictated by the NMSGUT vevs.

Having found solutions acceptable except for the small down and strange quark Yukawas, we extrapolate the fermion Yukawas (neglecting generation mixing for simplicity) and randomly chosen Supergravity(SUGRY) type supersymmetry breaking parameters with non-universal Higgs $\operatorname{masses}(\mathrm{NUHM})$ at $M_{X}^{0}\left(M_{\text {gaugino }}=m_{1 / 2}, m_{\tilde{f}}=m_{0}, A^{0}, m_{H_{u}}^{2}, m_{H_{d}}^{2}\right)$ back down to $M_{Z}$ and use the diagonal Yukawa couplings as targets of a downhill simplex fitting procedure that incorporates the large $\tan \beta$ Susy threshold corrections. We use the formulae given in 60 to estimate the leading (gluino and chargino diagram) corrections to the charged fermion masses but slightly modify them using [30,61] to also include Bino corrections. We also crosschecked that the corrections to the $T_{3 L}=-1 / 2$ fermion masses obtained therefrom are equal within a few percent to the large $\tan \beta$ corrections calculated using the formulae of [61] which are in the limit of unbroken electroweak symmetry. Although the threshold corrections to the up and charm quark Yukawas(calculated using the formulae of [60]) are very small the top quark mass suffers threshold corrections $10-15 \%$ which must be included. Once a set of SUGRY-NUHM parameters (at $M_{X}^{0}$ ) which gives corrected MSSM values reasonably close to the target values is found we extrapolate the corrected values back up to $M_{X}^{0}$ and start a fresh search(starting from the last achieved values of the GUT scale parameters) for a new fit to the complete set of fermion Yukawa parameters. Since the values of the threshold corrected down and strange quark Yukawas are now much smaller due to the Susy threshold corrections invoked to fit the small values achieved in the earlier cycle, much lower values of $\chi^{2}$ can now be achieved. However it is still much easier to find fits in which $\sin ^{2} \theta_{12}$ or $\sin ^{2} \theta_{23}$ is very small than to find fits with both leptonic mixing angles are small. In fact the false minima in $\chi_{X}^{2}$ due to this type of solution are a trap the search program falls into 
repeatedly. It is amusing to note that some years ago this could have been heard as a vox ex machina predicting the small mixing angle solution of the solar neutrino problem ! Iterations of this procedure can give fairly accurate fits in which the 18 data values at the high scale $\left(M_{X}^{0}\right)$ are fit with a total $\chi_{X}<10^{-1}$. Also at the scale $M_{Z}$ one may achieve $\left(\chi_{Z}<10^{-1}\right)$. A tension between the threshold corrected values of $y_{b}\left(M_{Z}\right)$ which are lower than the values required by the fit using NMSGUT formulae is often noted (see e.g. [62]) when $A_{0}, \mu$ are small and there are thus only small threshold corrections. This is even used to argue for negative values of $\mu$. However since we have allowed quite a large little hierarchy and large $A_{0}$ values we can in fact operate with close to central values $\left(m_{b}\left(M_{Z}\right)^{S M}=2.9 \pm 0.1 \mathrm{GeV}\right)$ as also for all the other SM and neutrino oscillation parameters. In this sense the region of Susy breaking soft parameters we focus on seems quite distinct and novel compared to most earlier studies.

\section{Examples of Soft parameters sets and Susy Spectra found}

\subsection{Assumptions and generic features}

Recently preliminary results from the $1 \mathrm{fb}^{-1}$ data accumulation at LHC has been announced [34] and allowed a ( $\tan \beta$ independent) inference that colored sparticles masses are higher than about $500 \mathrm{GeV}$ and charged exotics like $\tilde{l}, \tilde{W}^{ \pm}$more massive than about $200 \mathrm{GeV}$. When searching for the soft parameters which would cure the too low d-s-b quark Yukawa limitation 20] of the NMSGUT Yukawa formulae we imposed the following constraints on the soft supersymmetry breaking parameters [63] :

$$
\begin{aligned}
& \left|\tilde{m}_{\bar{l}, L} / M_{1}\right| \geq 0.9 \quad ; \quad\left|\tilde{m}_{\bar{q}, Q} / M_{3}\right| \geq 0.75 \\
& \mu,\left|A_{0}\right|<150 \mathrm{TeV} \quad ; \quad m_{\tilde{l}, H^{ \pm}}>200 \mathrm{GeV} \\
& \tilde{W}: 200 \mathrm{GeV}<M_{2}\left(M_{Z}\right)<1000.0 \mathrm{GeV} \\
& \tilde{g}: 500 \mathrm{GeV}<M_{3}\left(M_{Z}\right)<1000.0 \mathrm{GeV} \quad ; \quad m_{\tilde{q}}>500 \mathrm{GeV}
\end{aligned}
$$

We also required the SM Higgs to be heavier than $114 \mathrm{GeV}$ and the Bino lighter than the lightest sfermion.

In Tables 3-14 in Appendix D we present the NMSGUT-SUGRY-NUHM parameters at $M_{X}^{0}=$ $10^{16.33} \mathrm{GeV}$ of two fairly precise fits to the complete fermion data. In Table 3 ( Table 9) we give NMSGUT superpotential parameters, superheavy mass spectra, threshold corrected gaugeunification parameters, soft susy breaking seed parameters and the strength of B-violation at $M_{X}^{0}$ for the first (second) fit. In Table 4 (Table 10) we give the Target values, uncertainties and achieved values of the yukawa couplings at $M_{X}^{0}$ along with the Pulls for the first (second) fit. In Table 5 (Table 11) we give NMSGUT derived fermion masses at $M_{Z}$ before and after Susy threshold corrections for the first (second) fit. In Table 6 (Table 12) we give the values of the soft supersymmetry breaking parameters at $M_{Z}$ that arise after two loop RG evolution(with no mixing) from $M_{Z}$ for the first (second) fit. In Tables 7,8 (Tables 13,14) we give the sparticle spectra calculated with and without the use of generation mixing in the RG flow for the first (second) fit. We see that the 18 observables are fitted rather well with minimal tension visible even in the troublesome $T_{3}=-\frac{1}{2}$ Yukawa couplings. However this is done only as a proof of principle and feasibility and as an example of the typical form of non pathological exemplars of our fitting program. It would be highly premature to subscribe to any one parameter set as specifically demanded by NMSGUT fits.

In the type of mass spectra we encounter here, the LSP (stable due to the primary virtue of R-parity preservation down to low energies [1,2]) is pure Bino. Surprisingly it (often) happens that the smuon emerges lighter than the Bino and this needs to be avoided by a penalty in the 
search to avoid a charged stable LSP. It is interesting that a tendency to such a pairing of a Bino LSP and light smuon may make the Bino an effective dark matter candidate by providing an effective co-annihilation channel for Bino Cold Dark Matter. This tendency is visible in both our example fits(see Tables 7 and 13) where the (right) smuon is lighter than the selectron and thus closest to the light Bino LSP. We shall return to the cosmology of these models elsewhere. On the other hand, since we are still to implement loop corrections to scalar masses (which may be very significant for light sparticles) it would be wise to hold enthusiasm for a particular low tree level slepton mass in check till it survives loop corrections.

The conventional wisdom 63] that the sfermions of the third generation will be much lighter than the first two generations of squarks due to strong renormalization of the common sfermion mass at $M_{X}^{0}$ by top Yukawa driven effects is directly challenged by our spectra which yield third generation sfermions much heavier than the first two generations. This feature marks out such sfermion spectra as linked to the inner logic governing the fermion mass pattern in the NMSGUT and we proffer it as a prediction regarding supersymmetric physics that may be tested by LHC or its successors. The large value of the third generation scalar trilinear may also become relevant phenomenologically since it leads to large L-R mixing in the sfermion sector for the third generation and thus to enhancement of various processes suppressed by the low value of $M_{L R}^{2}$ in many scenarios.

The structure of the Susy threshold corrections and the electroweak symmetry breaking conditions which determine $|\mu|, B$ in terms of $m_{H, \bar{H}}^{2}, \tan \beta$ are reviewed in detail in Appendix C. From the discussion there it emerges that the NMSGUT with viable Type I seesaw masses is inescapably linked to third generation squarks more massive than the first two generation squarks, large negative values of the trilinear scalar parameters and also a large $\mu$ parameter. Although such distinctive requirements on the soft sector are welcome we should recognize that they do imply a considerable degree of fine tuning to assure viable electroweak symmetry breaking : as will be visible in the small value of the ratio $M_{W} / \mu \sim 10^{-3}$. However such a fine tuning is generally needed by large $\tan \beta$ models. In contrast with the first version of this paper(where we searched the soft Susy parameter space at $M_{S}$ ) we have now achieved fits to the required low values of $y_{d, s}$ using constrained SUGRY-NUHM Susy breaking where the 5 element (i.e $\left.m_{1 / 2}=M_{\text {gaugino }}, m_{\tilde{f}}=m_{16}, A_{0}, M_{H, \bar{H}}^{2}\right)$ parameter sets are specified at $M_{X}^{0}$. These 5 parameters are chosen directly at $M_{X}^{0}$ by the random search while the parameters $\mu\left(M_{X}^{0}\right), B\left(M_{X}^{0}\right)$ are fixed by first imposing the one-loop corrected EWRSB conditions at $M_{Z}$ (calculated using a subroutine taken from the program SPHENO [64] $)$ to fix $\mu\left(M_{Z}\right), B\left(M_{Z}\right)$ to ensure acceptable electroweak symmetry breaking with the given $\tan \beta$ and vacuum expectation values and then running these back up to $M_{X}^{0}$. Notice that the freedom to do this comes from the non-dependence of the RG flows of the rest of the couplings and masses, on the $\mu, B \mathrm{RG}$ flows. This is a crucial enabling feature of RG flows in Susy GUT scenarios. The non-universal Higgs mass parameters used are justifiable even in the Grand Unified context since the light Higgs doublets arise as a mixture of 6 doublets from 5 different GUT Higgs multiplets namely the $\mathbf{1 0}, \mathbf{1 2 0}, \mathbf{1 2 6}, \overline{\mathbf{1 2 6}}, \mathbf{2 1 0}$. The GUT symmetry justifies assuming distinct soft masses for each representation and the light doublets are linear combinations of Higgs doublets from 5 sources in the GUT (with coefficients determined by the unitary matrices that diagonalize the doublet mass matrices). Thus freedom to choose two non-universal Higgs masses is certainly conceivable. We also note that the large $\mu, A_{0}$ parameters help avoid problems with FCNC and CCB/UFB instability [38].

\subsection{Dissecting Specimens}

The basic difficulty in building a grand unified theory of fermion masses is the achievement of sufficiently large neutrino masses and the reconciliation between small CKM mixing angles and 
large PMNS ones. So it is interesting to dissect the solutions we have found to see how exactly they circumvent the difficulties typically encountered [15, 16, 43, 44. The roles played in the effective matter fermion Yukawa couplings $Y^{u, d, e}$ and the Weinberg $d=5$ neutrino mass operator coefficients $\kappa_{A B}$ by the Yukawa couplings $h_{A B}, g_{A B}, f_{A B}$ of the three FM Higgs representations are, so to speak, the vital organic functions of the NMSGUT and their dissection reveals the integral unity and 'intelligent design' within the fermion mass pattern. By killing the Yukawa couplings individually or in groups and noting the resultant effect one uncovers their roles.

The most influential coupling is $h_{33}$ which directly affects every mass and mixing angle and may be called the lynch pin of the Yukawa coupling pattern [43,44]. The third generation masses can be fit to within $5 \%$ or better by this coupling alone : this is the content of ' $b-\tau-t$ unification' in this context. The next most important ( and sometimes largest) coupling is $g_{23}$. It is crucial for the $\theta_{23}^{(q)}$ mixing and thus also for second generation masses: specially the muon mass. The importance of the couplings $f_{A B}$ arises from their smallness : since they control the mass and couplings of the superheavy right-handed neutrinos which leave behind a characteristic signature in the Type I seesaw masses (where they enter in the denominator). The limit $f_{A B} \rightarrow 0$ is singular i.e. decreasing $f_{A B}$ changes the effective theory (MSSM with massive left-handed neutrinos) drastically. The couplings $h_{11}, h_{22}, g_{12}, g_{13}$ are relevant for the masses and mixing of the first and second generations.

If we first set $f_{A B}=0$ then all neutrino masses and mixings become unphysical. However one finds that the fractional changes in the charged fermion masses are typically less than one part in $10^{4}$. Thus the $\overline{\mathbf{1 2 6}}$ coupling is irrelevant to the charged fermion sector in our fits. This feature together with much smaller values of the parameter $r_{D} \sim 10^{2}$ (as compared to $r_{D} \sim 10^{5}$ ; see 20,21] for the definition and values of $r_{D}$ which controls the strength of the contribution of $g_{A B}$ to the neutrino Yukawa coupling) marks out the solutions we have found as distinct from the class of generic fits explored in [20,21]. Moreover our $f_{A B}$ eigenvalues (and thus the right-handed neutrino masses) are strongly hierarchical $\left(1: 10^{2}: 10^{4}\right):$ so much so that they are effective in compensating the large hierarchy in the neutrino Dirac Yukawas $\left(10^{-1}: 10: 10^{2}\right)$ (which is amplified by the square of the neutrino Dirac Yukawa in the Type I formula) to produce the rather weak hierarchy of the light neutrino masses. This is again very different from the generic $\mathrm{SO}(10)$ solutions of [20,21] which have a hierarchy of at most $1: 60: 300$ for the right neutrino masses (which are also much larger). There [20,21] the dilution of the Dirac mass hierarchy relies on a strong boost of the coefficient $r_{D}$ of $g_{A B}$ in the neutrino Yukawa matrix to huge values $\sim 10^{4}-10^{5}$. Our parameters simply never venture towards such large $r_{D}$ : they always produce values of the coefficient $r_{D}$ below 500. In fact even if one removes constraints that the parameters remain perturbative it proves extremely difficult to force the downhill simplex 'amoeba' to crawl towards the high $r_{D}$ region. Of course we have already shown [23] that generic numeric fits carry no direct implication for the NMSGUT since their parameters are constrained, but still the generic analysis propagated a scare $[20$ that the small $\overline{\mathbf{1 2 6}}$ scenario was basically flawed and then compounded it by the confusing conclusion that small(but not tiny) $f_{A B}$ solutions were in fact capable of finding accurate fits(by using values of a Yukawa formula parameter $r_{D} \sim 10^{5}$ that are so large as to make them unachievable in actual NMSGUT Yukawa formulae). In any case this history underlines once again [15, 16] the emphatic non sequituur with which explicit UV theories must confront such generic no-go claims unless they are founded on something more than numeric projections.

In [43, 44 we argued that the 2-3 sector should be regarded as the core of the fermion mass hierarchy and that the complete hierarchy could and should be understood semi-analytically by perturbing around the couplings $h_{33}, g_{23}$ i.e by taking these couplings as $O(1)$ and the others as order $O\left(\epsilon^{n}\right), \epsilon^{2} \sim \theta_{23}^{q}$. The solutions we have found by random search bear this out. We could now pursue our earlier proposal [43, 44] to understand the fermion parameters by an expansion 
around the 23 sector in a single hierarchy parameter with confidence that the solutions exist and have the required form. The phases and coefficient values we have found can be checked for their compatibility with such an expansion and thus a qualitative understanding of the hierarchy arrived at. Of course we must keep in mind that the values of the Yukawa couplings we find match the SM precision couplings extrapolated to $M_{X}^{0}$ only after applying the large corrections to $y_{d, s}$ at $M_{S}$ due to large $\tan \beta$ that underpin of our fits. Thus the fermion masses explained above are themselves significantly different from the ones that we earlier [43, 44] had only partial success in describing via the $\epsilon$-expansion. We anticipate that the downwards revision of the target $y_{d}, y_{s}$ in the fit at $M_{X}^{0}$ by a factor of $\epsilon$ will allow the determination of fits with two large angles in the lepton sector, which we could not achieve earlier. As mentioned that the most frequent "false minimae" encountered have almost perfect charged fermion fits but one large and one small leptonic mixing angle. Analytic (perturbative) confirmation of this proposal may be of some value since it may yield a more robust understanding of the innards of the hierarchy.

It is worth mentioning that our perturbative analysis of both the two and three generation (real) tree level fermion Yukawa fitting problem in $\mathrm{SO}(10)$ models based upon a 10-120 FM Higgs system led to the consistency constraint that $\left|\frac{y_{b}-y_{\tau}}{y_{s}-y_{\mu}}\right|=1+O\left(\epsilon^{2}\right)$. It is striking that this constraint is obeyed in the fits without GUT scale threshold corrections that we have found so far even though the parameters are no longer real. Thus the NMSGUT seems constrained to obey the stringent form of $b-\tau$ unification found in [43, 44] so long as GUT scale threshold corrections are unimportant. Conversely, as we show in [38, the inclusion of GUT threshold corrections to fermion Yukawas loosens this constraint and permits one to find solutions that are also B decay realistic. However if one-loop GUT scale threshold corrections to Yukawas 38] are strong then the tree level arguments of the previous paragraph become moot.

\subsection{Nucleon decay rates}

Viable RG flows in the NMSGUT can raise $M_{X}$ by to near the Planck scale (as in the second example in Appendix $\mathbf{D}$ ). Thus $d=6$ (i.e gauge boson mediated) proton decay can be suppressed by up to 8 orders of magnitude relative to the already long life times $\sim 10^{36}$ yrs corresponding to the one-loop unification scale. The question of the rate of $d=5$ operator mediated baryon decay in the theory at hand is clearly the critical one. Since the decay rates in these channels will depend on the soft scalar spectrum and mixing they could provide a welcome additional filtration of the parameter sets that pass the other criteria.

A complete calculation of B-violation rates in all possible channels is so onerous that we would be justified in presenting it only after we have optimized our fits and have otherwise viable candidates. As mentioned above threshold corrections due to 120-plet at $M_{X}$ are important in this theory [38]. Moreover the searches for fits will themselves need to be done keeping in mind the need to lower the B violation rates below the $10^{-28} \mathrm{yr}^{-1}$ that are generically found (see below). Since $d=5$ operators and among them the $p \rightarrow M^{+} \nu$ channels are known to be the dominant modes of $\mathrm{B}$ violation we give the rate of these reactions using the (hard and soft) parameter values found by us. We emphasize that sfermion mixing at $M_{Z}$ is assumed to be driven entirely by that present in the matter Yukawa couplings of the $\mathrm{SO}(10)$ superpotential at $M_{X}^{0}$ (after renormalization down to $M_{Z}$ ). Corrections due to renormalization between $M_{X}$ or $M_{\text {Planck }}$ and $M_{X}^{0}$ are ignored. The technology for this calculation is well known and we have used the calculations of [40, 41] as starting points and cross checks for developing a Mathematica program that calculates the decay rates given the fundamental GUT and SUGRY-NUHM parameters (i.e. Tables 3-14).

The (162 complex ) operator coefficients for B-decay $\left(L_{A B C D}, R_{A B C D}\right)$ need to be renormalized down from the GUT scale to $M_{Z}$ together with the other hard and soft parameters and plugged into the formulae of [41] after appropriately removing the assumptions of an SU(5) GUT context 


\begin{tabular}{|c|c|c|c|c|c|}
\hline Fit & $\tau_{p}\left(M^{+} \nu\right)$ & $\Gamma\left(p \rightarrow \pi^{+} \nu\right)$ & $B R\left(p \rightarrow \pi^{+} \nu_{e, \mu, \tau}\right)$ & $\Gamma\left(p \rightarrow K^{+} \nu\right)$ & $B R\left(p \rightarrow K^{+} \nu_{e, \mu, \tau}\right)$ \\
\hline Ex.1 & $8.1 \times 10^{28}$ & $3.1 \times 10^{-30}$ & $\left\{2.6 \times 10^{-5}, 0.09,0.91\right\}$ & $9.2 \times 10^{-30}$ & $\left\{1.1 \times 10^{-4}, 0.27,0.73\right\}$ \\
Ex.2 & $1.7 \times 10^{28}$ & $7.2 \times 10^{-30}$ & $\left\{3.04 \times 10^{-5}, 0.014,0.986\right\}$ & $5.2 \times 10^{-29}$ & $\left\{5.45 \times 10^{-5}, 0.01,0.99\right\}$ \\
\hline
\end{tabular}

Table 1: Table of $d=5$ operator mediated proton lifetimes $\tau_{p}(\mathrm{yrs})$, decay rates $\Gamma\left(y r^{-1}\right)$ and branching ratios in the dominant Meson $^{+}+\nu$ channels.

and negligible right-handed mixing. The formulae of [40] can be used as a cross check of the calculation. We find the values given in Table 1 .

Clearly these proton decay rates are too large given the current limits $\sim 10^{-33} \mathrm{yr}^{-1}[66$ ]. Thus the otherwise promising fits imply an acute crisis of volatile baryon number. In the next paper of this series [38] we include also GUT scale threshold effects and conduct searches that are optimized with respect to baryon stability. This permits us to find much more realistic fits.

\section{Discussion and Outlook}

In this paper, motivated by successful fits of the fermion data 21, 44 which evade the difficulties that forced an abandonment [15, 16] of the hope [14] that the $\mathbf{1 0}, \overline{\mathbf{1 2 6}}$ FM Higgs system would be sufficient to describe the entire fermion mass spectrum, we specified the ingredients of a New Minimal Supersymmteric GUT based on the gauge group $S O(10)$ and the $\mathbf{2 1 0} \oplus \mathbf{1 0} \oplus \mathbf{1 2 0} \oplus \mathbf{1 2 6} \oplus \overline{\mathbf{1 2 6}}$ Higgs System. While inheriting the Higgs system responsible for GUT scale symmetry breaking unchanged from the MSGUT [10 12] but reassigning the roles of the FM Higgs the NMSGUT is able to describe all the fermion data at $M_{X}$ successfully provided recourse is had to relevant threshold corrections at the Susy breaking scale. This alleviates a problem with fitting down type Yukawa couplings using only the 10,120 couplings to matter fields (since the $\overline{\mathbf{1 2 6}}$ couplings are lowered drastically to make the Type I seesaw neutrino masses viable and are thus irrelevant to charged fermion masses ).

Using the techniques we developed for the MSGUT [3, 6] we computed the superheavy spectrum for the NMSGUT and used it to evaluate threshold effects in the gauge evolution. Our experience with fits is that the Unification scale is generally raised above the one-loop values. We have also attempted surveys to find viable unification parameters $\left(\Delta_{X, G, 3}\right)$ over the (vast) parameter space and these also support this intuition [24,25]. This increase can take $M_{X}$ to values as large as $M_{\text {Planck }}$ while still remaining in the perturbative domain. Thus gauge mediated baryon decay is unmeasurably small in this theory. Together with $M_{X}$ all other masses, in particular those of the three triplet types that mediate $d=5$ baryon decay, also rise. Thus, prima facie, not only $d=6$ but also $d=5$ proton decay may be controllable. However when we searched for fits without imposing any constraint on B-violation rates the rates are faster than the current limits by about 6 orders of magnitude. We find [38] that inclusion of GUT scale threshold effects due to the 120-plet and searches of the parameter space under a constraint to suppress B-violation is necessary before acceptable B-violation rates are reached.

It should be kept in mind that our results carry systematic errors due to the omission of various effects such as right-handed neutrino thresholds (important due to their light masses $\sim 10^{8}-$ $10^{12} \mathrm{GeV}$ ), mass splitting among Susy partners at $M_{Z}$, non-diagonality in the supersymmetric threshold corrections, loop corrections to scalar masses and neglect of renormalization effects from $M_{\text {Planck }}$ (where the soft SUGRY-NUHM parameters should presumably be specified) to $M_{X}^{0}$ which is the high energy matching scale we have chosen for convenience. Accounting for multiple and disparate Susy threshold corrections also leads to errors that are quite complex to account for and 
can be an important source of theoretical uncertainty. The non-diagonal threshold effects at large $\tan \beta$ can be calculated from the same diagrams by inserting the appropriate flavor structure and diagonalization. All these improvements are required for later stages of our program. However these effects are higher order corrections to the large diagonal modifications to $y_{d, s}$ and the cancellation that prevents the bottom Yukawa from also being strongly renormalized by gluino exchange. The non-diagonal effects in both the fermionic and scalar sectors are driven by the small off diagonal Yukawa couplings at $M_{X}$ that we determined by fitting the quark and lepton mixings. Hence they will modify the diagonal effects only marginally. Nevertheless their presence makes it clear that one needs to be circumspect in claiming too high accuracies.

An increase in $M_{X}$ could provide resolution of a nagging difficulty [67] in the MSGUT : the Landau pole in the gauge coupling evolution above $M_{X}$. When $M_{X}$ is closer to the Planck scale the coincidence of the $\mathrm{SO}(10)$ Landau pole with the Planck scale strengthens our speculation that the UV condensation to be expected in such a supersymmetric Asymptotically Strong(AS) theory [67,68] acts as a physical cutoff for the perturbative $\mathrm{SO}(10)$ theory and perhaps even as the scale of an induced gravity that arises from this theory(supersymmetry cures the ambiguities that plagued the original induced gravity ideas [69]). We made a beginning in [68, by demonstrating, using supersymmetric strong coupling heuristics [70], that in a toy AS Susy GUT the condensation actually takes place and breaks the (toy) GUT symmetry, and that the vevs responsible are calculable. It is encouraging that the development of the theory in regard to apparently unrelated features has naturally brought us to the point where a number of intractable fundamental features have become pliable to a synthetic interpretation.

In this paper we have indicated a feasible route to pursue if the New or Full Minimal Supersymmetric $\mathrm{SO}(10)$ GUT is to become vulnerable to falsification by the upcoming data from the LHC and it's successors and the large water Cerenkov detectors now being planned to further raise the limits on proton decay. This route evades difficulties and confusion that had waylaid the fermion fitting program in MSGUTs in the recent past. We have shown that the way out is to accept the difficulties as indicative of a particular type of sfermion spectra which will modify the $d, s$ quark Yukawa couplings significantly at the threshold $M_{S}$. To our knowledge this is a novel proposal that ties the fermion spectrum achievable by a Susy GUT tightly to its soft breaking terms. Such a program is feasible and plausible precisely because of the constraints under which the NMSGUT functions and the lack of freedom to add additional fermion mass operators so abundantly available in $\mathrm{SO}(10)$ Susy theories based on low dimensional Higgs fields and non-renormalizable operators.

If the colliders do not belie their promises they may sketch the supersymmetric spectra for us. The detection of even a single supersymmetric particle(likely a chargino or a smuon in terms of the typical SUGRY-NUHM NMSGUT spectra we find) will anchor the Susy mass patterns emerging from the RG analysis of the NMSGUT. With these spectra in hand it will have to face both edges of the scissor we have shown operative: failure at $M_{X}$ to fit fermion spectra unless the sfermion and gaugino spectrum is such as to push the down quark type fermion Yukawa couplings above $M_{S}$ to values where they match with the otherwise accurate and consistent fits we find at $M_{X}$ and failure to fit the neutrino masses if the $\overline{\mathbf{1 2 6}}$ couplings are used to correct the second generation mass patterns.

In sum, the NMSGUT having inherited the strengths of its parent is revealing new virtues as well as new weaknesses and, while threatening still to plunge into the yawning crevasse of falsification, yet promises to carry the long winding caravan of Grand Unification not only across the Grand Desert that set its first horizons but across threshold jungles beyond that first horizon up into the rarefied heights where gauge forces and gravity meld into their primordial pleromal [68] unity. 


\section{Acknowledgments}

The work of C.S.A. was supported by grant No. SR/S2/HEP-11/2005 from the Department of Science and Technology of the Government of India and that of S.K.G. by a University Grants Commission Senior Research Fellowship. It is a pleasure for C.S.A. to acknowledge the hospitality of the High Energy Theory Group ICTP,Trieste and in particular Goran Senjanovic. C.S.A. thanks Goran Senjanovic for frank conversations on where MSGUTs stand today as well as Borut Bajc and Alejandra Melfo for discussions on supersymmetry breaking. We thank Sunil Mittal for writing the first version of the 2-loop renormalization group flow Mathematica program that we use as a cross check. C.S.A. acknowledges useful correspondence with S. Antusch and W.A. Porod and informative discussions with K. Babu and Ts. Enkhbat. C.S.A. also thanks Ila Garg and Charanjit Kaur for help with the preparation of the final manuscript and especially the tables.

\section{Note added in proof}

On 13 December 2011, just before we received the proofs, the ATLAS-CMS $5 f b^{-1}$ Higgs mass results indicating $M_{h^{0}} \sim 125 \mathrm{GeV}$ were announced. It is amusing to see that our second example fit coincides with this result. More seriously such Higgs mass values indicate heavy sparticle spectra and large $A_{0}$ values : as many of the plethora of "instantaneous papers" released on December 14 and 15 have noted. Our spectra are strongly of the large $A_{0}$ type but stand out as falsifiable and distinct due to the normal sparticle hierarchy with ultra heavy stops and various other distinctive features like light smuons. They are also distinct in that they necessarily embrace $A_{0}, \mu$ values so large as to render the MSSM vacuum stable against decay to the CCB vacua that large $A_{0}, \mu$ parameters imply [71]. We emphasize that these features of the sparticle spectra are relatively independent of the details by which the GUT achieves fits of the fermion spectra at $M_{X}^{0}$. They are required by the need to lower $y_{d, s}$ while preserving $y_{b}$.

\section{A.}

\section{A.1. MSGUT couplings and vevs.}

A We provide some further details concerning the MSGUT in this appendix for the readers reference. The superpotential contains the mass parameters

$$
m: \mathbf{2 1 0} \quad ; \quad M: \mathbf{1 2 6} \cdot \overline{\mathbf{1 2 6}} ; \quad M_{H}: \mathbf{1 0}^{\mathbf{2}}
$$

and trilinear couplings

$$
\lambda: \mathbf{2 1 0}{ }^{3} \quad ; \quad \eta: \mathbf{2 1 0} \cdot \mathbf{1 2 6} \cdot \overline{\mathbf{1 2 6}} ; \quad \gamma \oplus \bar{\gamma}: \mathbf{1 0} \cdot \mathbf{2 1 0} \cdot(\mathbf{1 2 6} \oplus \overline{\mathbf{1 2 6}})
$$

In addition one has two complex symmetric matrices $h_{A B}, f_{A B}$ of Yukawa couplings of the $\mathbf{1 0}, \overline{\mathbf{1 2 6}}$ Higgs multiplets to the $\mathbf{1 6 . 1 6}$ matter bilinears. The $U(3)$ ambiguity due to $S O(10)$ 'flavor' redefinitions can be used to remove 9 of the 24 real parameters in $f, h$. In addition rephasing of the remaining 4 fields $\mathbf{\Phi}, \mathbf{H}, \boldsymbol{\Sigma}, \overline{\boldsymbol{\Sigma}}$ removes 4 phases from the 14 parameters in $m, M, M_{H}, \lambda, \eta, \gamma, \bar{\gamma}$ leaving 25 superpotential parameters to begin with. Strictly speaking, since a fine tuning to keep one pair of doublets light is an intrinsic part of the MSGUT scenario, an additional complex parameter (say $M_{H}$ ) may be considered as fixed so that there are actually 23 free superpotential 
parameters. In addition the electroweak scale vev $v_{W}$, and $\tan \beta$ are relevant external parameters for the light fermion spectrum determined by the GUT Yukawa structures. The overall superheavy scale(identified with the real parameter $m_{210}$ ) is fixed by the identification of the unification scale determined by the RG flow with the mass of the gauge $X\left[3,2, \pm \frac{4}{3}\right]$ sub-multiplet.

The GUT scale vevs that break the gauge symmetry down to the SM symmetry (see [3] for complete details) are [10, 11]

$$
\begin{array}{rll}
\langle(15,1,1)\rangle_{210} & :\left\langle\phi_{a b c d}\right\rangle=\frac{a}{2} \epsilon_{a b c d e f} \epsilon_{e f} \\
\langle(15,1,3)\rangle_{210} & : \quad\left\langle\phi_{a b \tilde{\alpha} \tilde{\beta}}\right\rangle=\omega \epsilon_{a b} \epsilon_{\tilde{\alpha} \tilde{\beta}} \\
\langle(1,1,1)\rangle_{210} & : \quad\left\langle\phi_{\tilde{\alpha} \tilde{\beta} \tilde{\gamma} \tilde{\delta}}\right\rangle=p \epsilon_{\tilde{\alpha} \tilde{\beta} \tilde{\gamma} \tilde{\delta}} \\
\langle(10,1,3)\rangle_{\overline{126}} & :\left\langle\bar{\Sigma}_{\hat{1} \hat{3} \hat{5} \hat{\delta} \hat{0}}\right\rangle=\bar{\sigma} \\
\langle(\overline{10}, 1,3)\rangle_{126} & : \quad\left\langle\Sigma_{\hat{2} \hat{4} \hat{\bar{c}} \hat{\gamma} \hat{\gamma}}\right\rangle=\sigma .
\end{array}
$$

In the above $a, b . .=1 . .6$ are $\mathrm{SO}(6)$ indices and $\tilde{\alpha}, \tilde{\beta} . .=7 . .10$ are $\mathrm{SO}(4)$ indices and hats indicate their pairwise complexification.

The vanishing of the D-terms of the $\mathrm{SO}(10)$ gauge sector potential imposes only the condition $|\sigma|=|\bar{\sigma}|$. The relevant system of (electrically neutral) superpotential extremization equations $F_{a, p, \omega, \sigma, \bar{\sigma}}=0$ can be reduced to a single cubic equation [12] for a variable $x=-\lambda \omega / m$, in terms of which the vevs $a, \omega, p, \sigma, \bar{\sigma}$ are specified :

$$
C(x, \xi)=8 x^{3}-15 x^{2}+14 x-3+\xi(1-x)^{2}=0
$$

where $\xi=\frac{\lambda M}{\eta m}$. Then the dimensionless vevs in units of $(\mathrm{m} / \lambda)$ are $\tilde{\omega}=-x[12$ and

$$
\tilde{a}=\frac{\left(x^{2}+2 x-1\right)}{(1-x)} \quad ; \quad \tilde{p}=\frac{x\left(5 x^{2}-1\right)}{(1-x)^{2}} \quad ; \quad \tilde{\sigma} \tilde{\bar{\sigma}}=\frac{2}{\eta} \frac{\lambda x(1-3 x)\left(1+x^{2}\right)}{(1-x)^{2}}
$$

This exhibits the crucial importance of the parameters $\xi, x$. Note that one can trade [4,5, 12, the parameter $\xi$ for $x$ with advantage using equation (A.8) since $\xi$ is uniquely fixed by $x$. By a survey of the behavior of the theory as a function of the complex parameter $x$ we thus cover the behavior of the three different solutions possible for each complex value of $\xi$.

Among the mass matrices is the all important Higgs doublet mass matrix [3, 6] $\mathcal{H}$ which can be diagonalized by a bi-unitary transformation [4,6, 12]: from the pairs of Higgs doublets $h^{(i)}, \bar{h}^{(i)}$ arising from the $\mathrm{SO}(10)$ fields to a new set $H^{(i)}, \bar{H}^{(i)}$ of fields in terms of which the doublet mass terms are diagonal.

$$
\begin{aligned}
\bar{U}^{T} \mathcal{H} U & =\operatorname{Diag}\left(m_{H}^{(1)}, m_{H}^{(2)}, \ldots .\right) \\
h^{(i)} & =U_{i j} H^{(j)} \quad ; \quad \bar{h}^{(i)}=\bar{U}_{i j} \bar{H}^{(j)}
\end{aligned}
$$

To keep one pair of these doublets light one tunes $M_{H}$ so that DetH $=0$. In the effective theory at low energies the GUT Higgs doublets $h^{(i)}, \bar{h}^{(i)}$ are present in the massless doublets $H^{(1)}, \bar{H}^{(1)}$ in a proportion determined by the first columns of the matrices $U, \bar{U}$ :

$$
\begin{array}{ccccc}
E<<M_{X} \quad: \quad h^{(i)} & \rightarrow \alpha_{i} H^{(1)} \quad ; \quad \alpha_{i}=U_{i 1} \\
& \bar{h}^{(i)} & \rightarrow \bar{\alpha}_{i} \bar{H}^{(1)} \quad ; \quad \bar{\alpha}_{i}=\bar{U}_{i 1}
\end{array}
$$

The all important normalized 6 -tuples $\alpha, \bar{\alpha}$ can be easily determined [4, 5, 12, 15, 16] by solving the zero mode conditions: $\mathcal{H} \alpha=0 ; \bar{\alpha}^{T} \mathcal{H}=0$. Explicit formulae may be found in [25]. We use a convention where the first component i.e $\alpha_{1}, \bar{\alpha}_{1}$ is real. 


\section{A.2. 120-plet superpotential}

The Pati-Salam decomposition of the rest of the 120-plet terms terms in the superpotential is :

$$
\begin{aligned}
\frac{m_{\Theta}}{2(3 !)} \Theta_{i j k} \Theta_{i j k}= & \frac{m_{\Theta}}{12}\left[6 \Theta_{\mu \nu}^{(s)} \Theta_{(s)}^{\mu \nu}+6 \Theta_{\sigma}^{\lambda \alpha \dot{\alpha}} \Theta_{\lambda \alpha \dot{\alpha}}^{\sigma}\right. \\
& +3\left(\overrightarrow{\widetilde{\Theta}}_{(a)(R)}^{\mu \nu} \cdot \vec{\Theta}_{\mu \nu(R)}^{(a)}+\overrightarrow{\widetilde{\Theta}}_{(a)(L)}^{\mu \nu} \cdot \vec{\Theta}_{\mu \nu(L)}^{(a)}\right) \\
& \left.-6 \Theta^{\alpha \dot{\alpha}} \Theta_{\alpha \dot{\alpha}}\right]
\end{aligned}
$$

$$
\begin{aligned}
& \frac{\rho}{4 !} \Theta_{i j m} \Theta_{k l m} \Phi_{i j k l}=\frac{\rho}{4 !}\left[8 i \Theta_{\mu \lambda}^{(s)} \Theta_{(s)}^{\nu \lambda} \Phi_{\nu}{ }^{\mu}+8 i \Theta_{\sigma}^{\mu \alpha \dot{\alpha}} \Theta_{\nu \alpha \dot{\alpha}}^{\sigma} \Phi_{\mu}{ }^{\nu}\right. \\
& -4 \sqrt{2}\left(\Theta_{\mu \lambda}^{(s)} \overrightarrow{\tilde{\Theta}}_{(a)(R)}^{\mu \nu} \cdot \vec{\Phi}_{\nu(R)}^{\lambda}+\Theta_{\mu \lambda}^{(s)} \overrightarrow{\tilde{\Theta}}_{(a)(L)}^{\mu \nu} \cdot \vec{\Phi}_{\nu(L)}^{\lambda}\right. \\
& \left.-\Theta_{(s)}^{\mu \lambda} \vec{\Theta}_{\mu \nu(R)}^{(a)} \cdot \vec{\Phi}_{\lambda(R)}^{\nu}-\Theta_{(s)}^{\mu \lambda} \vec{\Theta}_{\mu \nu(L)}^{(a)} \cdot \vec{\Phi}_{\lambda(L)}^{\nu}\right) \\
& +4 \sqrt{2}\left(\Theta^{\alpha \dot{\alpha}} \Theta_{\nu \alpha}^{\mu \dot{\beta}} \Phi_{\mu \dot{\alpha} \dot{\beta}}^{\nu}-\Theta^{\alpha \dot{\alpha}} \Theta_{\nu \dot{\alpha}}^{\mu \beta} \Phi_{\mu \alpha \beta}^{\nu}\right) \\
& +8\left(\Theta_{\nu}{ }^{\mu \alpha \dot{\alpha}} \Theta_{\mu \lambda}^{(s)} \Phi_{\alpha \dot{\alpha}}^{\nu \lambda(s)}-\Theta_{\nu}{ }^{\mu \alpha \dot{\alpha}} \Theta_{(s)}^{\nu \lambda} \Phi_{\mu \lambda \alpha \dot{\alpha}}^{(s)}\right) \\
& -2\left(\tilde{\Theta}_{(a) \dot{\alpha} \dot{\beta}}^{\mu \nu} \Theta_{\nu \alpha}^{\lambda \dot{\beta}} \Phi_{\mu \lambda(s)}^{\alpha \dot{\alpha}}+\tilde{\Theta}_{(a) \alpha \beta}^{\mu \nu} \Theta_{\nu \dot{\alpha}}^{\lambda \beta} \Phi_{\mu \lambda(s)}^{\alpha \dot{\alpha}}\right. \\
& \left.-\Theta_{\mu \nu \dot{\alpha} \dot{\beta}}^{(a)} \Theta_{\lambda \alpha}^{\nu \dot{\beta}} \Phi_{(s)}^{\mu \lambda \alpha \dot{\alpha}}-\Theta_{\mu \nu \alpha \beta}^{(a)} \Theta_{\lambda \dot{\alpha}}^{\nu \beta} \Phi_{(s)}^{\mu \lambda \alpha \dot{\alpha}}\right) \\
& -2\left(\overrightarrow{\tilde{\Theta}}_{(a)(L)}^{\mu \nu} \cdot \vec{\Theta}_{\mu \nu(L)}^{(a)}-\overrightarrow{\tilde{\Theta}}_{(a)(R)}^{\mu \nu} \cdot \vec{\Theta}_{\mu \nu(R)}^{(a)}\right) \Phi \\
& -4 \sqrt{2}\left(\Theta_{\lambda}^{\mu \alpha \dot{\alpha}} \Theta_{(a) \dot{\alpha} \dot{\beta}}^{\nu \lambda} \Phi_{\mu \nu \alpha}^{(a) \dot{\beta}}-\Theta_{\lambda}^{\mu \alpha \dot{\alpha}} \Theta_{(a) \alpha \beta}^{\nu \lambda} \Phi_{\mu \nu \dot{\alpha}}^{(a) \beta}\right) \\
& -2 \sqrt{2}\left(\Theta^{\alpha \dot{\alpha}} \tilde{\Theta}_{(a) \dot{\alpha} \dot{\beta}}^{\mu \nu} \Phi_{\mu \nu \alpha}^{(a) \dot{\beta}}+\Theta^{\alpha \dot{\alpha}} \tilde{\Theta}_{(a) \alpha \beta}^{\mu \nu} \Phi_{\mu \nu \dot{\alpha}}^{(a) \beta}\right) \\
& +4 \sqrt{2}\left(\Theta_{\lambda}^{\nu \alpha \dot{\alpha}} \Theta_{\mu \alpha}^{\lambda \dot{\beta}} \Phi_{\nu \dot{\alpha} \dot{\beta}}^{\mu}+\Theta_{\lambda}^{\nu \alpha \dot{\alpha}} \Theta_{\mu \dot{\alpha}}^{\lambda \beta} \Phi_{\nu \alpha \beta}^{\mu}\right) \\
& -2\left(\Theta_{\nu \alpha}^{\lambda \dot{\beta}} \tilde{\Theta}_{\dot{\alpha} \dot{\beta}}^{\mu \nu(a)} \Phi_{\mu \lambda(s)}^{\alpha \dot{\alpha}}+\Theta_{\nu \dot{\alpha}}^{\lambda \beta} \tilde{\Theta}_{\alpha \beta}^{\mu \nu(a)} \Phi_{\mu \lambda(s)}^{\alpha \dot{\alpha}}\right. \\
& \text { - } \left.\Theta_{\lambda \dot{\alpha}}^{\nu \beta} \Theta_{\mu \nu \alpha \beta}^{(a)} \Phi_{(s)}^{\mu \lambda \alpha \dot{\alpha}}-\Theta_{\lambda \alpha}^{\nu \dot{\beta}} \Theta_{\mu \nu \dot{\alpha} \dot{\beta}}^{(a)} \Phi_{(s)}^{\mu \lambda \alpha \dot{\alpha}}\right) \\
& -4 \sqrt{2}\left(\vec{\Theta}_{\mu \nu(R)}^{(a)} \cdot\left(\vec{\Theta}_{(a) R}^{\nu \lambda} \times \vec{\Phi}_{\lambda(R)}^{\mu}\right)\right. \\
& \left.\left.+\vec{\Theta}_{\mu \nu(L)}^{(a)} \cdot\left(\vec{\Theta}_{(a) L}^{\nu \lambda} \times \vec{\Phi}_{\lambda(L)}^{\mu}\right)\right)\right]
\end{aligned}
$$




$$
\begin{aligned}
& \frac{\zeta}{2(3 !)} \Theta_{i j k} \Sigma_{i j l m n} \Phi_{k l m n}=\frac{\zeta}{2(3 !)}\left[-6 \sqrt{2} i\left(\Theta_{\mu \lambda}^{(s)} \tilde{\Sigma}_{(a)}^{\mu \nu} \Phi_{\nu}{ }^{\lambda}-\Theta_{(s)}^{\mu \lambda} \Sigma_{\mu \nu}^{(a)} \Phi_{\lambda}{ }^{\nu}\right)\right. \\
& -12 i\left(\Theta_{\mu \lambda}^{(s)} \Sigma_{\nu}^{\mu \alpha \dot{\alpha}} \Phi_{\alpha \dot{\alpha}}^{\nu \lambda(s)}+\Theta_{(s)}^{\nu \lambda} \Sigma_{\nu}^{\mu \alpha \dot{\alpha}} \Phi_{\mu \lambda \alpha \dot{\alpha}}^{(s)}\right) \\
& -12 \sqrt{2}\left(\Theta_{\mu \lambda}^{(s)} \vec{\Sigma}_{(s)(R)}^{\nu \lambda} \cdot \vec{\Phi}_{\nu(R)}^{\mu}-\Theta_{(s)}^{\nu \lambda} \vec{\Sigma}_{\mu \lambda(L)}^{(s)} \cdot \vec{\Phi}_{\nu(L)}^{\mu}\right) \\
& +6 \sqrt{2} i\left(\Theta_{(s)}^{\mu \lambda} \Sigma_{\lambda \alpha \dot{\alpha}}^{\nu} \Phi_{\mu \nu(a)}^{\alpha \dot{\alpha}}-\Theta_{\mu \lambda}^{(s)} \Sigma_{\nu \alpha \dot{\alpha}}^{\lambda} \tilde{\Phi}_{(a)}^{\mu \nu \alpha \dot{\alpha}}\right) \\
& -6 \sqrt{2}\left(\Theta_{\nu}^{\lambda \alpha \dot{\alpha}} \tilde{\Sigma}_{(a)}^{\mu \nu} \Phi_{\mu \lambda \alpha \dot{\alpha}}^{(s)}+\Theta_{\lambda}^{\nu \alpha \dot{\alpha}} \Sigma_{\mu \nu}^{(a)} \Phi_{\alpha \dot{\alpha}}^{\mu \lambda(s)}\right) \\
& -12 \sqrt{2} i\left(\Theta_{\lambda}^{\mu \alpha \dot{\alpha}} \Sigma_{\nu \dot{\alpha}}^{\lambda \beta} \Phi_{\mu \alpha \beta}^{\nu}+\Theta_{\lambda}^{\mu \alpha \dot{\alpha}} \Sigma_{\mu \alpha}^{\nu \dot{\beta}} \Phi_{\nu \dot{\alpha} \dot{\beta}}^{\lambda}\right) \\
& +6 \sqrt{2}\left(\Theta_{\nu \dot{\alpha}}^{\lambda \beta} \Sigma_{\mu \lambda \alpha \beta}^{(s)} \tilde{\Phi}_{(a)}^{\mu \nu \alpha \dot{\alpha}}+\Theta_{\lambda \alpha}^{\nu \dot{\beta}} \Sigma_{\dot{\alpha} \dot{\beta}}^{\mu \lambda(s)} \Phi_{\mu \nu(a)}^{\alpha \dot{\alpha}}\right) \\
& +12 i \Theta_{\nu}^{\mu \alpha \dot{\alpha}} \Sigma_{\mu \alpha \dot{\alpha}}^{\nu} \Phi \\
& +12 i\left(\overrightarrow{\tilde{\Theta}}_{(a)(L)}^{\mu \nu} \cdot \vec{\Sigma}_{\mu \lambda(L)}^{(s)} \Phi_{\nu}{ }^{\lambda}+\vec{\Theta}_{\mu \nu(R)}^{(a)} \cdot \vec{\Sigma}_{(s)(R)}^{\mu \lambda} \Phi_{\lambda}^{\nu}\right) \\
& -6 i\left(\tilde{\Theta}_{(a) \dot{\alpha} \dot{\beta}}^{\mu \nu} \Sigma_{\nu \alpha}^{\lambda \dot{\beta}} \Phi_{\mu \lambda(s)}^{\alpha \dot{\alpha}}-\tilde{\Theta}_{(a) \alpha \beta}^{\mu \nu} \Sigma_{\nu \dot{\alpha}}^{\lambda \beta} \Phi_{\mu \lambda(s)}^{\alpha \dot{\alpha}}\right. \\
& \left.-\Theta_{\mu \nu \dot{\alpha} \dot{\beta}}^{(a)} \Sigma_{\lambda \alpha}^{\nu \dot{\beta}} \Phi_{(s)}^{\mu \lambda \alpha \dot{\alpha}}+\Theta_{\mu \nu \alpha \beta}^{(a)} \Sigma_{\lambda \dot{\alpha}}^{\nu \beta} \Phi_{(s)}^{\mu \lambda \alpha \dot{\alpha}}\right) \\
& -12\left(\vec{\Theta}_{\mu \nu(L)}^{(a)} \vec{\Phi}_{\lambda(L)}^{\mu}-\vec{\Theta}_{\mu \nu(R)}^{(a)} \vec{\Phi}_{\lambda(R)}^{\mu}\right) \Sigma_{(a)}^{\nu \lambda} \\
& +6\left(\Theta_{\dot{\alpha}}^{\beta} \Sigma_{\mu \nu \alpha \beta}^{(s)} \Phi_{(s)}^{\mu \nu \alpha \dot{\alpha}}-\Theta_{\alpha}^{\dot{\beta}} \Sigma_{\dot{\alpha} \dot{\beta}}^{\mu \nu(s)} \Phi_{\mu \nu(s)}^{\alpha \dot{\alpha}}\right) \\
& -6 \sqrt{2} i\left(\Theta_{\alpha}^{\dot{\beta}} \Sigma_{\nu}^{\mu \alpha \dot{\alpha}} \Phi_{\mu \dot{\alpha} \dot{\beta}}^{\nu}+\Theta_{\dot{\alpha}}^{\beta} \Sigma_{\nu}{ }^{\mu \alpha \dot{\alpha}} \Phi_{\mu \alpha \beta}^{\nu}\right) \\
& +12\left(\Theta_{\nu}^{\mu \alpha \dot{\alpha}} \Sigma_{\lambda \alpha \dot{\alpha}}^{\nu} \Phi_{\mu}^{\lambda}-\Theta_{\nu}^{\mu \alpha \dot{\alpha}} \Sigma_{\mu \alpha \dot{\alpha}}^{\lambda} \Phi_{\lambda}^{\nu}\right) \\
& +12\left(\Theta_{\nu}{ }^{\mu \alpha \dot{\alpha}} \Sigma_{\mu \lambda \alpha \beta}^{(s)} \Phi_{(s) \dot{\alpha}}^{\nu \lambda \beta}-\Theta_{\nu}^{\mu \alpha \dot{\alpha}} \Sigma_{(s) \dot{\alpha} \dot{\beta}}^{\nu \lambda} \Phi_{\mu \lambda \alpha}^{(s) \dot{\beta}}\right) \\
& -12\left(\Theta_{\lambda}^{\mu \alpha \dot{\alpha}} \Sigma_{\mu \nu}^{(a)} \Phi_{(a) \alpha \dot{\alpha}}^{\nu \lambda}\right) \\
& +6 i\left(\tilde{\Theta}_{(a) \dot{\alpha} \dot{\beta}}^{\mu \nu} \Sigma_{\nu}^{\lambda \alpha \dot{\alpha}} \Phi_{\mu \lambda \alpha}^{(s) \dot{\beta}}+\tilde{\Theta}_{(a) \alpha \beta}^{\mu \nu} \Sigma_{\nu}^{\lambda \alpha \dot{\alpha}} \Phi_{\mu \lambda \dot{\alpha}}^{(s) \beta}\right. \\
& \left.+\Theta_{\mu \nu \dot{\alpha} \dot{\beta}}^{(a)} \Sigma_{\lambda}^{\nu \alpha \dot{\alpha}} \Phi_{(s) \alpha}^{\mu \lambda \dot{\beta}}+\Theta_{\mu \nu \alpha \beta}^{(a)} \Sigma_{\lambda}^{\nu \alpha \dot{\alpha}} \Phi_{(s) \dot{\alpha}}^{\mu \lambda \beta}\right) \\
& +12 \sqrt{2}\left[\overrightarrow{\tilde{\Theta}}_{(a)(L)}^{\mu \nu} \cdot\left(\vec{\Phi}_{\nu(L)}^{\lambda} \times \vec{\Sigma}_{\mu \lambda(L)}^{(s)}\right)\right. \\
& \left.-\vec{\Theta}_{\mu \nu(R)}^{(a)} \cdot\left(\vec{\Phi}_{\lambda(R)}^{\nu} \times \vec{\Sigma}_{(R)}^{\mu \lambda(s)}\right)\right] \\
& \left.+6 \sqrt{2} i\left(\Theta_{\mu \nu \dot{\alpha} \dot{\beta}}^{(a)} \Sigma_{\lambda \alpha}^{\mu \dot{\beta}} \Phi_{(a)}^{\nu \lambda \alpha \dot{\alpha}}+\Theta_{\mu \nu \alpha \beta}^{(a)} \Sigma_{\lambda \dot{\alpha}}^{\mu \beta} \Phi_{(a)}^{\nu \lambda \alpha \dot{\alpha}}\right)\right]
\end{aligned}
$$




$$
\begin{aligned}
& \frac{\bar{\zeta}}{2(3 !)} \Theta_{i j k} \bar{\Sigma}_{i j l m n} \Phi_{k l m n}=\frac{\bar{\zeta}}{2(3 !)}\left[6 \sqrt{2} i\left(\Theta_{\mu \lambda}^{(s)} \tilde{\bar{\Sigma}}_{(a)}^{\mu \nu} \Phi_{\nu}{ }^{\lambda}-\Theta_{(s)}^{\mu \lambda} \bar{\Sigma}_{\mu \nu}^{(a)} \Phi_{\lambda}{ }^{\nu}\right)\right. \\
& -12 i\left(\Theta_{\mu \lambda}^{(s)} \bar{\Sigma}_{\nu}^{\mu \alpha \dot{\alpha}} \Phi_{\alpha \dot{\alpha}}^{\nu \lambda(s)}+\Theta_{(s)}^{\nu \lambda} \bar{\Sigma}_{\nu}^{\mu \alpha \dot{\alpha}} \Phi_{\mu \lambda \alpha \dot{\alpha}}^{(s)}\right) \\
& -12 \sqrt{2}\left(\Theta_{\mu \lambda}^{(s)} \vec{\Sigma}_{(L)}^{\nu \lambda(s)} \cdot \vec{\Phi}_{\nu(L)}^{\mu}-\Theta_{(s)}^{\nu \lambda} \vec{\Sigma}_{\mu \lambda(R)}^{(s)} \cdot \vec{\Phi}_{\nu(R)}^{\mu}\right) \\
& -6 \sqrt{2} i\left(\Theta_{(s)}^{\mu \lambda} \bar{\Sigma}_{\lambda \alpha \dot{\alpha}}^{\nu} \Phi_{\mu \nu}^{(a) \alpha \dot{\alpha}}-\Theta_{\mu \lambda}^{(s)} \bar{\Sigma}_{\nu \alpha \dot{\alpha}}^{\lambda} \tilde{\Phi}_{(a)}^{\mu \nu \alpha \dot{\alpha}}\right) \\
& +6 \sqrt{2}\left(\Theta_{\nu}^{\lambda \alpha \dot{\alpha}} \tilde{\bar{\Sigma}}_{(a)}^{\mu \nu} \Phi_{\mu \lambda \alpha \dot{\alpha}}^{(s)}+\Theta_{\lambda}^{\nu \alpha \dot{\alpha}} \bar{\Sigma}_{\mu \nu}^{(a)} \Phi_{\alpha \dot{\alpha}}^{\mu \lambda(s)}\right) \\
& -12 \sqrt{2} i\left(\Theta_{\lambda}^{\mu \alpha \dot{\alpha}} \bar{\Sigma}_{\nu \alpha}^{\lambda \dot{\beta}} \Phi_{\mu \dot{\alpha} \dot{\beta}}^{\nu}+\Theta_{\lambda}^{\mu \alpha \dot{\alpha}} \bar{\Sigma}_{\mu \dot{\alpha}}^{\nu \beta} \Phi_{\nu \alpha \beta}^{\lambda}\right) \\
& -6 \sqrt{2}\left(\Theta_{\nu \alpha}^{\lambda \dot{\beta}} \bar{\Sigma}_{\mu \lambda \dot{\alpha} \dot{\beta}}^{(s)} \tilde{\Phi}_{(a)}^{\mu \nu \alpha \dot{\alpha}}+\Theta_{\lambda \dot{\alpha}}^{\nu \beta} \bar{\Sigma}_{\alpha \beta}^{\mu \lambda(s)} \Phi_{\mu \nu}^{(a) \alpha \dot{\alpha}}\right) \\
& -12 i \Theta_{\nu}^{\mu \alpha \dot{\alpha}} \bar{\Sigma}_{\mu \alpha \dot{\alpha}}^{\nu} \Phi \\
& +12 i\left(\overrightarrow{\tilde{\Theta}}_{(R)}^{\mu \nu(a)} \cdot \vec{\Sigma}_{\mu \lambda(R)}^{(s)} \Phi_{\nu}^{\lambda}+\vec{\Theta}_{\mu \nu(L)}^{(a)} \cdot \vec{\Sigma}_{(L)}^{\mu \lambda(s)} \Phi_{\lambda}^{\nu}\right) \\
& +6 i\left(\tilde{\Theta}_{\dot{\alpha} \dot{\beta}}^{\mu \nu(a)} \bar{\Sigma}_{\nu \alpha}^{\lambda \dot{\beta}} \Phi_{\mu \lambda(a)}^{\alpha \dot{\alpha}}-\tilde{\Theta}_{\alpha \beta}^{\mu \nu(a)} \bar{\Sigma}_{\nu \dot{\alpha}}^{\lambda \beta} \Phi_{\mu \lambda(a)}^{\alpha \dot{\alpha}}\right. \\
& \left.-\Theta_{\mu \nu \dot{\alpha} \dot{\beta}}^{(a)} \bar{\Sigma}_{\lambda \alpha}^{\nu \dot{\beta}} \Phi_{(a)}^{\mu \lambda \alpha \dot{\alpha}}+\Theta_{\mu \nu \alpha \beta}^{(a)} \bar{\Sigma}_{\lambda \dot{\alpha}}^{\nu \beta} \Phi_{(a)}^{\mu \lambda \alpha \dot{\alpha}}\right) \\
& -12\left(\vec{\Theta}_{\mu \nu(L)}^{(a)} \cdot \vec{\Phi}_{\lambda(L)}^{\mu}-\vec{\Theta}_{\mu \nu(R)}^{(a)} \cdot \vec{\Phi}_{\lambda(R)}^{\mu}\right) \bar{\Sigma}_{(a)}^{\nu \lambda} \\
& -6\left(\Theta_{\alpha}^{\dot{\beta}} \bar{\Sigma}_{\mu \nu \dot{\alpha} \dot{\beta}}^{(s)} \Phi_{(s)}^{\mu \nu \alpha \dot{\alpha}}-\Theta_{\dot{\alpha}}^{\beta} \bar{\Sigma}_{\alpha \beta}^{\mu \nu(s)} \Phi_{\mu \nu(s)}^{\alpha \dot{\alpha}}\right) \\
& -6 \sqrt{2} i\left(\Theta_{\alpha}^{\dot{\beta}} \bar{\Sigma}_{\nu}^{\mu \alpha \dot{\alpha}} \Phi_{\mu \dot{\alpha} \dot{\beta}}^{\nu}+\Theta_{\dot{\alpha}}^{\beta} \bar{\Sigma}_{\nu}^{\mu \alpha \dot{\alpha}} \Phi_{\mu \alpha \beta}^{\nu}\right) \\
& +12\left(\Theta_{\nu}^{\mu \alpha \alpha \dot{\alpha}} \bar{\Sigma}_{\lambda \alpha \dot{\alpha}}^{\nu} \Phi_{\mu}^{\lambda}-\Theta_{\nu}{ }^{\mu \alpha \dot{\alpha}} \bar{\Sigma}_{\mu \alpha \dot{\alpha}}^{\lambda} \Phi_{\lambda}^{\nu}\right) \\
& +12\left(\Theta_{\nu}^{\mu \alpha \dot{\alpha}} \bar{\Sigma}_{\mu \lambda \dot{\alpha} \dot{\beta}}^{(s)} \Phi_{\alpha}^{\nu \lambda(s) \dot{\beta}}-\Theta_{\nu}^{\mu \alpha \dot{\alpha}} \bar{\Sigma}_{\alpha \beta}^{\nu \lambda(s)} \Phi_{\mu \lambda(s) \dot{\alpha}}^{\beta}\right) \\
& -12\left(\Theta_{\lambda}^{\mu \alpha \dot{\alpha}} \bar{\Sigma}_{\mu \nu}^{(a)} \Phi_{(a) \alpha \dot{\alpha}}^{\nu \lambda}\right) \\
& +6 i\left(\tilde{\Theta}_{(a) \dot{\alpha} \dot{\beta}}^{\mu \nu} \bar{\Sigma}_{\nu}^{\lambda \alpha \dot{\alpha}} \Phi_{\mu \lambda \alpha}^{(s) \dot{\beta}}+\tilde{\Theta}_{(a) \alpha \beta}^{\mu \nu} \bar{\Sigma}_{\nu}^{\lambda \alpha \dot{\alpha}} \Phi_{\mu \lambda \dot{\alpha}}^{(s) \beta}\right. \\
& \left.+\Theta_{\mu \nu \dot{\alpha} \dot{\beta}}^{(a)} \bar{\Sigma}_{\lambda}^{\nu \alpha \dot{\alpha}} \Phi_{\alpha}^{\mu \lambda(s) \dot{\beta}}+\Theta_{\mu \nu \alpha \beta}^{(a)} \bar{\Sigma}_{\lambda}^{\nu \alpha \dot{\alpha}} \Phi_{\dot{\alpha}}^{\mu \lambda(s) \beta}\right) \\
& +12 \sqrt{2}\left[\overrightarrow{\tilde{\Theta}}_{(a)(R)}^{\mu \nu} \cdot\left(\vec{\Phi}_{\nu(R)}^{\lambda} \times \vec{\Sigma}_{\mu \lambda(R)}^{(s)}\right)\right. \\
& \left.-\vec{\Theta}_{\mu \nu(L)}^{(a)} \cdot\left(\vec{\Phi}_{\lambda(L)}^{\nu} \times \vec{\Sigma}_{(s)(L)}^{\mu \lambda}\right)\right] \\
& \left.-6 \sqrt{2} i\left(\Phi_{(a)}^{\nu \lambda \alpha \dot{\alpha}} \bar{\Sigma}_{\lambda \alpha}^{\mu \dot{\beta}} \Theta_{\mu \nu \dot{\alpha} \dot{\beta}}^{(a)}+\Phi_{(a)}^{\nu \lambda \alpha \dot{\alpha}} \bar{\Sigma}_{\lambda \dot{\alpha}}^{\mu \beta} \Theta_{\mu \nu \alpha \beta}^{(a)}\right)\right]
\end{aligned}
$$

\section{B. : Tables of NMSGUT mass matrices}

Here superfield mass matrix rows are labeled by barred irreps and columns by unbarred.

(i) The 26 letters of the English alphabet are just sufficient to label the 26 distinct MSSM multiplet types that occur in the (N)MSGUT. Calligraphic letters denote the superfield mass matrices of the corresponding alphabetically named MSSM multiplet type. The masses of 13 Unmixed fields are given in Table 2 . 


\begin{tabular}{|c|c|c|}
\hline Field $[S U(3), S U(2), Y]$ & Fields & Mass \\
\hline$A[1,1,4], \bar{A}[1,1,-4]$ & $\frac{\Sigma_{(R+)}^{44}}{\sqrt{2}}, \frac{\bar{\Sigma}_{44(R-)}}{\sqrt{2}}$ & $2(M+\eta(p+3 a+6 \omega))$ \\
\hline$M\left[6,1, \frac{8}{3}\right], \bar{M}\left[\left(\overline{6}, 1,-\frac{8}{3}\right]\right.$ & 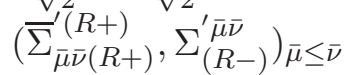 & $2(M+\eta(p-a+2 \omega))$ \\
\hline$N\left[6,1,-\frac{4}{3}\right], \bar{N}\left[\left(\overline{6}, 1, \frac{4}{3}\right]\right.$ & $\left(\bar{\Sigma}_{\bar{\mu} \bar{\nu}}^{\prime}(R-), \Sigma_{(R+)}^{\prime}\right)_{\bar{\mu} \leq \bar{\nu}}$ & $2(M+\eta(p-a-2 \omega))$ \\
\hline$O[1,3,-2], \bar{O}[(1,3,+2]$ & $\frac{\vec{\Sigma}_{44(L)}}{\sqrt{2}}, \frac{\vec{\Sigma}_{(L)}^{44}}{\sqrt{2}}$ & $2(M+\eta(3 a-p))$ \\
\hline$W\left[6,3, \frac{2}{3}\right], \bar{W}\left[\left(\overline{6}, 3,-\frac{2}{3}\right]\right.$ & $\vec{\Sigma}_{\bar{\mu} \bar{\nu}(L)}^{\prime}, \overrightarrow{\bar{\Sigma}}_{(L)}^{\bar{\mu} \bar{\nu}}$ & $2(M-\eta(a+p))$ \\
\hline$I\left[3,1, \frac{10}{3}\right], \bar{I}\left[\left(\overline{3}, 1,-\frac{10}{3}\right]\right.$ & $\phi_{\bar{\nu}(R+)}^{4}, \phi_{4(R-)}^{\bar{\nu}}$ & $-2(m+\lambda(p+a+4 \omega))$ \\
\hline$S[1,3,0]$ & $\vec{\phi}_{(L)}^{(15)}$ & $2(m+\lambda(2 a-p))$ \\
\hline$Q[8,3,0]$ & $\overrightarrow{\phi_{\bar{\mu}}} \bar{\nu}$ & $2(m-\lambda(a+p))$ \\
\hline$U\left[3,3, \frac{4}{3}\right], \bar{U}\left[\overline{3}, 3,-\frac{4}{3}\right]$ & $\vec{\phi}_{\bar{\mu}(L)}^{4}, \vec{\phi}_{4(L)}^{\bar{\mu}}$ & $-2(m-\lambda(p-a))$ \\
\hline$V[1,2,-3], \bar{V}[1,2,3]$ & $\frac{\phi_{44 \alpha \dot{2}}}{\sqrt{2}}, \frac{\phi_{\alpha i}^{44}}{\sqrt{2}}$ & $2(m+3 \lambda(a+\omega))$ \\
\hline$B\left[6,2, \frac{5}{3}\right], \bar{B}\left[\left(\overline{6}, 2,-\frac{5}{3}\right]\right.$ & $\left(\phi_{\bar{\mu} \bar{\nu} \alpha \dot{1}}^{\prime}, \phi_{\alpha \dot{2}}^{\prime}\right)_{\bar{\mu} \leq \bar{\nu}}$ & $-2(m+\lambda(\omega-a))$ \\
\hline$Y\left[6,2,-\frac{1}{3}\right], \bar{Y}\left[\left(\overline{6}, 2, \frac{1}{3}\right]\right.$ & $\left(\phi_{\bar{\mu} \bar{\nu} \alpha \dot{2}}^{\prime}, \phi_{\alpha \dot{1}}^{\prime} \bar{\mu} \bar{\nu}\right)_{\bar{\mu} \leq \bar{\nu}}$ & $2(m-\lambda(a+\omega))$ \\
\hline$Z[8,1,2], \bar{Z}[8,1,-2]$ & $\phi_{\bar{\mu}(R+)}^{\bar{\nu}} \phi_{\bar{\mu}(R-)}^{\bar{\nu}}$ & $2(m+\lambda(p-a))$ \\
\hline
\end{tabular}

Table 2: Masses of the unmixed states in terms of the superheavy vevs . The $S U(2)_{L}$ contraction order is always $\bar{F}^{\alpha} F_{\alpha}$. The absolute value of the expressions in the column "Mass" is understood. For sextets of $S U(3)$ the 6 unit norm fields are denoted by a prime : $\Sigma_{\bar{\mu} \bar{\nu}}^{\prime}=\Sigma_{\bar{\mu} \bar{\nu}}, \bar{\mu}>\bar{\nu}, \Sigma_{\bar{\mu} \bar{\mu}}^{\prime}=\Sigma_{\bar{\mu} \bar{\mu}} / \sqrt{2}$ and similarly for $\overline{6}$. 
ii)

a) $[8,2,-1]\left(\bar{C}_{1}, \bar{C}_{2}, \bar{C}_{3}\right) \bigoplus[8,2,1]\left(C_{1}, C_{2}, C_{3}\right) \equiv\left(\bar{\Sigma}_{\dot{2}}^{A \alpha}, \Sigma_{\dot{2}}^{A \alpha}, \Theta_{\dot{2}}^{A \alpha}\right) \bigoplus$ $\left(\Sigma_{\alpha \mathrm{i}}^{A}, \bar{\Sigma}_{\alpha \mathrm{i}}^{A}, \Theta_{\alpha \mathrm{i}}^{A}\right)(A=1 \ldots . .8)$

$$
\mathcal{C}=\left(\begin{array}{ccc}
2(-M+\eta(a+\omega)) & 0 & -i(\omega-p) \bar{\zeta} \\
0 & 2(-M+\eta(a+\omega)) & -i(\omega+p) \bar{\zeta} \\
i(\omega-p) \zeta & i(\omega+p) \bar{\zeta} & -m_{\Theta}+\frac{\rho}{3} a
\end{array}\right)
$$

b) $\left[\overline{3}, 2,-\frac{7}{3}\right]\left(\bar{D}_{1}, \bar{D}_{2}, \bar{D}_{3}\right) \oplus\left[3,2, \frac{7}{3}\right]\left(D_{1}, D_{2}, D_{3}\right) \equiv\left(\Sigma_{4 \dot{2}}^{\bar{\nu} \alpha}, \bar{\Sigma} \bar{\nu} \alpha, \Theta_{4 \dot{2}}^{\bar{\nu} \alpha}\right) \oplus$ $\left(\bar{\Sigma}_{\bar{\nu} \alpha \mathrm{i}}^{4}, \Sigma_{\bar{\nu} \alpha \mathrm{i}}^{4}, \Theta_{\bar{\nu} \alpha \mathrm{i}}^{4}\right)$

$$
\mathcal{D}=\left(\begin{array}{ccc}
2(M+\eta(a+\omega)) & 0 & (i \omega+i p-2 i a) \zeta \\
0 & 2(M+\eta(a+3 \omega)) & (-3 i \omega-i p-2 i a) \bar{\zeta} \\
-(i \omega+i p-2 i a) \bar{\zeta} & (3 i \omega+i p+2 i a) \zeta & m_{\Theta}+\frac{\rho}{3}(a+2 \omega)
\end{array}\right)
$$

c) $\left[\overline{3}, 2,-\frac{1}{3}\right]\left(\bar{E}_{1}, \bar{E}_{2}, \bar{E}_{3}, \bar{E}_{4}, \bar{E}_{5}, \bar{E}_{6}\right) \oplus\left[3,2, \frac{1}{3}\right]\left(E_{1}, E_{2}, E_{3}, E_{4}, E_{5}, E_{6}\right)$

$$
\begin{aligned}
& \equiv\left(\Sigma_{4 \dot{1}}^{\bar{\mu} \alpha}, \bar{\Sigma}_{4 \dot{1}}^{\bar{\mu} \alpha}, \phi_{(s) \dot{2}}^{\bar{\mu} 4 \alpha}, \phi_{\dot{2}}^{(a) \bar{\mu} 4 \alpha}, \lambda_{\dot{2}}^{\bar{\mu} 4 \alpha}, \Theta_{4 \dot{1}}^{\bar{\sigma} \alpha}\right) \oplus\left(\bar{\Sigma}_{\bar{\mu} \alpha \dot{2}}^{4} \Sigma_{\bar{\mu} \alpha \dot{2}}^{4}, \phi_{\bar{\mu} 4 \alpha \dot{1}}^{(s)}, \phi_{\bar{\mu} 4 \alpha \dot{1}}^{(a)}, \lambda_{\bar{\mu} \alpha \dot{1}}, \Theta_{\bar{\sigma} \alpha}^{4 \dot{1}}\right) \\
& \mathcal{E}=\left(\begin{array}{cccccc}
-2(M+\eta(a-\omega)) & 0 & 0 & 0 & 0 & (i \omega-i p+2 i a) \zeta \\
0 & -2(M+\eta(a-3 \omega)) & -2 \sqrt{2} i \eta \sigma & 2 i \eta \sigma & i g \sqrt{2} \bar{\sigma}^{*} & (-3 i \omega+i p+2 i a) \bar{\zeta} \\
0 & 2 i \sqrt{2} \eta \bar{\sigma} & -2(m+\lambda(a-\omega)) & -2 \sqrt{2} \lambda \omega & 2 g\left(a^{*}-\omega^{*}\right) & -\sqrt{2} \bar{\zeta} \bar{\sigma} \\
0 & -2 i \eta \bar{\sigma} & -2 \sqrt{2} \lambda \omega & -2(m-\lambda \omega) & \sqrt{2} g\left(\omega^{*}-p^{*}\right) & \bar{\sigma} \bar{\zeta} \\
0 & -i g \sqrt{2} \sigma^{*} & 2 g\left(a^{*}-\omega^{*}\right) & g \sqrt{2}\left(\omega^{*}-p^{*}\right) & 0 & 0 \\
(-i \omega+i p-2 i a) \bar{\zeta} & (3 i \omega-i p-2 i a) \zeta & -\sqrt{2} \zeta \sigma & \sigma \zeta & 0 & -\left(m_{\Theta}+\frac{\rho}{3} a-\frac{2}{3} \rho \omega\right)
\end{array}\right)
\end{aligned}
$$

d) $[1,1,-2]\left(\bar{F}_{1}, \bar{F}_{2}, \bar{F}_{3}, \bar{F}_{4}\right) \oplus[1,1,2]\left(F_{1}, F_{2}, F_{3}, F_{4}\right) \equiv\left(\frac{\bar{\Sigma}_{44(R 0)}}{\sqrt{2}}, \Phi_{(R-)}^{(15)}, \lambda_{(R-)}, \frac{\Theta_{44}}{\sqrt{2}}\right) \oplus$ $\left(\frac{\Sigma_{(R 0)}^{44}}{\sqrt{2}}, \Phi_{(R+)}^{(15)}, \lambda_{(R+)}, \frac{\Theta^{44}}{\sqrt{2}}\right)$

$$
\mathcal{F}=\left(\begin{array}{cccc}
2(M+\eta(p+3 a)) & -2 i \sqrt{3} \eta \sigma & -g \sqrt{2} \bar{\sigma}^{*} & -6 i \bar{\zeta} \omega \\
2 i \sqrt{3} \eta \bar{\sigma} & 2(m+\lambda(p+2 a)) & \sqrt{24} i g \omega^{*} & \sqrt{3} \bar{\zeta} \bar{\sigma} \\
-g \sqrt{2} \sigma^{*} & -\sqrt{24} i g \omega^{*} & 0 & 0 \\
6 i \zeta \omega & \sqrt{3} \zeta \sigma & 0 & m_{\Theta}+a \rho
\end{array}\right)
$$

e) $[1,1,0]\left(G_{1}, G_{2}, G_{3}, G_{4}, G_{5}, G_{6}\right) \equiv\left(\phi, \phi^{(15)}, \phi_{(R 0)}^{(15)}, \frac{\Sigma_{(R-)}^{44}}{\sqrt{2}}, \frac{\bar{\Sigma}_{44((R+)}}{\sqrt{2}}, \frac{\sqrt{2} \lambda^{(R 0)}-\sqrt{3} \lambda^{(15)}}{\sqrt{5}}\right)$

$$
\mathcal{G}=2\left(\begin{array}{cccccc}
m & 0 & \sqrt{6} \lambda \omega & \frac{i \eta \bar{\sigma}}{\sqrt{2}} & \frac{-i \eta \sigma}{\sqrt{2}} & 0 \\
0 & m+2 \lambda a & 2 \sqrt{2} \lambda \omega & i \eta \bar{\sigma} \sqrt{\frac{3}{2}} & -i \eta \sigma \sqrt{\frac{3}{2}} & 0 \\
\sqrt{6} \lambda \omega & 2 \sqrt{2} \lambda \omega & m+\lambda(p+2 a) & -i \eta \sqrt{3} \bar{\sigma} & i \sqrt{3} \eta \sigma & 0 \\
\frac{i \eta \bar{\sigma}}{\sqrt{2}} & i \eta \bar{\sigma} \sqrt{\frac{3}{2}} & -i \eta \sqrt{3} \bar{\sigma} & 0 & M+\eta(p+3 a-6 \omega) & \frac{\sqrt{5} g \sigma^{*}}{2} \\
\frac{-i \eta \sigma}{\sqrt{2}} & -i \eta \sigma \sqrt{\frac{3}{2}} & i \eta \sqrt{3} \sigma & M+\eta(p+3 a-6 \omega) & 0 & \frac{\sqrt{5} g \bar{\sigma}^{*}}{2} \\
0 & 0 & 0 & \frac{\sqrt{5} g \sigma^{*}}{2} & \frac{\sqrt{5} g \bar{\sigma}^{*}}{2} & 0
\end{array}\right)
$$


f) $[1,2,-1]\left(\bar{h}_{1}, \bar{h}_{2}, \bar{h}_{3}, \bar{h}_{4}, \bar{h}_{5}, \bar{h}_{6}\right) \oplus[1,2,1]\left(h_{1}, h_{2}, h_{3}, h_{4}, h_{5}, h_{6}\right) \equiv\left(H_{\dot{2}}^{\alpha}, \bar{\Sigma}_{\dot{2}}^{(15) \alpha}\right.$, $\left.\Sigma_{\dot{2}}^{(15) \alpha}, \frac{\Phi_{44}^{\dot{\alpha} \alpha}}{\sqrt{2}}, \Theta_{\dot{2}}^{\alpha}, \Theta_{\dot{2}}^{(15) \alpha}\right) \oplus\left(H_{\alpha \dot{1}}, \bar{\Sigma}_{\alpha \dot{1}}^{(15)}, \Sigma_{\alpha \dot{1}}^{(15)}, \frac{\Phi_{\alpha}^{44 \dot{1}}}{\sqrt{2}}, \Theta_{\alpha \dot{1}}, \Theta_{\alpha \dot{1}}^{(15)}\right)$

$$
\mathcal{H}=\left(\begin{array}{cccccc}
-M_{H} & \bar{\gamma} \sqrt{3}(\omega-a) & -\gamma \sqrt{3}(\omega+a) & -\bar{\gamma} \bar{\sigma} & k p & -\sqrt{3} i k \omega \\
-\bar{\gamma} \sqrt{3}(\omega+a) & 0 & -(2 M+4 \eta(a+\omega)) & 0 & -\sqrt{3} \bar{\zeta} \omega & i(p+2 \omega) \bar{\zeta} \\
\gamma \sqrt{3}(\omega-a) & -(2 M+4 \eta(a-\omega)) & 0 & -2 \eta \bar{\sigma} \sqrt{3} & \sqrt{3} \zeta \omega & -i(p-2 \omega) \zeta \\
-\sigma \gamma & -2 \eta \sigma \sqrt{3} & 0 & -2 m+6 \lambda(\omega-a) & \zeta \sigma & \sqrt{3} i \zeta \sigma \\
p k & \sqrt{3} \bar{\zeta} \omega & -\sqrt{3} \omega \zeta & \bar{\zeta} \bar{\sigma} & -m_{\Theta} & \frac{\rho}{\sqrt{3}} i \omega \\
\sqrt{3} i k \omega & i(p-2 \omega) \bar{\zeta} & -i(p+2 \omega) \zeta & -\sqrt{3} i \bar{\zeta} \bar{\sigma} & -\frac{\rho}{\sqrt{3}} i \omega & -m_{\Theta}-\frac{2 \rho}{3} a
\end{array}\right)
$$

The above matrix is to be diagonalized after imposing the fine tuning condition $\operatorname{Det} \mathcal{H}=0$ to keep one pair of doublets light.

g) $\left[\overline{3}, 1,-\frac{4}{3}\right]\left(\bar{J}_{1}, \bar{J}_{2}, \bar{J}_{3}, \bar{J}_{4}, \bar{J}_{5}\right) \oplus\left[3,1, \frac{4}{3}\right]\left(J_{1}, J_{2}, J_{3}, J_{4}, J_{5}\right)$

$$
\equiv\left(\Sigma_{(R-)}^{\bar{\mu} 4}, \phi_{4}^{\bar{\mu}}, \phi_{4}^{\bar{\mu}(R 0)}, \lambda_{4}^{\bar{\mu}}, \Theta_{(R-)}^{\bar{\mu} 4}\right) \oplus\left(\bar{\Sigma}_{\bar{\mu} 4(R+)}, \phi_{\bar{\mu}}^{4}, \phi_{\bar{\mu}(R 0)}^{4}, \lambda_{\bar{\mu}}^{4}, \Theta_{\bar{\mu} 4}^{(R+)}\right)
$$

$\mathcal{J}=\left(\begin{array}{ccccc}2(M+\eta(a+p-2 \omega)) & -2 \eta \bar{\sigma} & 2 \sqrt{2} \eta \bar{\sigma} & -i g \sqrt{2} \sigma^{*} & 2 \zeta(a-2 \omega) \\ 2 \eta \sigma & -2(m+\lambda a) & -2 \sqrt{2} \lambda \omega & -2 i g \sqrt{2} a^{*} & -\sigma \zeta \\ -2 \sqrt{2} \eta \sigma & -2 \sqrt{2} \lambda \omega & -2(m+\lambda(a+p)) & -4 i g \omega^{*} & \sqrt{2} \sigma \zeta \\ -i g \sqrt{2} \bar{\sigma}^{*} & 2 \sqrt{2} i g a^{*} & 4 i g \omega^{*} & 0 & 0 \\ 2 \bar{\zeta}(a-2 \omega) & \bar{\sigma} \bar{\zeta} & -\sqrt{2} \bar{\sigma} \bar{\zeta} & 0 & m_{\Theta}+\frac{\rho}{3}(p-2 \omega)\end{array}\right)$

h) $\left[\overline{3}, 1, \frac{8}{3}\right]\left(\bar{K}_{1}, \bar{K}_{2}\right) \oplus\left[3,1,-\frac{8}{3}\right]\left(K_{1}, K_{2}, \quad\right) \equiv\left(\Sigma_{(R+)}^{\bar{\mu} 4}, \Theta_{(R+)}^{\bar{\mu} 4}\right) \oplus\left(\bar{\Sigma}_{\bar{\mu} 4(R-)}, \Theta_{\bar{\mu} 4(R-)}\right)$

$$
\mathcal{K}=\left(\begin{array}{cc}
2(M+\eta(a+p+2 \omega)) & 2 \zeta(a+2 \omega) \\
2 \bar{\zeta}(a+2 \omega) & m_{\Theta}+\frac{\rho}{3}(p+2 \omega)
\end{array}\right)
$$

i) $\left[\overline{6}, 1,-\frac{2}{3}\right]\left(\bar{L}_{1}, \bar{L}_{2}\right) \oplus\left[6,1, \frac{2}{3}\right]\left(L_{1}, L_{2}, \quad\right) \equiv\left(\Sigma_{(R 0)}^{\prime \bar{\mu} \bar{\nu}(s)}, \quad \Theta^{\prime \bar{\mu} \bar{\nu}(s)}\right) \oplus$ $\left(\bar{\Sigma}_{\bar{\mu} \bar{\nu}(s)(R 0)}^{\prime}, \quad \Theta_{\bar{\mu} \bar{\nu}(s)}^{\prime}\right)$

$$
\mathcal{L}=\left(\begin{array}{cc}
2(M+\eta(p-a)) & -2 i \zeta \omega \\
2 i \bar{\zeta} \omega & m_{\Theta}-\frac{\rho}{3} a
\end{array}\right)
$$

j) $\left[\overline{3}, 3, \frac{2}{3}\right]\left(\bar{P}_{1}, \bar{P}_{2}\right) \oplus\left[3,3,-\frac{2}{3}\right]\left(P_{1}, P_{2}, \quad\right) \equiv\left(\vec{\Sigma}_{(L)}^{\bar{\mu} 4}, \vec{\Theta}_{(L)}^{\bar{\mu} 4}\right) \oplus\left(\vec{\Sigma}_{\bar{\mu} 4(L)}, \vec{\Theta}_{\bar{\mu} 4(L)}\right)$

$$
\mathcal{P}=\left(\begin{array}{cc}
2(M+\eta(a-p)) & 2 a \bar{\zeta} \\
2 a \zeta & m_{\Theta}-\frac{\rho}{3} p
\end{array}\right)
$$

k) $[8,1,0]\left(R_{1}, R_{2}\right) \equiv\left(\hat{\phi}_{\bar{\mu}}^{\bar{\nu}}, \hat{\phi}_{\bar{\mu}(R 0)}^{\bar{\nu}}\right)$

$$
\mathcal{R}=2\left(\begin{array}{cc}
(m-\lambda a) & -\sqrt{2} \lambda \omega \\
-\sqrt{2} \lambda \omega & m+\lambda(p-a)
\end{array}\right)
$$




$$
\begin{aligned}
& \text { 1) }\left[\overline{3}, 1, \frac{2}{3}\right]\left(\bar{t}_{1}, \bar{t}_{2}, \bar{t}_{3}, \bar{t}_{4}, \bar{t}_{5}, \bar{t}_{6}, \bar{t}_{7}\right) \oplus\left[3,1,-\frac{2}{3}\right]\left(t_{1}, t_{2}, t_{3}, t_{4}, t_{5}, t_{6}, t_{7}\right) \equiv\left(H^{\bar{\mu} 4}, \bar{\Sigma}_{(a)}^{\bar{\mu} 4},\right. \\
& \left.\Sigma_{(a)}^{\bar{\mu} 4}, \Sigma_{(R 0)}^{\bar{\mu} 4}, \Phi_{4(R+)}^{\bar{\mu}}, \Theta^{\bar{\mu} 4(s)}, \Theta_{(R 0)}^{\bar{\mu} 4}\right) \oplus\left(H_{\bar{\mu} 4}, \bar{\Sigma}_{\bar{\mu} 4(a)}, \Sigma_{\bar{\mu} 4(a)}, \bar{\Sigma}_{\bar{\mu} 4(R 0)}, \Phi_{\bar{\mu}(R-)}^{4}, \Theta_{\bar{\mu} 4(s)}, \Theta_{\bar{\mu} 4(R 0)}\right) \\
& \mathcal{T}=\left(\begin{array}{ccccccc}
M_{H} & \bar{\gamma}(a+p) & \gamma(p-a) & 2 \sqrt{2} i \omega \bar{\gamma} & i \bar{\sigma} \bar{\gamma} & \sqrt{2} k a & \sqrt{2} i k \omega \\
\bar{\gamma}(p-a) & 0 & 2 M & 0 & 0 & \sqrt{2} a \bar{\zeta} & \sqrt{2} i \omega \bar{\zeta} \\
\gamma(p+a) & 2 M & 0 & 4 \sqrt{2} i \omega \eta & 2 i \eta \bar{\sigma} & -\sqrt{2} a \zeta & \sqrt{2} i \omega \zeta \\
-2 \sqrt{2} i \omega \gamma & -4 \sqrt{2} i \omega \eta & 0 & 2 M+2 \eta p+2 \eta a & -2 \sqrt{2} \eta \bar{\sigma} & 2 i \omega \zeta & 2 \zeta a \\
i \sigma \gamma & 2 i \eta \sigma & 0 & 2 \sqrt{2} \eta \sigma & -2 m-2 \lambda(a+p-4 \omega) & \sqrt{2} i \sigma \zeta & -\sqrt{2} \zeta \sigma \\
\sqrt{2} k a & -\sqrt{2} a \bar{\zeta} & \sqrt{2} a \zeta & -2 i \bar{\zeta} \omega & \sqrt{2} i \bar{\zeta} \bar{\sigma} & m_{\Theta}+\frac{\rho}{3} a & -\frac{2 i}{3} \rho \omega \\
-\sqrt{2} i k \omega & -\sqrt{2} i \omega \bar{\zeta} & -\sqrt{2} i \omega \zeta & 2 \zeta a & \sqrt{2} \bar{\sigma} \bar{\zeta} & \frac{2 i}{3} \rho \omega & m_{\Theta}+\frac{\rho}{3} p
\end{array}\right)
\end{aligned}
$$

m) $\left[3,2, \frac{5}{3}\right]\left(\bar{X}_{1}, \bar{X}_{2}, \bar{X}_{3}\right) \oplus\left[3,2,-\frac{5}{3}\right]\left(X_{1}, X_{2}, X_{3}\right)$

$$
\begin{gathered}
\equiv\left(\phi_{\alpha \dot{1}}^{(s) \bar{\mu} 4}, \phi_{\alpha \dot{1}}^{(a) \bar{\mu} 4}, \lambda_{\alpha \dot{1}}^{\bar{\mu} 4}\right) \oplus\left(\phi_{\bar{\mu} 4 \alpha \dot{2}}^{(s)}, \phi_{\bar{\mu} 4 \alpha \dot{2}}^{(a)}, \lambda_{\bar{\mu} 4 \alpha \dot{2}}\right) \\
\mathcal{X}=\left(\begin{array}{ccc}
2(m+\lambda(a+\omega)) & -2 \sqrt{2} \lambda \omega & -2 g\left(a^{*}+\omega^{*}\right) \\
-2 \sqrt{2} \lambda \omega & 2(m+\lambda \omega) & \sqrt{2} g\left(\omega^{*}+p^{*}\right) \\
-2 g\left(a^{*}+\omega^{*}\right) & \sqrt{2} g\left(\omega^{*}+p^{*}\right) & 0
\end{array}\right)
\end{gathered}
$$

\section{Susy Threshold Corrections}

A complete evaluation of 1-loop radiative corrections for the MSSM in the absence of generation mixing is available in 60] which is an invaluable and essential reference for our work. The approximations(to their own exact 1-loop results) of [60] are however used in our actual calculation with the modification that for the down type $\left(T_{3 L}=-1 / 2\right)$ fermions we also add the Bino correction given in 30 to the chargino and gluino corrections considered in the approximation advocated in [60]; in any case difference is small. The two give closely similar results. As in [60], we use the gluon and squark-gluino correction to correct the top quark Yukawa from that given by the pole mass and apply the squark-gluino corrections to the running up and charm quark Yukawa couplings since these corrections are not insignificant either( see Tables 5,11).

We next summarize the approximations to the 1-loop threshold corrections computed in [60]. The corrections are computed in the absence of generation mixing. The dominant corrections to the top quark Yukawa are the gluon and squark-gluino loops. To estimate the leading corrections to the top quark Yukawa $y_{t}$, they set the electroweak couplings and the Yukawas to zero : $g=g^{\prime}=$ 0 and $y_{t}=y_{b}=0$ and simplify the resulting expressions by setting the external momenta $p^{2}=0$ because $m_{t}$ is much smaller than a typical squark or gluino mass. In that limit, the physical top quark mass is given by

$$
m_{t}=\hat{m}_{t}(Q)\left[1+\frac{\Delta m_{t}}{\hat{m}_{t}}\right]
$$

where $\hat{m}_{t}$ is the MSSM tree level mass and $\Delta m_{t}$ is the one-loop correction due to (heavy) sparticles computed in the effective MSSM. The one-loop correction $\frac{\Delta m_{t}}{\hat{m}_{t}}$ receives two important contributions. The first is the finite gluon correction

$$
\left(\frac{\Delta m_{t}}{m_{t}}\right)^{t g}=\frac{g_{3}^{2}}{12 \pi^{2}}\left[3 \ln \left(\frac{M_{Z}^{2}}{m_{t}^{2}}\right)+5\right] .
$$

The renormalization scale is always chosen to be $M_{Z}$. The second correction comes from the top 
$\operatorname{squark}(\tilde{t}) / \operatorname{gluino}(\tilde{g})$ loops,

$$
\begin{aligned}
\left(\frac{\Delta m_{t}}{m_{t}}\right)^{\tilde{t} \tilde{g}}= & -\frac{g_{3}^{2}}{12 \pi^{2}}\left\{B_{1}\left(0, m_{\tilde{g}}, m_{\tilde{t}_{1}}\right)+B_{1}\left(0, m_{\tilde{g}}, m_{\tilde{t}_{2}}\right)\right. \\
& \left.-\sin \left(2 \theta_{t}\right)\left(\frac{m_{\tilde{g}}}{m_{t}}\right)\left[B_{0}\left(0, m_{\tilde{g}}, m_{\tilde{t}_{1}}\right)-B_{0}\left(0, m_{\tilde{g}}, m_{\tilde{t}_{2}}\right)\right]\right\}
\end{aligned}
$$

where $\theta_{t}$ is the top-squark LR mixing angle (for any sfermion mixing angle $\theta_{f}: \tan 2 \theta_{\tilde{f}}=$ $2\left(M_{L R}^{2} /\left(M_{L L}^{2}-M_{R R}^{2}\right)\right)_{f}$ and the approximate forms of the loop functions are :

$$
\begin{gathered}
B_{0}\left(0, m_{1}, m_{2}\right)=-\ln \left(\frac{M^{2}}{Q^{2}}\right)+1+\frac{m^{2}}{m^{2}-M^{2}} \ln \left(\frac{M^{2}}{m^{2}}\right), \\
B_{1}\left(0, m_{1}, m_{2}\right)=\frac{1}{2}\left[-\ln \left(\frac{M^{2}}{Q^{2}}\right)+\frac{1}{2}+\frac{1}{1-x}+\frac{\ln x}{(1-x)^{2}}-\theta(1-x) \ln x\right],
\end{gathered}
$$

with $M=\max \left(m_{1}, m_{2}\right), m=\min \left(m_{1}, m_{2}\right)$, and $x=m_{2}^{2} / m_{1}^{2}$.

For the bottom quark it is appropriate to use the running mass at $Q=M_{Z}$ and thus to absorb the gluon corrections in the running bottom quark mass. Taking the bottom quark pole mass $m_{b}=4.9 \mathrm{GeV}$, and $\alpha_{s}\left(M_{Z}\right)=0.118$, they find the standard-model $\overline{\mathrm{DR}}$ value $\hat{m}_{b}\left(M_{Z}\right)^{\mathrm{SM}}=2.92$ $\mathrm{GeV}$. See [55] for a recent review of accurate RG corrected fermion Yukawas.

As in the case of the top quark we then interpret the relation between the SM and MSSM masses(Yukawas) as :

$$
\hat{m}_{b}\left(M_{Z}\right)^{\mathrm{SM}}=\hat{m}_{b}\left(M_{Z}\right)\left[1+\left(\frac{\Delta m_{b}}{\hat{m}_{b}}\right)^{\text {massive }}\right] .
$$

instead of the (perturbatively) equivalent form quoted in [60]

$$
\hat{m}_{b}\left(M_{Z}\right)=\hat{m}_{b}\left(M_{Z}\right)^{\mathrm{SM}}\left[1-\left(\frac{\Delta m_{b}}{m_{b}}\right)^{\text {massive }}\right] .
$$

This is appropriate since the radiative corrections should be calculated consistently in terms of the MSSM couplings. Ignoring the small $W, Z$, Higgs, and neutralino contributions leaves the squark/gluino and squark/chargino loops :

$$
\left(\frac{\Delta m_{b}}{m_{b}}\right)^{\text {massive }}=\left(\frac{\Delta m_{b}}{m_{b}}\right)^{\tilde{b} \tilde{g}}+\left(\frac{\Delta m_{b}}{m_{b}}\right)^{\tilde{t} \tilde{\chi}^{+}}
$$

The squark/gluino contribution is again given by (C.3), with the substitution $t \rightarrow b$. To approximate the squark/chargino contribution, they set $g=g^{\prime}=y_{b}=y_{t}=0$, except for terms that are enhanced by the Higgsino mass parameter $\mu$ or by $\tan \beta$. The chargino masses are set to the Wino mass $M_{2}$ and $\mu$, respectively (this is an excellent approximation in all the parameter sets we will actually find since $\left.\mu>>M_{2}, m_{t}\right)$. In this case squark/chargino loops give rise to the following terms for the down quarks(with similar formulae also for down type charged leptons)

$$
\begin{aligned}
& \left(\frac{\Delta m_{b}}{m_{b}}\right)^{\tilde{t} \tilde{\chi}^{+}}=\frac{y_{t}^{2}}{16 \pi^{2}} \mu \frac{A_{t}^{0} \tan \beta-\mu}{m_{\tilde{t}_{2}}^{2}-m_{\tilde{t}_{1}}^{2}}\left[B_{0}\left(0, \mu, m_{\tilde{t}_{1}}\right)-B_{0}\left(0, \mu, m_{\tilde{t}_{2}}\right)\right] \\
& -\frac{g^{2}}{16 \pi^{2}}\left\{\frac{\mu M_{2} \tan \beta}{\mu^{2}-M_{2}^{2}}\left[c_{t}^{2} B_{0}\left(0, M_{2}, m_{\tilde{t}_{1}}\right)+s_{t}^{2} B_{0}\left(0, M_{2}, m_{\tilde{t}_{2}}\right)\right]\right. \\
& \left.+\left(\mu \leftrightarrow M_{2}\right)\right\}
\end{aligned}
$$


where $B_{0}\left(0, m_{1}, m_{2}\right)$ is defined in (C.4 $)$, and $c_{t}\left(s_{t}\right)$ is $\cos \theta_{t}\left(\sin \theta_{t}\right)$ and we have changed the sign of $\mu$ since our convention is opposite to that of [60].

The same formulae, mutatis mutandis, can be used for the down and strange quarks and, dropping the gluino correction and neutrino-Dirac coupling, for the $T_{3 L}=-1 / 2$ leptons. In a slight improvement we also add the Bino corrections for the $T_{3 L}=-1 / 2$ fermions taken from the formulae quoted in [30] :

$$
\Delta m_{i} /\left(m_{i} \tan \beta\right)=\frac{1}{16 \pi^{2}}\left[\frac{g^{\prime 2}}{6} \frac{M_{1}}{\mu}\left(H_{2}\left(v_{\tilde{Q}_{i}}, x_{1}\right)+2 H_{2}\left(v_{\tilde{d}_{i}}, x_{1}\right)\right)+\frac{g^{\prime 2}}{9} \frac{\mu}{M_{1}} H_{2}\left(w_{\tilde{Q}_{i}}, w_{\tilde{d}_{i}}\right)\right]
$$

where $u_{\tilde{f}}=m_{\tilde{f}}^{2} / M_{3}^{2}, v_{\tilde{f}}=m_{\tilde{f}}^{2} / \mu^{2}, w_{\tilde{f}}=m_{\tilde{f}}^{2} / M_{1}^{2}, x_{1}=M_{1}^{2} / \mu^{2}$ and $x_{2}=M_{2}^{2} / \mu^{2}$ for $i=d, s, b$. All mass parameters are assumed to be real. $H_{2}$ is defined as

$$
H_{2}(x, y)=\frac{x \ln x}{(1-x)(x-y)}+\frac{y \ln y}{(1-y)(y-x)} .
$$

$H_{2}$ is negative for positive $x$ and $y$ and $\left|H_{2}\right|$ is maximal, if its arguments are minimal, and vice versa.

The main point to note is that due to large $\tan \beta$, for the $\mathrm{b}$ quark, a large (MSSM Yukawa lowering) gluino correction can be canceled and even overcome by a large $A_{0} y_{t}$ driven chargino correction to a degree that it alleviates the known tension [62] between the value of $\hat{m}_{b}^{S M}$ from experiment and the value $\hat{m}_{b}^{M S S M}$ required by $\mathrm{SO}(10)$ grand unification. The same is not true for the down and strange quark due to their small Yukawas, so that for them the gluino correction serves just to lower the SM Yukawa couplings as needed to match the MSSM/GUT values. Conversely for the charged leptons there are no gluino corrections. The structure of the threshold corrections is thus just what is needed to alleviate the problems that arise in $\mathrm{SO}(10)$ Yukawa unification including those specific to the NMSGUT. It is is easy to check that the down and strange Yukawas can be significantly lowered only by the gluino correction with a large positive value of the ratio $\mu / M_{3}$. There is a preference for lower values of squark masses for the first two generations. On the other hand for the third generation one has the opposite problem : the desired value of $\hat{y}_{b}^{M S S M}\left(M_{Z}\right)$ corresponds to $\hat{m}_{b}^{S M}\left(M_{Z}\right)$ at least as large as $3.15 \mathrm{GeV}$ whereas the actual central value is about $2.9 \pm .2 \mathrm{GeV}$. Thus one needs to countervail against the gluino correction and a large value of $A_{t}$ can accomplish this. In this paper we have confined ourselves to searches where $\mu$ is real and positive, thus $A_{0}$ emerges negative and large. Although we allow the initial gaugino mass parameter at $M_{X}$ to be negative (and negative $m_{1 / 2}$ values are indeed chosen by the search algorithm: see Tables 3,9) the low energy values are positive. Moreover note that the large values of $A_{0}$ used cause the gaugino mass ratios at $M_{Z}$ to vary considerably from the approximately 1:2:7 (i.e. $1: g_{2}^{2} / g_{1}^{2}: g_{3}^{2} / g_{1}^{2}$ ) ratio expected on the basis of one-loop RG flow down to $M_{Z}$ from universal gaugino mass boundary condition we use at $M_{X}$. The range of possibilities for these ratios that we actually encounter in our work -given that $A_{0}, m_{\tilde{f}}$ are always large - is so different from that encountered at small values of these parameters that the oft advocated abandonment of the universal gaugino mass condition at $M_{X}$ to achieve such diversity seems ill advised till it has indeed been verified that suitable large values of $A_{0}$ etc will not serve equally well. Finally we note that the third generation sfermion masses are driven to large values by the large negative values of the Higgs mass-squared parameters which occur (already at one loop) multiplied with the Yukawa couplings squared in the RGE for sfermion masses squared. This enabling feature is discussed more completely in 38. (Appendix B).

For completeness, we also mention the 1-loop electroweak symmetry breaking conditions that 
we imposed:

$$
\begin{aligned}
\mu^{2} & =\frac{1}{2}\left[\tan 2 \beta\left(\bar{m}_{H_{2}}^{2} \tan \beta-\bar{m}_{H_{1}}^{2} \cot \beta\right)-M_{Z}^{2}\right] \\
B & =\frac{1}{2}\left(\tan 2 \beta\left(\bar{m}_{H_{2}}^{2}-\bar{m}_{H_{1}}^{2}\right)-M_{Z}^{2} \sin 2 \beta\right)
\end{aligned}
$$

where $H_{2} \equiv H, H_{1} \equiv \bar{H}, \bar{m}_{H_{1}}^{2}=m_{H_{1}}^{2}-t_{1} / v_{1}, \bar{m}_{H_{2}}^{2}=m_{H_{2}}^{2}-t_{2} / v_{2}$ and $v_{1,2}, t_{1,2}$ are vevs and tadpoles(calculated using a subroutine taken from the SPheno Susy spectrum code 64 based on the formulae of [60]) of the effective potential. Note that the Higgs scalar masses we quote are also output from SPHENO [64] subroutine that however uses the method of [65]. Thus the pseudo-scalar mass $M_{A}$ is computed from the one-loop corrected value of $B$ in eqn.(C.12) above using the tree level relation $\left(M_{A}^{2}=2 B / \sin 2 \beta\right)$ while the Higgs scalar masses are computed using the effective potential masses. The 1-loop pole masses for these particles require also computation of the self-energies [60] which are not included here. Note that we use the interpolation formula suggested in 60] for the electroweak vev in the MSSM:

$$
v=\left[248.6+0.9 \operatorname{Ln} \frac{M_{\tilde{q}}}{M_{Z}}\right] G e V \quad ; \quad M_{\tilde{q}}^{2}=4 m_{\frac{1}{2}}^{2}+m_{\tilde{f}}^{2}
$$




\begin{tabular}{|c|c|c|c|}
\hline Appendix D & Two & Example & fits \\
\hline Parameter & Value & $\begin{array}{c}\text { Field } \\
{[S U(3), S U(2), Y]}\end{array}$ & $\begin{array}{c}\text { Masses } \\
\left.\text { (Units of } 10^{16} \mathrm{Gev}\right)\end{array}$ \\
\hline$\chi_{X}$ & 0.0338 & $A[1,1,4]$ & 19.73 \\
\hline$\chi_{Z}$ & 0.0149 & $B[6,2,5 / 3]$ & 0.0224 \\
\hline$h_{11} / 10^{-6}$ & -0.3965 & $C[8,2,1]$ & $2.59,18.50,18.98$ \\
\hline$h_{22} / 10^{-4}$ & 9.9684 & $D[3,2,7 / 3]$ & $0.57,5.84,8.41$ \\
\hline$h_{33}$ & -0.3872 & $E[3,2,1 / 3]$ & $0.03,1.03,1.03$ \\
\hline$f_{11} / 10^{-6}$ & $-0.2749-0.1421 i$ & & $1.729,9.94,11.97$ \\
\hline$f_{12} / 10^{-6}$ & $2.0663+0.4203 i$ & $F[1,1,2]$ & $0.34,0.34$ \\
\hline$f_{13} / 10^{-5}$ & $1.0541-0.5949 i$ & & $1.95,10.08$ \\
\hline$f_{22} / 10^{-5}$ & $-0.9652-0.6818 i$ & $G[1,1,0]$ & $0.004,0.04,0.05$ \\
\hline$f_{23} / 10^{-4}$ & $0.6791-1.3076 i$ & & $0.052,1.23,1.25$ \\
\hline$f_{33} / 10^{-3}$ & $2.6506-1.3235 i$ & $h[1,2,1]$ & $0.234,2.52,3.38$ \\
\hline$g_{12} / 10^{-3}$ & $0.5392+0.1579 i$ & & $4.83,9.75$ \\
\hline$g_{13} / 10^{-3}$ & $2.5557-4.6432 i$ & $I[3,1,10 / 3]$ & 0.07 \\
\hline$g_{23} / 10^{-2}$ & $1.0256+3.9768 i$ & $J[3,1,4 / 3]$ & $0.057,0.75,0.75$ \\
\hline$\lambda / 10^{-2}$ & $-1.9313-0.2227 i$ & & $3.34,13.47$ \\
\hline$\eta$ & $-5.2157-3.1585 i$ & $K[3,1,8 / 3]$ & $3.31,17.44$ \\
\hline$\rho$ & $-0.7161-1.5279 i$ & $L[6,1,2 / 3]$ & $3.06,25.18$ \\
\hline$k$ & $-0.6362-0.0019 i$ & $M[6,1,8 / 3]$ & 26.49 \\
\hline$\zeta$ & $-0.9531-0.3806 i$ & $N[6,1,4 / 3]$ & 24.23 \\
\hline $\bar{\zeta}$ & $1.4806+1.2424 i$ & $O[1,3,2]$ & 24.62 \\
\hline$m / 10^{16} \mathrm{GeV}$ & 0.00306 & $P[3,3,2 / 3]$ & $2.37,21.08$ \\
\hline$m_{\Theta} / 10^{16} \mathrm{GeV}$ & $-2.805 e^{-i \operatorname{Arg}(\lambda)}$ & $Q[8,3,0]$ & 0.054 \\
\hline$\gamma$ & 3.18293 & $R[8,1,0]$ & $0.02,0.06$ \\
\hline $\bar{\gamma}$ & 0.5553 & $S[1,3,0]$ & 0.0761 \\
\hline$x$ & $0.6944+0.5020 i$ & $t[3,1,2 / 3]$ & $0.04,0.78,2.70,3.33$ \\
\hline$\Delta_{X}$ & -0.30 & & $12.75,13.63,93.77$ \\
\hline$\Delta_{G}$ & 5.350 & $U[3,3,4 / 3]$ & 0.064 \\
\hline$\Delta \alpha_{3}\left(M_{Z}\right)$ & -0.016 & $V[1,2,3]$ & 0.055 \\
\hline$\left\{M^{\nu^{c}} / 10^{11} \mathrm{GeV}\right\}$ & $0.0108,0.33,107.72$ & $W[6,3,2 / 3]$ & 23.51 \\
\hline$\left\{M_{I I}^{\nu} / 10^{-7} \mathrm{eV}\right\}$ & $0.0109,0.33,108.83$ & $X[3,2,5 / 3]$ & $0.019,1.189,1.189$ \\
\hline$M_{\nu}(m e V)$ & $2.2845,7.62,41.82$ & $Y[6,2,1 / 3]$ & 0.03 \\
\hline$\{$ Evals $[\mathrm{f}]\} / 10^{-5} \mathrm{eV}$ & $0.02972,0.903,297.00$ & $Z[8,1,2]$ & 0.06 \\
\hline Soft parameters & $\mathrm{m}_{\frac{1}{2}}=-445.837$ & $\mathrm{~m}_{0}=7018.005$ & $\mathrm{~A}_{0}=-2.8605 \times 10^{5}$ \\
\hline at $M_{X}^{0}$ & $\mu \stackrel{2}{=} 2.2652 \times 10^{5}$ & $\mathrm{~B}=-3.5698 \times 10^{10}$ & $\tan \beta=50.0000$ \\
\hline & $\mathrm{M}_{\overline{\mathrm{H}}}^{2}=-3.6296 \times 10^{10}$ & $\mathrm{M}_{\mathrm{H}}^{2}=-3.4956 \times 10^{10}$ & $\mathrm{R}_{\underline{\underline{b} \tau}}=0.9530$ \\
\hline $\operatorname{Max}\left(\left|L_{A B C D}\right|,\left|R_{A B C D}\right|\right)$ & $2.3854 \times 10^{-18} \mathrm{GeV}^{-1}$ & & \\
\hline
\end{tabular}

Table 3: Case 1 : Column 1 contains values of the NMSGUT-SUGRY-NUHM parameters at $M_{X}$ derived from an accurate fit to all 18 fermion data and compatible with RG constraints. Unification parameters and mass spectrum of superheavy and superlight fields are also given. The values of $\mu\left(M_{X}^{0}\right), B\left(M_{X}^{0}\right)$ are determined by RG evolution from $M_{Z}$ to $M_{X}^{0}$ of the values determined by the EWRSB conditions. 


\begin{tabular}{|c|c|c|c|c|}
\hline & & & & \\
Parameter & Target $=\bar{O}_{i}$ & Uncert. $=\delta_{i}$ & Achieved $=O_{i}$ & Pull $=\left(O_{i}-\bar{O}_{i}\right) / \delta_{i}$ \\
\hline$y_{u} / 10^{-6}$ & 2.044938 & 0.781166 & 2.045688 & 0.000960 \\
$y_{c} / 10^{-3}$ & 0.996830 & 0.164477 & 0.997657 & 0.005028 \\
$y_{t}$ & 0.378790 & 0.015152 & 0.378779 & -0.000722 \\
$y_{d} / 10^{-5}$ & 7.128042 & 4.155648 & 7.073763 & -0.013061 \\
$y_{s} / 10^{-3}$ & 1.351200 & 0.637766 & 1.368823 & 0.027632 \\
$y_{b}$ & 0.540954 & 0.280755 & 0.542439 & 0.005288 \\
$y_{e} / 10^{-4}$ & 1.276823 & 0.191523 & 1.276139 & -0.003569 \\
$y_{\mu} / 10^{-2}$ & 2.589599 & 0.388440 & 2.589488 & -0.000285 \\
$y_{\tau}$ & 0.566903 & 0.107712 & 0.565812 & -0.010128 \\
$\sin _{12}^{q} \theta_{12}^{q} / 10^{-4}$ & 0.2210 & 0.001600 & 0.2210 & 0.0008 \\
$\sin \theta_{13}^{q} \sin _{23} / 10^{-3}$ & 38.6321 & 5.000000 & 28.6231 & -0.0018 \\
$\delta^{q}$ & 60.0208 & 1.300000 & 33.6848 & -0.0036 \\
$\left(m_{12}^{2}\right) / 10^{-5}(e V)^{2}$ & 5.2894 & 0.560672 & 59.9598 & -0.0044 \\
$\left(m_{23}^{2}\right) / 10^{-3}(e V)^{2}$ & 1.6906 & 0.338115 & 1.6906 & 0.0002 \\
$\sin ^{2} \theta_{12}^{L}$ & 0.2934 & 0.058683 & 0.2934 & 0.0001 \\
$\sin ^{2} \theta_{23}^{L}$ & 0.4585 & 0.137561 & 0.4585 & 0.0000 \\
$\sin ^{2} \theta_{13}^{L}$ & 0.0249 & 0.019000 & 0.0249 & -0.0003 \\
\hline$\alpha_{1}$ & $0.3453-0.0000 i$ & $\bar{\alpha}_{1}$ & $0.4938-0.0000 i$ & \\
$\alpha_{2}$ & $0.1119-0.0124 i$ & $\bar{\alpha}_{2}$ & $0.3341+0.0788 i$ & \\
$\alpha_{3}$ & $0.0721-0.0878 i$ & $\bar{\alpha}_{3}$ & $0.0730-0.1041 i$ & \\
$\alpha_{4}$ & $-0.6519+0.6401 i$ & $\bar{\alpha}_{4}$ & $0.2867+0.4862 i$ & \\
$\alpha_{5}$ & $-0.0217-0.0724 i$ & $\bar{\alpha}_{5}$ & $-0.0646-0.1725 i$ & \\
$\alpha_{6}$ & $-0.1213-0.0089 i$ & $\bar{\alpha}_{6}$ & $-0.5050-0.1211 i$ & \\
\hline
\end{tabular}

Table 4: Example 1: with $\chi_{X}=\sqrt{\sum_{i=1}^{17}\left(O_{i}-\bar{O}_{i}\right)^{2} / \delta_{i}^{2}}=0.0338$. Target values, at $M_{X}^{0}$ of the fermion Yukawa couplings and mixing parameters, together with the estimated uncertainties, achieved values and pulls. The eigenvalues of the wavefunction renormalization increment matrices $\Delta_{i}$ for fermion lines and the factors for Higgs lines are given, assuming the external Higgs is 10-plet dominated. The Higgs fractions $\alpha_{i}, \bar{\alpha}_{i}$ which control the MSSM fermion Yukawa couplings are also given. Notice the dominance of the first components $\alpha_{1}, \overline{\alpha_{1}}$ consistently with the assumption made. Right-handed neutrino threshold effects have been ignored. We have truncated numbers for display although all calculations are done at double precision. 


\begin{tabular}{|c|c|c|c|}
\hline & & & \\
Parameter & $S M\left(M_{Z}\right)$ & $m^{G U T}\left(M_{Z}\right)$ & $m^{M S S M}=(m+\Delta m)^{G U T}\left(M_{Z}\right)$ \\
\hline$m_{d} / 10^{-3}$ & 2.90000 & 0.61793 & 2.87054 \\
$m_{s} / 10^{-3}$ & 55.00000 & 11.95726 & 55.58066 \\
$m_{b}$ & 3.00000 & 3.24165 & 2.99969 \\
$m_{e} / 10^{-3}$ & 0.48657 & 0.47227 & 0.48588 \\
$m_{\mu}$ & 0.10272 & 0.09579 & 0.10267 \\
$m_{\tau}$ & 1.74624 & 1.73972 & 1.74281 \\
$m_{u} / 10^{-3}$ & 1.27000 & 1.08293 & 1.27036 \\
$m_{c}$ & 0.61900 & 0.52813 & 0.61955 \\
$m_{t}$ & 172.50000 & 146.75487 & 172.43486 \\
\hline
\end{tabular}

Table 5: Example 1: Values of standard model fermion masses in $\mathrm{GeV}$ at $M_{Z}$ compared with the masses obtained from values of GUT derived Yukawa couplings run down from $M_{X}^{0}$ to $M_{Z}$ both before and after threshold corrections. Fit with $\chi_{Z}=\sqrt{\sum_{i=1}^{9}\left(m_{i}^{M S S M}-m_{i}^{S M}\right)^{2} /\left(m_{i}^{M S S M}\right)^{2}}=0.0149$.

\begin{tabular}{|c|c|c|c|}
\hline Parameter & Value & Parameter & Value \\
\hline$M_{1}$ & 223.01 & $M_{\tilde{u}_{1}}$ & 9225.58 \\
$M_{2}$ & 661.94 & $M_{\tilde{u}_{2}}$ & 9222.53 \\
$M_{3}$ & 827.66 & $M_{\tilde{u}_{3}}$ & 29944.82 \\
$M_{\tilde{\bar{l}}_{1}}$ & 2890.72 & $A_{11}^{0(l)}$ & -172061.46 \\
$M_{\tilde{\bar{l}}_{2}}$ & 230.51 & $A_{22}^{0(l)}$ & -171854.14 \\
$M_{\tilde{l}_{3}}$ & 19852.55 & $A_{33}^{0(l)}$ & -103991.62 \\
$M_{\tilde{L}_{1}}$ & 12250.20 & $A_{11}^{0(u)}$ & -209999.77 \\
$M_{\tilde{L}_{2}}$ & 12080.49 & $A_{22}^{0(u)}$ & -209998.40 \\
$M_{\tilde{L}_{3}}$ & 18971.40 & $A_{33}^{0(u)}$ & -106625.21 \\
$M_{\tilde{\bar{d}}_{1}}$ & 4654.53 & $A_{11}^{0(d)}$ & -172245.57 \\
$M_{\tilde{\bar{d}}_{2}}$ & 4651.04 & $A_{22}^{0(d)}$ & -172244.04 \\
$M_{\tilde{\bar{d}}_{3}}$ & 66504.44 & $A_{33}^{0(d)}$ & -59367.97 \\
$M_{\tilde{Q}_{1}}$ & 9877.51 & $\tan \beta$ & 50.00 \\
$M_{\tilde{Q}_{2}}$ & 9875.28 & $\mu\left(M_{Z}\right)$ & 174668.73 \\
$M_{\tilde{Q}_{3}}$ & 51813.13 & $B\left(M_{Z}\right)$ & $5.6389 \times 10^{9}$ \\
$M_{\tilde{H}^{2}}^{2}$ & $-2.9298 \times 10^{10}$ & $M_{H}^{2}$ & $-3.3648 \times 10^{10}$ \\
\hline
\end{tabular}

Table 6: Example 1: Values $(\mathrm{GeV})$ in of the soft Susy parameters at $M_{Z}$ (evolved from the soft SUGRYNUHM parameters at $M_{X}^{0}$ ). The values of soft Susy parameters at $M_{Z}$ determine the Susy threshold corrections to the fermionYukawas. The matching of run down fermion Yukawas in the MSSM to the SM parameters determines soft SUGRY parameters at $M_{X}$. Note the heavier third sgeneration. The values of $\mu\left(M_{Z}\right)$ and the corresponding soft Susy parameter $B\left(M_{Z}\right)=$ $m_{A}^{2} \sin 2 \beta / 2$ are determined by imposing electroweak symmetry breaking conditions. $m_{A}$ is the mass of the CP odd scalar in the Doublet Higgs. The sign of $\mu$ is assumed positive. 


\begin{tabular}{|c|c|}
\hline Field & Mass $(\mathrm{GeV})$ \\
\hline$M_{\tilde{G}}$ & 827.66 \\
$M_{\chi^{ \pm}}$ & $661.94,174668.77$ \\
$M_{\chi^{0}}$ & $223.01,661.94,174668.76,174668.76$ \\
$M_{\tilde{\nu}}$ & $12250.021,12080.306,18971.280$ \\
$M_{\tilde{e}}$ & $2891.07,12250.30,224.01,12080.79,18815.46,20000.51$ \\
$M_{\tilde{u}}$ & $9225.51,9877.36,9222.40,9875.18,29942.71,51814.72$ \\
$M_{\tilde{d}}$ & $4654.61,9877.70,4651.10,9875.48,51808.66,66507.96$ \\
$M_{A}$ & 531090.49 \\
$M_{H^{ \pm}}$ & 531090.49 \\
$M_{H^{0}}$ & 531090.47 \\
$M_{h^{0}}$ & 114.58 \\
\hline
\end{tabular}

Table 7: Example 1:Spectra of supersymmetric partners calculated ignoring generation mixing effects. Inclusion of such effects changes the spectra only marginally. Due to the large values of $\mu, B, A_{0}$. The LSP and light chargino are essentially pure Bino and Wino $\left(\tilde{W}_{ \pm}\right)$. The light gauginos and light Higgs $h^{0}$, are accompanied by a light smuon and sometimes selectron. The rest of the sfermions have multi-TeV masses. The mini-split supersymmetry spectrum and large $\mu, A_{0}$ parameters help avoid problems with FCNC and CCB/UFB instability [38,71. The sfermion masses are ordered by generation not magnitude. This is useful in understanding the spectrum calculated including generation mixing effects

\begin{tabular}{|c|c|}
\hline Field & Mass $(\mathrm{GeV})$ \\
\hline$M_{\tilde{G}}$ & 821.02 \\
$M_{\chi^{ \pm}}$ & $660.62,174947.06$ \\
$M_{\chi^{0}}$ & $222.65,660.62,174947.05,174947.05$ \\
$M_{\tilde{\nu}}$ & $12075.63,12245.38,18876.120$ \\
$M_{\tilde{e}}$ & $324.73,2900.24,12076.12,12245.66,18706.96,19837.44$ \\
$M_{\tilde{u}}$ & $9240.12,9243.22,9860.87,9892.76,29570.81,51588.50$ \\
$M_{\tilde{d}}$ & $4693.25,4696.77,9861.19,9893.10,51582.41,66310.78$ \\
$M_{A}$ & 532268.90 \\
$M_{H^{ \pm}}$ & 532268.91 \\
$M_{H^{0}}$ & 532268.89 \\
$M_{h^{0}}$ & 113.41 \\
\hline
\end{tabular}

Table 8: Example 1: Spectra of supersymmetric partners calculated including generation mixing effects. Inclusion of such effects changes the spectra only marginally. Due to the large values of $\mu, B, A_{0}$. The LSP and light chargino are essentially pure Bino and Wino $\left(\tilde{W}_{ \pm}\right)$. Note that the ordering of the eigenvalues in this table follows their magnitudes, comparison comparison with the previous table is necessary to identify the sfermions 


\begin{tabular}{|c|c|c|c|}
\hline Parameter & Value & $\begin{array}{c}\text { Field } \\
{[S U(3), S U(2), Y]}\end{array}$ & $\begin{array}{c}\text { Masses } \\
\left.\text { (Units of } 10^{16} \mathrm{Gev}\right)\end{array}$ \\
\hline$\overline{\chi_{X}}$ & 0.0528 & $A[1,1,4]$ & 3502.30 \\
\hline$\chi_{Z}$ & 0.0227 & $B[6,2,5 / 3]$ & 0.4053 \\
\hline$h_{11} / 10^{-6}$ & -2.6106 & $C[8,2,1]$ & $162.52,1146.22,1385.40$ \\
\hline$h_{22} / 10^{-4}$ & -8.1592 & $D[3,2,7 / 3]$ & $139.23,2043.80,2350.42$ \\
\hline$h_{33}$ & 0.3114 & $E[3,2,1 / 3]$ & $0.96,150.01,150.01$ \\
\hline$f_{11} / 10^{-6}$ & $-0.0630-0.0424 i$ & & $317.336,670.73,1575.37$ \\
\hline$f_{12} / 10^{-6}$ & $-0.0357-2.0877 i$ & $F[1,1,2]$ & $79.95,79.95$ \\
\hline$f_{13} / 10^{-5}$ & $-0.6349-0.1413 i$ & & $663.71,1826.48$ \\
\hline$f_{22} / 10^{-5}$ & $-10.5738+6.5114 i$ & $G[1,1,0]$ & $0.074,0.69,2.34$ \\
\hline$f_{23} / 10^{-4}$ & $0.5095-0.3396 i$ & & $2.342,41.69,42.08$ \\
\hline$f_{33} / 10^{-3}$ & $-0.4395-0.0514 i$ & $h[1,2,1]$ & $3.924,291.17,397.51$ \\
\hline$g_{12} / 10^{-3}$ & $-8.5548-3.4900 i$ & & $1580.89,2524.44$ \\
\hline$g_{13} / 10^{-3}$ & $-5.3631-18.5130 i$ & $I[3,1,10 / 3]$ & 0.82 \\
\hline$g_{23} / 10^{-2}$ & $61.1617+6.3854 i$ & $J[3,1,4 / 3]$ & $0.822,57.80,124.04$ \\
\hline$\lambda / 10^{-2}$ & $-0.0798+0.2049 i$ & & $124.04,1550.83$ \\
\hline$\eta$ & $-3.8375-2.4483 i$ & $K[3,1,8 / 3]$ & $54.56,2400.54$ \\
\hline$\rho$ & $4.3429+0.4901 i$ & $L[6,1,2 / 3]$ & $164.11,2693.32$ \\
\hline$k$ & $0.0163+0.0160 i$ & $M[6,1,8 / 3]$ & 2934.27 \\
\hline$\zeta$ & $2.1043-0.5613 i$ & $N[6,1,4 / 3]$ & 2549.57 \\
\hline $\bar{\zeta}$ & $2.0991-0.7985 i$ & $O[1,3,2]$ & 3774.62 \\
\hline$m / 10^{16} \mathrm{GeV}$ & 0.05463 & $P[3,3,2 / 3]$ & $521.70,2527.43$ \\
\hline$m_{\Theta} / 10^{16} \mathrm{GeV}$ & $-268.776 e^{-i \operatorname{Arg}(\lambda)}$ & $Q[8,3,0]$ & 0.396 \\
\hline$\gamma$ & 1.51935 & $R[8,1,0]$ & $0.45,1.23$ \\
\hline $\bar{\gamma}$ & 0.8968 & $S[1,3,0]$ & 1.3123 \\
\hline$x$ & $0.5241+1.1792 i$ & $t[3,1,2 / 3]$ & $1.36,28.99,95.99,300.01$ \\
\hline$\Delta_{X}$ & 2.15 & & $1166.70,1359.73,5170.19$ \\
\hline$\Delta_{G}$ & -17.689 & $U[3,3,4 / 3]$ & 0.996 \\
\hline$\Delta \alpha_{3}\left(M_{Z}\right)$ & -0.007 & $V[1,2,3]$ & 1.066 \\
\hline$\left\{M^{\nu^{c}} / 10^{11} \mathrm{GeV}\right\}$ & $0.0014,189.78,746.17$ & $W[6,3,2 / 3]$ & 1288.01 \\
\hline$\left\{M_{I I}^{\nu} / 10^{-11} \mathrm{eV}\right\}$ & $0.0004,50.34,197.92$ & $X[3,2,5 / 3]$ & $0.425,158.136,158.136$ \\
\hline$M_{\nu}(\mathrm{meV})$ & $1.0169,7.42,42.31$ & $Y[6,2,1 / 3]$ & 0.50 \\
\hline$\{$ Evals $[\mathrm{f}]\} / 10^{-5} \mathrm{eV}$ & $0.00008,11.530,45.33$ & $Z[8,1,2]$ & 1.21 \\
\hline Soft parameters & $\mathrm{m}_{\frac{1}{2}}=-558.176$ & $\mathrm{~m}_{0}=5739.456$ & $\overline{\mathrm{A}_{0}}=-2.8914 \times 10^{5}$ \\
\hline at $M_{X}^{0}$ & $\mu \stackrel{2}{=} 2.3744 \times 10^{5}$ & $\mathrm{~B}=-3.9561 \times 10^{10}$ & $\tan \beta=52.0000$ \\
\hline & $\mathrm{M}_{\overline{\mathrm{H}}}^{2}=-3.6652 \times 10^{10}$ & $\mathrm{M}_{\mathrm{H}}^{2}=-3.5552 \times 10^{10}$ & $\mathrm{R}_{\underline{\underline{b} \tau}}=1.0655$ \\
\hline $\operatorname{Max}\left(\left|L_{A B C D}\right|,\left|R_{A B C D}\right|\right)$ & $1.1270 \times 10^{-17} \mathrm{GeV}^{-1}$ & & \\
\hline
\end{tabular}

Table 9: Example 2: Column 1 contains values of the NMSGUT-SUGRY-NUHM parameters at $M_{X}$ derived from an accurate fit to all 18 fermion data and compatible with RG constraints. Unification parameters and mass spectrum of superheavy and superlight fields are also given. The values of $\mu\left(M_{X}^{0}\right), B\left(M_{X}^{0}\right)$ are determined by RG evolution from $M_{Z}$ to $M_{X}^{0}$ of the values determined by the EWRSB conditions. 


\begin{tabular}{|c|c|c|c|c|}
\hline & & & & \\
Parameter & Target $=\bar{O}_{i}$ & Uncert. $=\delta_{i}$ & Achieved $=O_{i}$ & Pull $=\left(O_{i}-\bar{O}_{i}\right) / \delta_{i}$ \\
\hline$y_{u} / 10^{-6}$ & 2.056309 & 0.785510 & 2.057005 & 0.000886 \\
$y_{c} / 10^{-3}$ & 1.002379 & 0.165393 & 1.003313 & 0.005643 \\
$y_{t}$ & 0.383757 & 0.015350 & 0.383780 & 0.001494 \\
$y_{d} / 10^{-5}$ & 6.031134 & 3.516151 & 5.966872 & -0.018276 \\
$y_{s} / 10^{-3}$ & 1.142585 & 0.539300 & 1.164037 & 0.039777 \\
$y_{b}$ & 0.601133 & 0.311988 & 0.602353 & 0.003909 \\
$y_{e} / 10^{-4}$ & 1.384241 & 0.207636 & 1.382867 & -0.006614 \\
$y_{\mu} / 10^{-2}$ & 2.795186 & 0.419278 & 2.795532 & 0.000827 \\
$y_{\tau}$ & 0.634068 & 0.120473 & 0.630898 & -0.026319 \\
$\sin _{12}^{q} \theta_{12}^{q}$ & 0.2210 & 0.001600 & 0.2210 & 0.0008 \\
$\sin \theta_{13}^{q} / 10^{-4}$ & 28.2913 & 5.000000 & 28.2975 & 0.0012 \\
$\sin \theta_{23}^{q} / 10^{-3}$ & 33.2889 & 1.300000 & 33.2836 & -0.0041 \\
$\delta^{q}$ & 60.0208 & 14.000000 & 60.1344 & 0.0081 \\
$\left(m_{12}^{2}\right) / 10^{-5}(e V)^{2}$ & 5.4009 & 0.572498 & 5.4011 & 0.0002 \\
$\left(m_{23}^{2}\right) / 10^{-3}(e V)^{2}$ & 1.7352 & 0.347036 & 1.7351 & -0.0003 \\
$\sin ^{2} \theta_{12}^{L}$ & 0.2925 & 0.058501 & 0.2925 & 0.0002 \\
$\sin ^{2} \theta_{23}^{L}$ & 0.4522 & 0.135654 & 0.4523 & 0.0012 \\
$\sin ^{2} \theta_{13}^{L}$ & 0.0244 & 0.019000 & 0.0243 & -0.0015 \\
\hline$\alpha_{1}$ & $0.4356+0.0000 i$ & $\bar{\alpha}_{1}$ & $0.6832+0.0000 i$ & \\
$\alpha_{2}$ & $0.0152-0.0348 i$ & $\bar{\alpha}_{2}$ & $0.0294-0.0607 i$ & \\
$\alpha_{3}$ & $0.0074-0.0199 i$ & $\bar{\alpha}_{3}$ & $0.0079-0.0331 i$ & \\
$\alpha_{4}$ & $-0.6267-0.6436 i$ & $\bar{\alpha}_{4}$ & $-0.5709-0.4473 i$ & \\
$\alpha_{5}$ & $0.0078-0.0160 i$ & $\bar{\alpha}_{5}$ & $-0.0265+0.0002 i$ & \\
$\alpha_{6}$ & $-0.0303-0.0112 i$ & $\bar{\alpha}_{6}$ & $-0.0039+0.0296 i$ & \\
\hline
\end{tabular}

Table 10: Example 2 :with $\chi_{X}=\sqrt{\sum_{i=1}^{17}\left(O_{i}-\bar{O}_{i}\right)^{2} / \delta_{i}^{2}}=0.0528$. Target values, at $M_{X}^{0}$ of the fermion Yukawa couplings and mixing parameters, together with the estimated uncertainties, achieved values and pulls. The eigenvalues of the wavefunction renormalization increment matrices $\Delta_{i}$ for fermion lines and the factors for Higgs lines are given, assuming the external Higgs is 10-plet dominated. The Higgs fractions $\alpha_{i}, \bar{\alpha}_{i}$ which control the MSSM fermion Yukawa couplings are also given. Notice the dominance of the first components $\alpha_{1}, \overline{\alpha_{1}}$ consistently with the assumption made. Right-handed neutrino threshold effects have been ignored. We have truncated numbers for display although all calculations are done at double precision. 


\begin{tabular}{|c|c|c|c|}
\hline Parameter & $S M\left(M_{Z}\right)$ & $m^{G U T}\left(M_{Z}\right)$ & $m^{M S S M}=(m+\Delta m)^{G U T}\left(M_{Z}\right)$ \\
\hline$m_{d} / 10^{-3}$ & 2.90000 & 0.47862 & 2.85870 \\
$m_{s} / 10^{-3}$ & 55.00000 & 9.33699 & 55.82819 \\
$m_{b}$ & 2.90000 & 3.19078 & 2.90012 \\
$m_{e} / 10^{-3}$ & 0.48657 & 0.46999 & 0.48432 \\
$m_{\mu}$ & 0.10272 & 0.09496 & 0.10258 \\
$m_{\tau}$ & 1.74624 & 1.73389 & 1.73348 \\
$m_{u} / 10^{-3}$ & 1.27000 & 1.08656 & 1.27132 \\
$m_{c}$ & 0.61900 & 0.52997 & 0.62010 \\
$m_{t}$ & 172.50000 & 146.48712 & 172.21615 \\
\hline
\end{tabular}

Table 11: Example 2: Values of standard model fermion masses in $\mathrm{GeV}$ at $M_{Z}$ compared with the masses obtained from values of GUT derived Yukawa couplings run down from $M_{X}^{0}$ to $M_{Z}$ both before and after threshold corrections. Fit with $\chi_{Z}=\sqrt{\sum_{i=1}^{9}\left(m_{i}^{M S S M}-m_{i}^{S M}\right)^{2} /\left(m_{i}^{M S S M}\right)^{2}}=$ 0.0227 .

\begin{tabular}{|c|c|c|c|}
\hline Parameter & Value & Parameter & Value \\
\hline$M_{1}$ & 198.83 & $M_{\tilde{u}_{1}}$ & 7246.47 \\
$M_{2}$ & 621.41 & $M_{\tilde{u}_{2}}$ & 7242.44 \\
$M_{3}$ & 592.21 & $M_{\tilde{u}_{3}}$ & 30148.11 \\
$M_{\tilde{\bar{l}}_{1}}$ & 2895.27 & $A_{11}^{0(l)}$ & -167770.16 \\
$M_{\tilde{\bar{l}}_{2}}$ & 219.15 & $A_{22}^{0(l)}$ & -167544.51 \\
$M_{\tilde{\bar{l}}_{3}}$ & 31721.05 & $A_{33}^{0(l)}$ & -92942.20 \\
$M_{\tilde{L}_{1}}$ & 11211.89 & $A_{11}^{0(u)}$ & -212183.50 \\
$M_{\tilde{L}_{2}}$ & 11025.49 & $A_{22}^{0(u)}$ & -212182.25 \\
$M_{\tilde{L}_{3}}$ & 25383.06 & $A_{33}^{0(u)}$ & -106623.25 \\
$M_{\tilde{\bar{d}}_{1}}$ & 2280.17 & $A_{11}^{0(d)}$ & -167689.77 \\
$M_{\tilde{\bar{d}}_{2}}$ & 2275.99 & $A_{22}^{0(d)}$ & -167688.64 \\
$M_{\tilde{\bar{d}}_{3}}$ & 72463.40 & $A_{33}^{0(d)}$ & -49795.07 \\
$M_{\tilde{Q}_{1}}$ & 8854.12 & $\tan \beta$ & 52.00 \\
$M_{\tilde{Q}_{2}}$ & 8851.95 & $\mu\left(M_{Z}\right)$ & 174787.15 \\
$M_{\tilde{Q}_{3}}$ & 55736.03 & $B\left(M_{Z}\right)$ & $5.4479 \times 10^{9}$ \\
$M_{\tilde{H}^{2}}^{2}$ & $-2.8079 \times 10^{10}$ & $M_{H}^{2}$ & $-3.4167 \times 10^{10}$ \\
\hline
\end{tabular}

Table 12: Example 2: Values $(\mathrm{GeV})$ in of the soft Susy parameters at $M_{Z}$ (evolved from the soft SUGRY-NUHM parameters at $M_{X}^{0}$ ). The values of soft Susy parameters at $M_{Z}$ determine the Susy threshold corrections to the fermion Yukawas. The matching of run down fermion Yukawas in the MSSM to the SM parameters determines soft SUGRY parameters at $M_{X}$. Note the heavier third sgeneration. The values of $\mu\left(M_{Z}\right)$ and the corresponding soft Susy parameter $B\left(M_{Z}\right)=m_{A}^{2} \sin 2 \beta / 2$ are determined by imposing electroweak symmetry breaking conditions. $m_{A}$ is the mass of the CP odd scalar in the in the Doublet Higgs. The sign of $\mu$ is assumed positive. 


\begin{tabular}{|c|c|}
\hline Field & Mass $(\mathrm{GeV})$ \\
\hline$M_{\tilde{G}}$ & 592.21 \\
$M_{\chi^{ \pm}}$ & $621.41,174787.19$ \\
$M_{\chi^{0}}$ & $198.83,621.41,174787.18,174787.18$ \\
$M_{\tilde{\nu}}$ & $11211.692,11025.294,25382.974$ \\
$M_{\tilde{e}}$ & $2895.63,11212.00,208.97,11025.89,25369.23,31732.18$ \\
$M_{\tilde{u}}$ & $7246.37,8853.95,7242.31,8851.81,30146.48,55737.26$ \\
$M_{\tilde{d}}$ & $2280.32,8854.33,2276.11,8852.17,55732.48,72466.16$ \\
$M_{A}$ & 532347.51 \\
$M_{H^{ \pm}}$ & 532347.52 \\
$M_{H^{0}}$ & 532347.50 \\
$M_{h^{0}}$ & 124.53 \\
\hline
\end{tabular}

Table 13: Example 2: Spectra of supersymmetric partners calculated ignoring generation mixing effects. Inclusion of such effects changes the spectra only marginally. Due to the large values of $\mu, B, A_{0}$. The LSP and light chargino are essentially pure Bino and Wino $\left(\tilde{W}_{ \pm}\right)$. The light gauginos and light Higgs $h^{0}$, are accompanied by a light smuon and sometimes selectron. The rest of the sfermions have multi-TeV masses. The mini-split supersymmetry spectrum and large $\mu, A_{0}$ parameters help avoid problems with FCNC and CCB/UFB instability [38,71]. The sfermion masses are ordered by generation not magnitude. This is useful in understanding the spectrum calculated including generation mixing effects

\begin{tabular}{|c|c|}
\hline Field & Mass $(\mathrm{GeV})$ \\
\hline$M_{\tilde{G}}$ & 586.63 \\
$M_{\chi^{ \pm}}$ & $620.15,175052.83$ \\
$M_{\chi^{0}}$ & $198.46,620.15,175052.81,175052.81$ \\
$M_{\tilde{\nu}}$ & $11020.47,11206.92,25306.768$ \\
$M_{\tilde{e}}$ & $301.27,2903.91,11021.07,11207.23,25292.91,31613.59$ \\
$M_{\tilde{u}}$ & $7268.78,7272.81,8837.25,8874.19,29777.84,55531.91$ \\
$M_{\tilde{d}}$ & $2371.66,2375.70,8837.65,8874.57,55527.11,72292.12$ \\
$M_{A}$ & 534359.68 \\
$M_{H^{ \pm}}$ & 534359.69 \\
$M_{H^{0}}$ & 534359.67 \\
$M_{h^{0}}$ & 123.59 \\
\hline
\end{tabular}

Table 14: Example 2: Spectra of supersymmetric partners calculated including generation mixing effects. Inclusion of such effects changes the spectra only marginally. Due to the large values of $\mu, B, A_{0}$. The LSP and light chargino are essentially pure Bino and Wino $\left(\tilde{W}_{ \pm}\right)$. Note that the ordering of the eigenvalues in this table follows their magnitudes, comparison comparison with the previous table is necessary to identify the sfermions 


\section{References}

[1] C. S. Aulakh, K. Benakli and G. Senjanovic, Phys. Rev. Lett. 79, 2188 (1997) [arXiv:hepph/9703434]; C. S. Aulakh, A. Melfo and G. Senjanovic, Phys. Rev. D 57 (1998) 4174 [arXiv:hep-ph/9707256]; C. S. Aulakh, A. Melfo, A. Rasin and G. Senjanovic, Phys. Lett. B 459, 557 (1999) [arXiv:hep-ph/9902409].

[2] C. S. Aulakh, B. Bajc, A. Melfo, A. Rasin and G. Senjanovic, Nucl. Phys. B 597, 89 (2001) [arXiv:hep-ph/0004031].

[3] C.S.Aulakh and A. Girdhar, hep-ph/0204097; v2 August 2003; v4, 9 February, 2004; Int. J. Mod. Phys. A 20, 865 (2005)

[4] B. Bajc, A. Melfo, G. Senjanovic and F. Vissani, Phys. Rev. D 70, 035007 (2004) [arXiv:hep$\mathrm{ph} / 0402122]$.

[5] B. Bajc, A. Melfo, G. Senjanovic and F. Vissani, AIP Conf. Proc. 805, 152 (2006) [AIP Conf. Proc. 805, 326 (2006)] [arXiv:hep-ph/0511352].

[6] C. S. Aulakh and A. Girdhar, Nucl. Phys. B 711 (2005) 275 [arXiv:hep-ph/0405074].

[7] T. Fukuyama, A. Ilakovac, T. Kikuchi, S. Meljanac and N. Okada, Eur. Phys. J. C 42, 191 (2005) arXiv:hep-ph/0401213v1.,v2.

[8] K. Y. Oda, E. Takasugi, M. Tanaka and M. Yoshimura, Phys. Rev. D 59, 055001 (1999) [arXiv:hep-ph/9808241]; K. Matsuda, Y. Koide, T. Fukuyama and H. Nishiura, Phys. Rev. D 65, 033008 (2002) [Erratum-ibid. D 65, 079904 (2002)] [arXiv:hep-ph/0108202] ; K. Matsuda, Y. Koide and T. Fukuyama, Phys. Rev. D 64, 053015 (2001) [arXiv:hep-ph/0010026]. N. Oshimo, ; Phys. Rev. D 66, 095010 (2002) [arXiv:hep-ph/0206239]; N. Oshimo, Nucl. Phys. B 668, 258 (2003) [arXiv:hep-ph/0305166]; B. Bajc, G. Senjanovic and F. Vissani, Phys. Rev. Lett. 90 (2003) 051802 [arXiv:hep-ph/0210207]; H. S. Goh, R. N. Mohapatra and S. P. Ng, Phys. Lett. B 570, 215 (2003) [arXiv:hep-ph/0303055]. H. S. Goh, R. N. Mohapatra and S. P. Ng, Phys. Rev. D 68, 115008 (2003) [arXiv:hep-ph/0308197]. H. S. Goh, R. N. Mohapatra and S. Nasri, Phys. Rev. D 70 (2004) 075022 [arXiv:hep-ph/0408139]; B. Bajc, G. Senjanovic and F. Vissani, Phys. Rev. D 70, 093002 (2004) [arXiv:hep-ph/0402140]; B. Bajc, G. Senjanovic and F. Vissani, arXiv:hep-ph/0110310;K. S. Babu and C. Macesanu, Phys. Rev. D 72, 115003 (2005) [arXiv:hep-ph/0505200].

[9] P. Minkowski, Phys. Lett. B67,110(1977); M. Gell-Mann, P. Ramond and R. Slansky, in Supergravity, eds. P. van Niewenhuizen and D.Z. Freedman (North Holland 1979); T. Yanagida, in Proceedings of Workshop on Unified Theory and Baryon number in the Universe, eds. O. Sawada and A. Sugamoto (KEK 1979); R.N. Mohapatra and G. Senjanović, Phys. Rev. Lett. 44, 912 (1980); R.N. Mohapatra and G. Senjanović, Phys. Rev. D23,165 (1981); G. Lazarides, Q. Shafi and C. Wetterich, Nucl. Phys. B181, 287 (1981).

[10] C.S. Aulakh and R.N. Mohapatra, CCNY-HEP-82-4 April 1982, CCNY-HEP-82-4-REV, Jun 1982 , Phys. Rev. D28, 217 (1983).

[11] T.E. Clark, T.K.Kuo, and N.Nakagawa, Phys. lett. 115B, 26(1982).

[12] C. S. Aulakh, B. Bajc, A. Melfo, G. Senjanovic and F. Vissani, Phys. Lett. B 588, 196 (2004) [arXiv:hep-ph/0306242]. 
[13] R. N. Mohapatra, Phys. Rev. D 34, 3457 (1986).; A. Font, L. E. Ibanez and F. Quevedo, Phys. Lett. B 228, 79 (1989); S. P. Martin, Phys. Rev. D 46, 2769 (1992) [arXiv:hep-ph/9207218]; D. G. Lee and R. N. Mohapatra, Phys. Rev. D 51, 1353 (1995) [arXiv:hep-ph/9406328].

[14] K.S.Babu and R.N.Mohapatra, Phys. Rev. Lett. 70(1993)2845.

[15] C. S. Aulakh, From germ to bloom, arXiv:hep-ph/0506291.

[16] C. S. Aulakh and S. K. Garg, Nucl. Phys. B 757, 47 (2006) [arXiv:hep-ph/0512224].

[17] S. Bertolini, T. Schwetz and M. Malinsky, Phys. Rev. D 73 (2006) 115012 [arXiv:hep$\mathrm{ph} / 0605006]$.

[18] S. Bertolini, M. Frigerio and M. Malinsky, Phys. Rev. D 70, 095002 (2004) [arXiv:hepph/0406117]; S. Bertolini and M. Malinsky, arXiv:hep-ph/0504241 ;

[19] B. Dutta, Y. Mimura and R. N. Mohapatra, Phys. Lett. B 603 (2004) 35 [arXiv:hepph/0406262]; B. Dutta, Y. Mimura and R. N. Mohapatra, Phys. Rev. Lett. 94 (2005) 091804 [arXiv:hep-ph/0412105]; B. Dutta, Y. Mimura and R. N. Mohapatra, Phys. Rev. D 72 (2005) 075009 [arXiv:hep-ph/0507319];

[20] L. Lavoura, H. Kühböck and W. Grimus, Nucl. Phys. B 754 (2006) 1 [arXiv:hep-ph/0603259].

[21] W. Grimus and Kühböck, Phys. Lett. B 643, 182 (2006) [arXiv:hep-ph/0607197].

[22] W. Grimus and Kühböck, Eur. Phys. J. C51 (2007) 721-729. [hep-ph/0612132].

[23] C. S. Aulakh, Phys. Lett. B 661, 196 (2008) [arXiv:0710.3945 [hep-ph]].

[24] C. S. Aulakh and S. K. Garg, Mod. Phys. Lett. A 24 (2009) 1711 [arXiv:0710.4018 [hep-ph]].

[25] See earlier arXiv versions of this work : C. S. Aulakh and S. K. Garg, The new minimal supersymmetric GUT, arXiv:hep-ph/0612021v1; arXiv:hep-ph/0612021v2.

[26] H. Georgi and C. Jarlskog, Phys. Lett. B 86 (1979) 297.

[27] L. J. Hall, R. Rattazzi and U. Sarid, Phys. Rev. D 50 (1994) 7048 [arXiv:hep-ph/9306309]; R. Hempfling, Phys. Rev. D49(1994)6168.

[28] M. S. Carena, M. Olechowski, S. Pokorski and C. E. M. Wagner, Nucl. Phys. B 426, 269 (1994) [arXiv:hep-ph/9402253]; M. S. Carena, D. Garcia, U. Nierste and C. E. M. Wagner, Nucl. Phys. B 577, 88 (2000) [arXiv:hep-ph/9912516].

[29] G. Ross and M. Serna, arXiv:0704.1248 [hep-ph].

[30] S. Antusch and M. Spinrath, arXiv:0804.0717 [hep-ph].

[31] S. P. Martin and M. T. Vaughn, Phys. Rev. D 50 (1994) 2282 [arXiv:hep-ph/9311340]; S. P. Martin, Phys. Rev. D 66 (2002) 096001 [arXiv:hep-ph/0206136].

[32] K. S. Babu, C. N. Leung and J. T. Pantaleone, Phys. Lett. B 319, 191 (1993) [arXiv:hep$\mathrm{ph} / 9309223]$.

[33] S. Antusch, M. Drees, J. Kersten, M. Lindner and M. Ratz, Phys. Lett. B 519, 238 (2001) [arXiv:hep-ph/0108005]; S. Antusch, M. Drees, J. Kersten, M. Lindner and M. Ratz, Phys. Lett. B 525, 130 (2002) [arXiv:hep-ph/0110366]. 
[34] Lepton-Photon,Mumbai (2011): http://www.tifr.res.in/ lp11/program.html.

[35] H. Murayama and A. Pierce, Phys. Rev. D 65, 055009 (2002), [arXiv:hep-ph/0108104].

[36] B. Bajc, P. Fileviez Perez and G. Senjanovic, Phys. Rev. D 66 (2002) 075005 [arXiv:hep$\mathrm{ph} / 0204311]$.

[37] C. S. Aulakh and S. K. Garg, Nmsgut II: Fermion Fits and Soft Spectra, arXiv:0807.0917v2 [hep-ph].

[38] C. S. Aulakh, Grand Unification upended, arXiv:1107.2963 [hep-ph].

[39] H. Arason, D. J. Castano, B. Keszthelyi, S. Mikaelian, E. J. Piard, P. Ramond and B. D. Wright, Phys. Rev. D 46 (1992) 3945; H. Arason, D. J. Castano, E. J. Piard and P. Ramond, [arXiv:hep-ph/9204225].

[40] V. Lucas and S. Raby, Phys. Rev. D 55 (1997) 6986 [arXiv:hep-ph/9610293].

[41] T. Goto and T. Nihei, Phys. Rev. D 59, 115009 (1999) [arXiv:hep-ph/9808255].

[42] A. Melfo and G. Senjanovic, arXiv:hep-ph/0511011.

[43] C. S. Aulakh, Fermion mass hierarchy in the Nu MSGUT. I: The real core, arXiv:hep$\mathrm{ph} / 0602132$

[44] C. S. Aulakh, MSGUT Reborn? arXiv:hep-ph/0607252

[45] S. Weinberg, Phys. Rev. Lett. 43 (1979) 1566.

[46] L.J.Hall, Nucl. Phys. B178,75(1981).

[47] W. Siegel, Phys. Lett. 84B, 193, (1979); D. M. Capper, D. R. T. Jones and P. van Nieuwenhuizen, Nucl. Phys. B167, 479, (1980); S. P. Martin and M. T. Vaughn, Phys. Lett. 318B, 331, (1993).

[48] K. Nakamura et al. (Particle Data Group), J. Phys. G 37, 075021 (2010)

[49] P. Langacker and N. Polonsky, Phys. Rev. D 47, 4028 (1993) [arXiv:hep-ph/9210235].

[50] P. Langacker and N. Polonsky, Phys. Rev. D 52, 3081 (1995) [arXiv:hep-ph/9503214].

[51] C. S. Aulakh, Phys. Rev. D 72, 051702 (2005), arXiv:hep-ph/0501025.

[52] C. S. Aulakh, arXiv:0807.1792 [hep-ph].

[53] D. J. Castano, E. J. Piard and P. Ramond, "Renormalization group study of the Standard Model and its extensions. 2. The Minimal supersymmetric Standard Model," Phys. Rev. D 49, 4882 (1994) [arXiv:hep-ph/9308335].

[54] V.V. Dixit and M. Sher, Phys. Rev. D40,3765(1989).

[55] Z. Z. Xing, H. Zhang and S. Zhou, arXiv:0712.1419 [hep-ph].

[56] A. Strumia and F. Vissani, arXiv:hep-ph/0606054.

[57] T2K collaboration, Indication of Electron Neutrino Appearance from an Acceleratorproduced Off-axis Muon Neutrino Beam, arXiv:1106.2822. 
[58] E. K. Akhmedov, Z. G. Berezhiani, G. Senjanovic and Z. j. Tao, Phys. Rev. D 47 (1993) 3245 [arXiv:hep-ph/9208230].

[59] Numerical Recipes in Fortran 90, Second Edition(1996), Cambridge Univ. Press, W.H.Press, S.A.Teukolsky, W.T.Vetterling, and B.P.Flannery.

[60] D. M. Pierce, J. A. Bagger, K. T. Matchev and R. j. Zhang, Nucl. Phys. B 491, 3 (1997) [arXiv:hep-ph/9606211].

[61] A. Freitas, E. Gasser and U. Haisch, Phys. Rev. D 76 (2007) 014016 [arXiv:hep-ph/0702267].

[62] S. F. King and M. Oliveira, Phys. Rev. D 63 (2001) 015010 [arXiv:hep-ph/0008183].

[63] Theory and Phenomenology of Sparticles, M.Drees, R.Godbole, and P.Roy,World Scientific Publishing Co. Pte. Ltd.(Singapore) 2004.

[64] W. Porod, Comput. Phys. Commun. 153 (2003) 275 [arXiv:hep-ph/0301101] ; W. Porod and F. Staub arXiv:1104.1573.

[65] J.Ellis, G.Ridolfi, F.Zwirner, Phys. Lett. B262(1991) 477.

[66] Y.Hayato et al. ,[Super-Kamiokande collaboration], Phys. Rev. Lett. 83,1529(1999); K.Kobayashi et al. ,[Super-Kamiokande collaboration][hep-ex/0502026].

[67] C. S. Aulakh, "Truly minimal unification: Asymptotically strong panacea?," arXiv:hep$\mathrm{ph} / 0207150$.

[68] C. S. Aulakh, "Taming asymptotic strength," arXiv:hep-ph/0210337.

[69] F. David, "A Comment On Induced Gravity," Phys. Lett. B 138, 383 (1984); F. David and A. Strominger, "On The Calculability Of Newton's Constant And The Renormalizability Of Scale Invariant Quantum Gravity," Phys. Lett. B 143 (1984) 125.

[70] N. Seiberg, Phys. Lett. B318, 469(1993); N. Seiberg, Phys. Rev.D49, 6857 (1994). For reviews and complete references see : K.Intriligator, N.Seiberg, Proc. of ICTP Summer School, 1995, Nucl. Phys. Proc. Suppl. 45BC,1(1996), hep-th/9509066; M.Shifman,Lectures at ICTP Summer School, 1996, Prog. Part. Nucl. Phys. 39,1(1997).

[71] A. Kusenko, P. Langacker and G. Segre, Phys. Rev. D 54 (1996) 5824 [arXiv:hep$\mathrm{ph} / 9602414]$. 